\title{
Ueber diejenigen Curven, deren Coordinaten sich als elliptische Functionen eines Parameters darstellen lassen.
}

(Von Herrn A. Clebsch zu Giessen.)

Der vorliegende Aufsatz behandelt die Theorie desjenigen Geschlechts ebener Curven, für welche in dem nach Riemann von mir festgehaltenen Sinne $p=1$, welche also mit Hülfe nichtlinearer Projection in Curven dritter Ordnung übergeführt werden können. Diese Curven besitzen, wenn ihre Ordnung $n$ ist, $\frac{n . n-3}{2}$ Doppel - und Rückkehrpunkte; ihre Natur hängt wesentlich von einer einzigen Constante $a b$, dem Modul der entsprechenden elliptischen Functionen, und diese characteristische Constante hat die einfache geometrische Bedeutung eines bestimmten unveränderlichen Doppelverhältnisses, ähnlich wie bei den Curven dritter Ordnung der Modul dies Doppelverhältniss der von einem Punkt der Curven an sie gezogenen Tangenten angiebt.

Entspricht so jedem Punkt der Curve ein bestimmter Werth eines elliptischen Integrals und umgekehrt, so giebt das Abel'sche Theorem für die zwischen den Schnittpunkten der Curve mit einer andern algebraischen Curve zu erfüllenden Bedingungen sehr einfache Relationen zwischen den entprechenden Integralen erster und zwischen gewissen Integralen dritter Gattung. Die Aufgabe, aus einigen Punkten des Schnittpunktsystems die übrigen zu finden, führt sodann zu einem Umkehrungsprobleme, dessen speciellsten Fall Hr. Rosenhain in seiner Preisschrift behandelt hat. Das allgemeine Problem, dessen Lösung hier gegeben wird, ist folgendes: Die Summe von $m$ Integralen erster Gattung, und $m-1$ Summen von je $m$ gleichartigen Integralen dritter Gattung sind gegeben; man soll eine Gleichung $\boldsymbol{m}^{\text {ten }}$ Grades angeben, deren Wurzeln die $m$ in jenen Integralen auftretenden oberen Grenzen sind.

Als Anwendung der allgemeinen Theorie habe ich denjenigen Fall behandelt, welcher nächst den Curven dritter Ordnung der einfachste ist, und welcher zugleich bei vorhandenen Doppelpunkten das Wesen der hier anzustellenden Untersuchungen vollkommen erkennen lässt, nämlich die Curven vierter Ordnung mit zwei Doppelpunkten. 
\$. 1.

Characterisirung der zu betrachtenden Curven. Ihre Coordinaten werden mit Hülfe eines Systems von Curven $(n-2)^{\text {ter }}$ Ordnung durch einen Parameter ausgedrückt.

Diejenigen algebraischen Curven $n^{\text {ter }}$ Ordnung, welche es gestatten, ihre Coordinaten als rationale Functionen von $\sin \operatorname{am} u, \cos \operatorname{am} u, \Delta$ am $u$ auszudrücken, wobei $u$ die Stelle eines veränderlichen Parameters versieht, werden dadurch characterisirt, dass sie $\frac{n . n-3}{2}$ Doppel- resp. Rückkehrpunkte besitzen. Die wirkliche Darstellung der Coordinaten in jener Form kann man folgendermassen ausführen.

Die $\frac{n . n-3}{2}$ Doppelpunkte (Rückkehrpunkte als specielle Fälle immer mit einbegriffen) bestimmen zusammen eine einzige Curve $(n-3)^{\text {ter }}$ Ordnung, welche sich durch dieselben legen lässt. Will man aber Curven $n-2^{\text {ter }}$ Ordnung hindurchlegen, so kann man auf der gegebenen Curve noch $(n-2)$ weitere Punkte beliebig annehmen. Diese mit den Doppelpunkten zusammen bestimmen einen Büschel von Curven $(n-2)^{\text {ter }}$ Ordnung, deren jede die gegebene noch in zwei andern Punkten schneidet. Sei $f=0$ die Gleichung der gegebenen Curve, $u+\lambda v=0$ die Gleichung des Büschels, und legen wir einen Büschel von Geraden $a+\mu b=0$ mit beliebigem Scheitel, deren Strahlen, nach den Durchschnitten von $f=0$ mit $u+\lambda v=0$ gerichtet sind. Eliminiren wir nun aus den Gleichungen

$$
\text { (1.) } f=0, \quad u+\lambda v=0, \quad a+\mu b=0
$$

die Coordinaten, so erhalten wir eine Resultante $\Omega(\lambda, \mu)=0$, und zugleich die, Vèrhältnisse der Coordinaten als rationale Functionen von $\lambda, \mu$. Untersuchen wir diese Ausdrücke und die Resultante $\Omega$ genauer.

Wenn wir, ohne auf die gegenseitigen Beziehungen der Coefficienten von $f, u, v$ zu achten, diese Bildungen vornehmen, so gelangen wir zu einer Resultante $\Omega$, welche vom Grade $n--2$ für die Coefficienten von $f$, vom Grade $n$ für die von $u+\lambda \dot{v}$, und vom Grade $n . n-2$ für die von $a+\mu b$ ist. Betrachten wir die Gleichung $\Omega=0$ als Gleichung für $\lambda$, so giebt sie die $n$ Parameter, deren entsprechende Curven durch je einen der $n$ Schnittpunkte von $f$ mit einem Strahl $a+\mu b$ hindurchgehen. Setzen wir dagegen an die Stelle der Coefficienten von $a+\mu b$ beliebige Liniencoordinaten, und betrachten diese als Veränderliche, so stellt $\Omega$ offenbar das Product von $n . n-2$ linearen Factoren, also den Complex derjenigen $n . n-2$ Punkte dar, in denen $f$ yon der Curve $u+\lambda v$ getroffen wird. 
212 Clebsch, ebene Curven, deren Coord. elliptische Funct. eines Parameters sind.

Bezeichnen wir ferner durch $f_{i k h}, u_{i k h}, v_{i k h}$ die Coefficienten von $x_{1}^{i} x_{2}^{k} x_{3}^{h}$ in $f, u, v$, und setzen wir

$$
a=a_{1} x_{1}+a_{2} x_{2}+a_{3} x_{3}, \quad b=b_{1} x_{1}+b_{2} x_{2}+b_{3} x_{3},
$$

so werden nach bekannten Regeln (vgl. Salmon, Algebra der linearen Transformationen, übers. v. Fiedler, p. 74.) die Verhältnisse der den Gleichungen (1.) genügenden Coordinaten aus folgenden Formeln gefunden:

$$
\left\{\begin{array}{rlrl}
\rho x_{1}^{i} x_{2}^{k} x_{3}^{h} & =\frac{\partial \Omega}{\partial f_{i k h}}, & (i+k+h=n), \\
\sigma x_{1}^{i} x_{2}^{k} x_{3}^{h} & =\frac{\partial \Omega}{\partial u_{i k h}}, & (i+k+h=n-2), \\
\tau x_{i} & =\frac{\partial \Omega}{\partial a_{i}} .
\end{array}\right.
$$

\section{\$. 2.}

Untersuchung der Discriminante von $\boldsymbol{\Omega}=\mathbf{0}$.

Die Discriminante $D$ der Gleichung $\Omega$ in Bezug auf $\mu$ ist, da $\Omega$ selbst für $\mu$ vom Grade $n . n-2$ ist, vom Grade $2\left(n^{2}-2 n-1\right)$ für die Coefficienten von $\Omega$, also vom Grade $2 n\left(n^{2}-2 n-1\right)$ für $\lambda$, und vom Grade $2 n(n-2)\left(n^{2}-2 n-1\right)$ in Bezug auf die $a$ und $b$. Setzt man $D=0$, so entsteht eine Gleichung für $\lambda$, welche diejenigen Werthe liefert, für welche zwei Wurzeln $\mu$ einander gleich werden.

Das letztere aber kann auf doppelte Weise geschehen. Erstlich kann eine solche Curve $u+\lambda v=0$ die Curve $f$ berühren, und deswegen zwei Strahlen $a+\mu b$, welche nach dem Berührungspunkt gezogen werden, zusammenrücken. Oder zwei Schnittpunkte von $f$ mit $u+\lambda v$ liegen in einer Geraden mit dem Büschelscheitel, und diese Gerade wird deswegen Doppelstrahl. Ich werde zeigen, dass jeder Werth von $\lambda$, für den das letztere eintritt, eine Doppelwurzel der Gleichung $D=0$ ist.

Für einen solchen Werth von $\lambda$ und den zugehörigen von $\mu$ ist nämlich erstens, weil zwei Werthe von $\mu$ zusammenfallen, $\frac{\partial \Omega}{\partial \mu}=0$. Aber zugleich werden die Werthe der $x$ nothwendig unbestimmt, weil zwei Punkte $x$ gleichzeitig allen Bedingungen genũgen; es müssen also die rechten Theile von (2.) sämmtlich verschwinden, so dass auch

$$
\frac{\partial \boldsymbol{\Omega}}{\partial \lambda}=\boldsymbol{\Sigma} \boldsymbol{v}_{i k h} \frac{\partial \boldsymbol{\Omega}}{\partial u_{i k h}}=\mathbf{0} \text {. }
$$


Denken wir uns also $\Omega(\lambda, \mu)=0$ als Gleichung einer Curve, deren rechtwinklige Coordinaten $\lambda, \mu$ sind, so hat diese Curve bei dem in Rede stehenden Werthepaar $\lambda, \mu$ einen Doppelpunkt. Aber $D=0$, welches das Zusammenbestehen von $\Omega=0, \frac{\partial \Omega}{\partial \mu}=0$ angiebt, ist die Gleichung, welche die der Axe $\mu$ parallelen Tangenten der Curve liefert. Da nun ein Doppelpunkt stets zwei Tangenten absorbirt, so ist auch der fragliche Werth von $\lambda$ eine Doppelwurzel von $D=0$.

Untersuchen wir jetzt, welche Modificationen diese Verhältnisse erfahren, wenn man die zwischen den Coefficienten von $f, u$, stattfindenden Beziehungen berücksichtigt.

Die $n . n-2$ Punkte, deren Gleichungen $\Omega=0$ darstellte, wenn man darin die $a+\mu b$ durch veränderliche Liniencoordinaten ersetzte, sondern sich hier in die $\frac{n . n-3}{2}$ Doppelpunkte, welche immer zweimal zu rechnen sind, in die $n-2$ festen einfachen Punkte, welche auf $f=0$ gewählt wurden, und in nur zwei veränderliche Punkte. Die Gleichung $\Omega=0$, wenn darin die $a+\mu b$ durch Liniencoordinaten ersetzt sind, nimmt also die Form an

$$
\Omega_{1}^{2} \cdot \Omega_{2} \cdot \Omega_{0}=0,
$$

wo $\Omega_{1}=0$ das Product der Gleichungen aller Doppelpunkte, $\Omega_{2}=0$ das Product der Gleichungen aller festen einfachen Punkte, und endlich $\Omega_{1}=0$ das Product der Gleichungen der beiden beweglichen Punkte ist, um welche allein es sich handelt.

Der Factor $\Omega_{v}$ enthält $\lambda$, nicht so die andern Factoren, welche feste, d. h. von $\lambda$ unabhängige Punkte darstellen. Es ist also $\Omega_{0}$ von der Form

$$
\text { (3.) } \Omega_{0}=\Sigma \Sigma \omega_{i k} \gamma_{i} \gamma_{k},
$$

wobei die $\omega$ Functionen $n^{\text {ter }}$ Ordnung in $\lambda$, die $\gamma$ Liniencoordinaten darstellen; und da $\Omega_{0}$ das Product der Gleichungen zweier Punkte ist, so hat man nothwendig auch

$$
\text { (4.) } \quad W=\left|\begin{array}{lll}
\omega_{11} & \omega_{12} & \omega_{13} \\
\omega_{21} & \omega_{22} & \omega_{23} \\
\omega_{31} & \omega_{32} & \omega_{33}
\end{array}\right|=0 \text {. }
$$

Sind $W_{i k}$ die Unterdeterminanten der $W$, so ist nach bekannten Regeln

$$
\text { (5.) } \quad \Sigma \Sigma W_{i k} x_{i} x_{k}=L^{2}=0
$$

das Quadrat der Gleichung der Verbindungslinie der beiden beweglichen Punkte, 
214 Clebsch, ebene Curven, deren Coord. elliptische Funct. eines Parameters sind.

welche, indem $\lambda$ sich ändert, eine Curve von einer weiterhin zu bestimmenden Classe umhüllt.

Die Gleichung für $\mu$ wird, nach Absonderung des von $\Omega_{1}^{2} \cdot \Omega_{2}$ herrührenden, von $\lambda$ freien Factors, von der Form

$$
\text { (6.) } A+2 \mu C+\mu^{2} B=0 \text {, }
$$

und zwar entsteht die linke Seite dieser Gleichung, wenn man in (3.) $\gamma_{i}$ durch $a_{i}+\mu b_{i}$ ersetzt; es ist also

$$
\left\{\begin{array}{l}
A=\Sigma \Sigma \omega_{i k} a_{i} a_{k}, \\
B=\Sigma \Sigma \omega_{i k} b_{i} b_{k}, \\
C=\Sigma \Sigma \omega_{i k} a_{i} b_{k} .
\end{array}\right.
$$

Die Discriminante $A B-C^{2}$ ist vom Grade $2 n$ in $\lambda$, und hat die Form $P^{2} . Q$, wo $Q=0$ diejenigen Werthe von $\lambda$ giebt, deren entsprechende Curven $u+\lambda v=0$ die Curve $f=0$ berühren, während $P=0$ solche Curven $u+\lambda v=0$ liefert, bei denen die beweglichen Schnittpunkte mit dem Büschelscheitel $(a=0, b=0)$ in einer Geraden liegen. Die Grade von $P$ und $Q$ sollen weiterhin bestimmt werden. Die Auflösung der Gleichung (6.) giebt dann:

$$
\text { (8.) } \mu=\frac{-C+P \sqrt{-Q}}{B} \text {. }
$$

Die Discriminante lässt sich aber noch in einer andern bemerkenswerthen Form darstellen; nach bekannten Determinantensätzen hat man nämlich auch:

$$
\text { (9.) } \quad A B-C^{2}=P^{2} Q=\Sigma \Sigma W_{i k} p_{i} p_{k},
$$

wo $p_{i}, p_{k}$ die Coordinaten des Büschelscheitels $a, b$ sind, also:

$$
\text { (10.) }\left\{\begin{array}{l}
p_{1}=a_{2} b_{3}-b_{2} a_{3}, \\
p_{2}=a_{3} b_{1}-b_{3} a_{1}, \\
p_{3}=a_{1} b_{2}-b_{1} a_{2} .
\end{array}\right.
$$

Endlich liefert die letzte Gleichung (2.) die Coordinaten der beweglichen Schnittpunkte. Statt $\Omega$ kann man rechts $\Omega_{0}$ setzen, und es ist also:

$$
\rho x_{i}=\left(\omega_{1 i} a_{1}+\omega_{2 i} a_{2}+\omega_{3 i} a_{3}\right)+\mu\left(\omega_{1 i} b_{1}+\omega_{2 i} b_{2}+\omega_{3 i} b_{3}\right) \text {. }
$$

\$. 3.

Curven des Systems $(\boldsymbol{n}-2)^{\text {ter }}$ Ordnung, welche die Curve $\boldsymbol{n}^{\text {ter }}$ Ordnung berühren.

Ehe man nun diese Ausdrücke weiter transformiren kann, ist es nothwendig, die Natur der Functionen $P, Q$ genauer zu erforschen. Man findet die Wurzeln von $Q$ mit Hülfe folgender Betrachtung. 
Die Werthe von $\lambda$, für welche $Q$ verschwindet, sind dadurch characterisirt, dass die beiden beweglichen Schnittpunkte einander unendlich nahe rücken, dass also die Curven $f=0, u+\lambda v=0$ einander berühren. Bezeichnen wir durch angehängte Indices Differentialquotienten nach den $x$, so sind hierzu folgende Bedingungen nöthig:

$$
\begin{aligned}
& \rho f_{1}=u_{1}+\lambda v_{1}, \\
& \varrho f_{2}=u_{2}+\lambda v_{2}, \quad f=0 . \\
& \varrho f_{3}=u_{3}+\lambda v_{3} .
\end{aligned}
$$

Indem man aber $\rho$ eliminirt, erhält man die Gleichungen

$$
\left\{\Theta=\left|\begin{array}{ccc}
f_{1} & f_{2} & f_{3} \\
u_{1} & u_{2} & u_{3} \\
v_{1} & v_{2} & v_{3}
\end{array}\right|=0, \quad f=0,\right.
$$

deren Durchschnittspunkte diejenigen Punkte angeben, in welchen eine Curve des Büschels $u+\lambda v=0$ die Curve $f=0$ berührt. Aber eine genauere Betrachtung zeigt, dass von den Durchschnittspunkten von $\Theta=0, f=0$ einige der Frage fremd sind. Denn in der That schneiden diese Curven sich mehrfach in den festgelegten Punkten des Büschels; aber diese können nicht Wurzeln der Discriminante $P^{2} Q$ geben, weil, wenn auch in diesen Punkten eine Curve $u+\lambda v=0$ die Curve $f=0$ berühren kann, dennoch für sie nicht zwei Factoren von $\Omega_{0}$ einander gleich werden, sondern nur ein Factor von $\Omega_{0}$ einem Factor von $\Omega_{1}$ oder $\Omega_{2}$ gleich wird.

Die Natur der Curve $\Theta=0$ (nach Hrn. Cremona die Jacobische Curve des Systems $f, u, v$ genannt) ist mehrfach untersucht worden, und es ist namentlich der Satz bekannt, dass diese Curve durch etwaige gemeinsame Punkte der drei Curven $f, u, v$ hindurchgeht (Cremona, introd. ad una teor. geom. delle curve piane p. 73.). Ferner hat Hr. Hesse bewiesen (dieses Journal Bd. 41. p. 286), dass, wenn wie hier zwei Functionen von gleichem Grade sind, für einen Schnittpunkt aller drei Curven die Differentialquotienten von $\Theta$ sich wie die der Curve ungleichen Grades (hier $f$ ) verhalten, dass also in solchen Punkten die Jacobische Curve die dritte Curve berïhrt. Diesem kann man folgenden weitern Satz hinzufügen:

Sind $u=0, v=0$ von gleichem Grade, und gehen sie durch einen Doppelpunkt von $f=0$, so hat auch $\Theta$ in diesem Punkte einen Doppelpunkt, und zwar sind die Tangenten des Doppelpunkts für beide Curven dieselben. 
216 Clebsch, ebene Curven, deren Coord. elliptische Funct. eines Parameters sind.

Den Beweis dieses Satzes kann man ähnlich führen, wie Herr Hesse a. a. 0. seine Sätze bewiesen hat. Sind $c_{1}, c_{2}, c_{3}$ irgend welche Constanten, so ist immer identisch

$$
\Theta .\left(c_{1} x_{1}+c_{2} x_{2}+c_{3} x_{3}\right)=-\left|\begin{array}{cccc}
f_{1} & f_{2} & f_{3} & n f \\
u_{1} & u_{2} & u_{3} & m u \\
v_{1} & v_{2} & v_{3} & m v \\
c_{1} & c_{2} & c_{3} & 0
\end{array}\right|,
$$

wobei der Allgemeinheit wegen $m$ für $(n-2)$ gesetzt ist, da es nur darauf ankommt, dass $u, v$ den gleichen Grad $m$ besitzen. Da im Doppelpunkte die Ausdrücke $u, v, f, f_{1}, f_{2}, f_{3}$ gleichzeitig verschwinden, so sind auch die Determinänten

$$
\left|\begin{array}{lll}
f_{i 1} & f_{i 2} & f_{i 3} \\
u_{1} & u_{2} & u_{3} \\
v_{1} & v_{2} & v_{3}
\end{array}\right|
$$

gleich Null. Differentiirt man also (13.) zweimal, und zwar nach $x_{i}$ und $x_{k}$, und lässt man alles im Doppelpunkt verschwindende fort, so kommt:

$$
\begin{aligned}
& \left(c_{1} x_{1}+c_{2} x_{2}+c_{3} x_{3}\right) \Theta_{i k}=-\left|\begin{array}{cccc}
f_{1 i k} & f_{2 i k} & f_{3 i k} & n f_{i k} \\
u_{1} & u_{2} & u_{3} & 0 \\
v_{1} & v_{2} & v_{3} & 0 \\
c_{1} & c_{2} & c_{3} & 0
\end{array}\right| \\
& -\left|\begin{array}{cccc}
f_{1 i} & f_{2 i} & f_{3 i} & 0 \\
u_{1 k} & u_{2 k} & u_{3 k} & m u_{k} \\
v_{1} & v_{2} & v_{3} & 0 \\
c_{1} & c_{2} & c_{3} & 0
\end{array}\right|-\left|\begin{array}{cccc}
f_{1 k} & f_{2 k} & f_{3 k} & 0 \\
u_{1 i} & u_{2 i} & u_{3 i} & m u_{i} \\
v_{1} & v_{2} & v_{3} & 0 \\
c_{1} & c_{2} & c_{3} & 0
\end{array}\right|-\left|\begin{array}{cccc}
f_{1 i} & f_{2 i} & f_{3 i} & 0 \\
u_{1} & u_{2} & u_{3} & 0 \\
v_{1 k} & v_{2 k} & v_{3 k} & m v_{k} \\
c_{1} & c_{2} & c_{3} & 0
\end{array}\right|-\left|\begin{array}{cccc}
f_{1 k} & f_{2 k} & f_{3 k} & 0 \\
u_{1} & u_{2} & u_{3} & 0 \\
v_{1 i} & v_{2 i} & v_{3 i} & m v_{i} \\
c_{1} & c_{2} & c_{3} & 0
\end{array}\right| \\
& =n \cdot\left|\begin{array}{lll}
u_{1} & u_{2} & u_{3} \\
v_{1} & v_{2} & v_{3} \\
c_{1} & c_{2} & c_{3}
\end{array}\right| f_{i k}-m\left|\begin{array}{cccc}
f_{1 i} & f_{2 i} & f_{3 i} & 0 \\
u_{1} & u_{2} & u_{3} & u_{k} \\
v_{1} & v_{2} & v_{3} & v_{k} \\
c_{1} & c_{2} & c_{3} & 0
\end{array}\right|-m\left|\begin{array}{llll}
f_{1 k} & f_{2 k} & f_{3 k} & 0 \\
u_{1} & u_{2} & u_{3} & u_{i} \\
v_{1} & v_{2} & v_{3} & v_{i} \\
c_{1} & c_{2} & c_{3} & 0
\end{array}\right| \text {. }
\end{aligned}
$$

Multiplicirt man nun die ersten drei Verticalreihen der beiden letzten Determinanten mit $\frac{x_{1}}{m-1}, \frac{x_{2}}{m-1}, \frac{x_{3}}{m-1}$, und zieht sie von der letzten Reihe ab, so verschwinden die Glieder der letzten Verticalreihe, bis auf das letzte, welches aber in Null multiplicirt wird. So verschwinden beide Determinanten, 
und es bleiben die $\Theta_{i k}$ den $f_{i k}$ proportional. Da nun das Product der Tangenten des Doppelpunktes in beiden Curven durch die Ausdrücke

$$
\Sigma \Sigma \Theta_{i k} X_{i} X_{k}, \quad f_{i k} X_{i} X_{k}
$$

dargestellt wird, so folgt hieraus unmittelbar der angegebene Satz.

Die Curve $\Theta=0$ ist von der Ordnung $3 n-7 ; \Theta=0$ und $f=0$ haben also $n(3 n-7)$ Schnittpunkte. Aber von diesen absorbirt dem Vorigen nach jeder der $\frac{n . n-3}{2}$ Doppelpunkte 6 , jeder der $n-2$ andern festen Punkte 2. Die Anzahl der übrig bleibenden Punkte beträgt also

$$
n(3 n-7)-3 n(n-3)-2(n-2)=4 .
$$

Die Function $Q$ ist also von der vierten Ordnung, und zugleich hat man den Satz:

Durch die $\frac{n . n-3}{2}$ Doppelpunkte der gegebenen Curve $n^{\text {ter }}$ Ordnung und durch $n-2$ feste Punkte derselben kann man vier Curven $(n-2)^{\text {ter }}$ Ordnung legen, welche die gegebene Curve berïhren.

Wesentlich ist noch, dass die Function $Q$ offenbar von den $a, b$ unabhängig ist. Der andere Factor der Discriminante enthält diese Grössen; und $P$ ist also für die $p$ vom ersten Grade, während es für $\lambda$ vom Grade $(n-2)$ ist. In der That stellt $P$, wenn man darin die $p$ als veränderliche Coordinaten ansieht, die Gleichung der Geraden dar, welche die beiden beweglichen Schnittpunkte verbindet. Sieht man aber die $p$ als gegeben, $\lambda$ als unbekannt an, so drückt dies den Satz aus:

Die Verbindungslinie der beweglichen Schnittpunkte, welche das System $u+\lambda v$ mit der gegebenen Curve gemein hat, umhïllt eine Curve $(n-2)^{\text {ter }}$ Classe.

\$. 4.

Weitere Behandlung der Ausdrücke der Coordinaten.

Aus dem Entwickelten geht hervor, dass man setzen kann

(14.) $W_{i k}=Q \cdot l_{i} l_{k}$,

wobei $Q$ eine Function vierten Grades, $l_{i}, l_{k}$ Functionen $(n-2)^{\text {ten }}$ Grades von $\lambda$ sind.

Nunmehr ist es auch möglich, die Ausdrücke (11.) der $x$ weiter zu transformiren. Setzt man zunächst für $\mu$ seinen Werth ein, und lässt den gemeinschaftlichen Nenner $B$ in $\rho$ eingehen, so findet man

Journal für Mathematik Bd. LXIV. Heft 8. 
218 Clebsch, ebene Curven, deren Coord. elliptische Funct. eines Parameters sind.

$$
\begin{aligned}
\boldsymbol{O} x_{i}= & B\left(\omega_{1 i} a_{1}+\omega_{2 i} a_{2}+\omega_{3 i} a_{3}\right)-C\left(\omega_{1 i} b_{1}+\omega_{2 i} b_{2}+\omega_{3 i} b_{3}\right) \\
& +P \sqrt{-Q}\left(\omega_{1 i} b_{1}+\omega_{2 i} b_{2}+\omega_{3 i} b_{3}\right) . \\
= & \frac{1}{2} \frac{\partial \cdot P^{2} Q}{\partial a_{i}}+P \sqrt{-Q}\left(\omega_{1 i} b_{1}+\omega_{2 i} b_{2}+\omega_{3 i} b_{3}\right) .
\end{aligned}
$$

Hier enthält nach Ausführung der Differentiation die rechte Seite offenbar $P \sqrt{-Q}$ als Factor, und indem man diesen abermals in $\varrho$ eingehen lässt, bleibt

$$
\text { (15.) } \quad \rho x=\omega_{1 i} b_{1}+\omega_{2 i} b_{2}+\omega_{3 i} b_{3}-\frac{\partial P}{\partial a_{i}} \sqrt{-Q} \text {. }
$$

Inzwischen ist aus der Vergleichung von (9.), (14.):

und daher endlich:

$$
P=l_{1} p_{1}+l_{2} p_{2}+l_{3} p_{3}=\left|\begin{array}{ccc}
l_{1} & l_{2} & l_{3} \\
a_{1} & a_{2} & a_{3} \\
b_{1} & b_{2} & b_{3}
\end{array}\right|
$$

$$
\text { (16.) }\left\{\begin{array}{l}
\varrho x_{1}=m_{11} b_{1}+\omega_{21} b_{2}+\omega_{31} b_{3}-\left(b_{2} l_{3}-b_{3} l_{2}\right) \sqrt{-Q} \\
\varrho x_{2}=\omega_{12} b_{1}+\omega_{22} b_{2}+\omega_{32} b_{3}-\left(b_{3} l_{1}-b_{1} l_{3}\right) \sqrt{-Q} \\
\varrho x_{3}=\omega_{13} b_{1}+\omega_{23} b_{2}+\omega_{33} b_{3}-\left(b_{1} l_{2}-b_{2} l_{1}\right) \sqrt{-Q}
\end{array}\right.
$$

Diese Gleichungen geben die Darstellung der $x$ als Functionen des Parameters $\lambda$. Sie ist rational, bis auf die Wurzel eines Ausdrucks $Q$ der vierten Ordnung; die rationalen Functionen in welche dieselbe multiplicirt ist, sind von der $(n-2)^{\text {ten }}$ Ordnung, und die übrigen Theile der $x$ sind rationale Functionen der $n^{\text {ten }}$ Ordnung. Die willkürlichen Grössen $a$ kommen darin nicht mehr vor; die $b$ nur noch in linearer Wejse.

Die Ausdrücke (16.) gestatten übrigens eine sehr einfache geometrische Deutung. Die Coefficienten von $/ Q$ śind nämlich die Coordinaten des Punktes, in welchem die willkürlich gewählte Gerade $b$ die Verbindungslinie $l$ der beweglichen Punkte triff; die ersten Theile der rechten Seiten sind die Coordinaten des Pols von $b$ in Bezug auf jenes Punktenpaar selbst, oder die Coordinaten des Punktes, welcher mit dem ersterwähnten und mit dem Paare selbst, zu welchem $\boldsymbol{x}$ gehört, ein harmonisches System bildet.

Aber die Ordnungen auf der rechten Seite von (16.) sind noch höher, als es die Natur der Sache erfordert. Man erkennt dies sofort, wenn man etwa den Durchschnitt einer beliebigen Geraden

$$
\text { (17.) } \quad c_{1} x_{1}+c_{2} x_{2}+c_{3} x_{3}=0
$$

mit der durch (16.) dargestellten Curve bestimmt. Diese Aufgabe muss auf 
eine Gleichung $n^{\text {ten }}$ Grades für $\lambda$ führen. Aber wenn man die Werthe der $x$ in (17.) einsetzt, so kommt:

$$
\left(17^{a} .\right) \quad \sum \Sigma \omega_{i k} b_{i} c_{k}=\sqrt{-Q} \cdot \Sigma \pm c_{1} b_{2} l_{3},
$$

und wenn man diese Gleichung rational macht, erhält man die Gleichung

$$
\text { (18.) } \quad\left(\Sigma \Sigma \omega_{i k} b_{i} c_{k}\right)^{2}+Q\left(\Sigma \pm c_{1} b_{2} l_{3}\right)^{2}=0 \text {, }
$$

welche für $\lambda$ von der Ordnung $2 n$ ist. Dieselbe enthält daher einen überflüssigen Factor; er ist leicht zu bestimmen. Nach den Formeln (9.), (14.) ist nämlich

$$
Q \cdot\left(\Sigma \pm c_{1} b_{2} l_{3}\right)^{2}=\sum \sum \omega_{i k} b_{i} b_{k i} . \Sigma \sum \omega_{i k} c_{i} c_{k}-\left(\sum \sum \omega_{i k} b_{i} c_{k}\right)^{2}
$$

und (18.) geht also über in

$$
0=\Sigma \Sigma \omega_{i k} b_{i} b_{k} . \Sigma \sum \omega_{i k} c_{i} c_{k} .
$$

Der wirklich dem Problem angehörige Factor ist also der Factor $n^{\text {ten }}$ Grades

$$
0=\Sigma \Sigma \omega_{i k} c_{i} c_{k},
$$

während der Factor

$$
0=\Sigma \Sigma \omega_{i k} b_{i} b_{k}
$$

als von der Geraden $c$ ganz unabhängig, nur Fremdartiges liefern kann. Dieser fremde Factor hat aber für die $b$ ganz die gleiche Beschaffenheit, wie der andere für die $c$, er liefert also die Schnittpunkte der Curve mit der willkürlich gegebenen Geraden $b$, und der zu hohe Grad der rechten Theile von (16.) erklärt sich dadurch, dass alle jene Ausdrücke noch für gewisse, mit den Schnittpunkten der Geraden $b$ mit der Curve zusammenhängende Werthe verschwinden.

§. 5 .

Einführung eines neuen Parameters, für welchen die Ausdrücke der Coordinaten ihre einfachste Form annehmen.

In welcher Weise man diese der Sache fremden Werthe entfernen kann, und wie die Gestalt der resultirenden Ausdrücke ist, übersieht man leicht mit Hülfe der Theorie der elliptischen Functionen. Ich denke mir für $\lambda$ einen linearen Ausdruck $\frac{\alpha+\beta \lambda}{\gamma+\delta \lambda}$ gesetzt, und die Constanten $\alpha, \beta, \gamma, \delta$ so bestimmt, dass der Zähler von $Q$, abgesehen von einem constanten Factor in $\lambda .1-\lambda .1-x^{2} \lambda$ übergeht. Man kann dies auch geometrisch dadurch ausdrücken, dass man an Stelle der bisher beliehig dem Curvenbüschel $(n-2)^{\text {ter }}$ Ordnung entnommenen Curven $u=0, v=0$ zwei solche treten lässt, welche die Curve $f=0$ 
berühren, so dass $\lambda=0$ und $\lambda=\infty$ Wurzeln von $Q=0$ werden. Dies vorausgesetzt sei nun

$$
V^{\prime} \lambda=\sin \operatorname{am} u, \quad \sqrt{1-\lambda}=\cos \mathrm{am} u, \quad \sqrt{1-\varkappa^{2} \lambda}=\Delta \mathrm{am} u .
$$

Jeder der Ausdrücke, welche die rechten Theile von (16.) bilden, hat also die Form

$$
F\left(\sin ^{2} \mathrm{am} u\right)+F_{1}\left(\sin ^{2} \mathrm{am} u\right) \cdot \frac{d \cdot \sin ^{2} \mathrm{am} u}{d u},
$$

und zwar ist dabei die Willkürlichkeit verschwunden, welche früher in dem zweifelhaften Vorzeichen der Wurzelgrösse lag; ein Paar auf derselben Curve des Systems $u+\lambda v=0$ gelegener Punkte wird in dieser Form durch die Argumente $u$ und $-u$ unterschieden.

Die angegebene Form lässt sich nach Hermite (Note zur dritten Ausgabe von Lacroix, p. 66) ersetzen durch einen Ausdruck von der Gestalt

$$
C \cdot \frac{H\left(u-\alpha^{\prime}\right) H\left(u-\alpha^{\prime \prime}\right) \ldots H\left(u-\alpha^{(2 n)}\right)}{\Theta^{2 n}(u)}
$$

wo

$$
\text { (19.) } \alpha^{\prime}+\alpha^{\prime \prime} \ldots \alpha^{(2 n)}=0 \text {, }
$$

und ebendies gilt nicht bloss für die rechten Theile von (16.) selbst, sondern für jede, einer beliebigen Geraden entsprechende Combination, sodass die für den Schnitt einer beliebigen Geraden $c_{1} x_{1}+c_{2} x_{2}+c_{3} x_{3}=0$ mit den Curven geltenden Bedingungen immer die Form annehmen

$$
H\left(u-\alpha^{\prime}\right) . H\left(u-\alpha^{\prime \prime}\right) \ldots H\left(u-\alpha^{(2 n)}\right)=0 .
$$

Hiebei ist ein Theil der $\alpha$, nämlich $n$ derselben, veränderlich, den veränderlichen Schnittpunkten der Geraden mit der Curve entsprechend; die $n$ übrigen $\alpha$ sind aber constant; sie führen auf diejenigen Werthe von $\lambda$, welche in den Schnittpunkten der Geraden $b$ mit der Curve $f=0$ eintreten, und sind also den dort stattindenden Werthen von $u$ gleich oder entgegengesetźt. Hierüber zu entscheiden gestattet die Gleichung $\left(17^{a}.\right)$. Wenn wir nämlich die Gerade $c$ mit der Geraden $b$ selbst zusammenfallen lassen, so gehen die $n$ ersten $\alpha$ unbedingt in diejenigen Werthe vou $u$ über, welche in den Schnittpunkten von $b$ mit $f=0$ stattinden. Weil aber für diesen Fall aús der entsprechenden Gleichung $\left(17^{a}.\right)$ für $\lambda$ die Wurzelgrösse herausfällt, und man nur eine Gleichung $n^{\text {ten }}$ Grades für $\lambda=\sin ^{2}$ am $u$ aufzulösen hat, so sind die ihr entsprechenden Werthe von $u$ paarweise gleich und entgegengesetzt, so dass, wenn die den Schnittpunkt von $b=0$ mit $f=0$ zugehörigen Werthe 
von $u$ mit $\beta^{\prime}, \beta^{\prime \prime}, \ldots \beta^{(n)}$ bezeichnet werden, die übrigen jener Gleichung genügenden Werthe $-\beta^{\prime},-\beta^{\prime \prime}, \ldots-\beta^{(n)}$ sind. Die Argumente $\alpha^{(n+1)}, \alpha^{(n+2)}, \ldots$ $\alpha^{(2 n)}$ müssen also mit diesen letzten Werthen übereingestimmt haben.

Lassen wir nur den bei jedem der $x$ auftretenden Factor

$$
\boldsymbol{H}\left(u+\boldsymbol{\beta}^{\prime}\right) \cdot \boldsymbol{H}\left(u+\boldsymbol{\beta}^{\prime \prime}\right) \ldots \boldsymbol{H}\left(u+\boldsymbol{\beta}^{(n)}\right)
$$

in $\varrho$ eingehen, so erhalten wir Formeln folgender Art:

$$
\text { (20.) }\left\{\begin{array}{l}
\varrho x_{1}=c_{1} \cdot \frac{H\left(u-\alpha_{1}^{\prime}\right) H\left(u-\alpha_{1}^{\prime}\right) \ldots H\left(u-\alpha_{1}^{(n)}\right)}{\Theta^{n}(u)}, \\
\varrho x_{2}=c_{2} \cdot \frac{H\left(u-\alpha_{2}^{\prime}\right) H\left(u-\alpha_{2}^{\prime \prime}\right) H \ldots\left(u-\alpha_{2}^{(n)}\right)}{\Theta^{n}(u)}, \\
\varrho x_{3}=c_{3} \cdot \frac{H\left(u-\alpha_{3}^{\prime}\right) H\left(u-\alpha_{3}^{\prime \prime}\right) H \ldots\left(u-\alpha_{3}^{(n)}\right)}{\Theta^{n}(u)} .
\end{array}\right.
$$

Die rechten Theile lassen sich hier nicht mehr unmittelhar mit Anwendung des Hermiteschen Satzes in algebraische Form zurückführen; denn die Summe der $\alpha$ ist nicht mehr Null. Vielmehr, setzen wir

$$
\beta^{\prime}+\beta^{\prime \prime}+\cdots+\beta^{(n)}=c,
$$

so ergiebt sich aus (19.), dass für die Schnittpunkte jeder Geraden mit der Curve auch

$$
\alpha^{\prime}+\alpha^{\prime \prime}+\cdots+\alpha^{(n)}=c .
$$

Es folgt also einerseits, dass die Summe der zu den Schnittpunkten einer Geraden mit der Curve gehörigen Argumente stets denselben constanten Werth besitzt, ein Satz, den wir weiter unten in viel allgemeinerer Form wieder auftreten sehen werden. Andrerseits aber liegt es nahe, wenn $c$ diesen constanten Werth bezeichnet, an Stelle von $u$ die Variable

$$
u^{\prime}=u-\frac{c}{n},
$$

und an Stelle der $\alpha$ die constanten Werthe

$$
\beta=\alpha-\frac{c}{n}
$$

einzuführen, wodurch die Formeln (20.) ungeändert bleiben. Nunmehr ist die Summe der $\beta$ immer gleich Null, und nach demselben Hermiteschen Satz, von welchem bereits oben Gebrauch gemacht wurde, kann man den rechten Theilen von (20.) jetzt die Form geben

$$
C .\left\{f\left(\lambda^{\prime}\right)+\sqrt{\lambda^{\prime} .1-\lambda^{\prime} .1-k^{2} \lambda^{\prime}} \cdot \varphi\left(\lambda^{\prime}\right)\right\}
$$

wenn $n$ gerade ist, oder die Form

$$
C .\left\{f\left(\lambda^{\prime}\right) \sqrt{\lambda^{\prime}+} \sqrt{1-\lambda^{\prime} .1-k^{2} \lambda^{\prime}} \cdot \varphi\left(\lambda^{\prime}\right)\right\}
$$


222 Clebsck, ebene Curven, deren Coord. elliptische Funct. eines Parameters sind.

wenn $n$ eine ungerade Zahl ist. Und zwar ist dabei im ersten Falle $f$ von der Ordnung $\frac{n}{2}, \varphi$ von der Ordnung $\frac{n}{2}-2$, im zweiten Falle ist $f$ von der Ordnung $\frac{n-1}{2}, \varphi$ von der Ordnung $\frac{n-3}{2}$. Die Variable $\lambda^{\prime}$ aber hat die Bedeutung

$$
\sqrt{ } \lambda^{\prime}=\sin a \dot{m}\left(u-\frac{c}{n}\right)=\frac{\sqrt{\lambda .1-\epsilon .1-k^{2} \varepsilon}-\sqrt{\varepsilon .1-\lambda .1-k^{2} \lambda}}{1-k^{2} \varepsilon \lambda},
$$

wenn

$$
\text { (22.) } \gamma^{\prime} \varepsilon=\sin \operatorname{am} \frac{c}{n}
$$

gesetzt ist. Man ist also von den Formeln (16.) zu den reducirten Formeln übergegangen mittels einer irrationalen Substitution (21.), welche die Entfernung der in (16.) enthaltenen überflüssigen Factoren ermöglicht.

Das Endresultat dieser Untersuchung lässt sich etwas bequemer aussprechen, wenn man für $\lambda^{\prime}$ wieder einen linearen Ausdruck $\frac{\alpha+\beta z}{\gamma+\delta z}$ einführt. Hiedurch treten nach Entfernung der Nenner an Stelle der Factoren der Wurzelgrösse wieder allgemein lineare Ausdrücke $p+q z$, und man kann folgenden Satz aussprechen:

Die Coordinaten eines Punkts einer Curve $n^{\text {ter }}$ Ordnung mit $\frac{n . n-3}{2}$ Doppelpunkten lassen sich immer in der Form darstellen:

$$
\text { (23.) }\left\{\begin{array}{l}
\varrho x_{1}=f_{1}(z) \sqrt{ } M+\varphi_{1}(z) \sqrt{ }, \\
\rho x_{2}=f_{2}(z) \sqrt{ } \boldsymbol{M}+\varphi_{2}(z) \sqrt{ }, \\
\varrho x_{3}=f_{3}(z) \sqrt{ } \boldsymbol{M}+\varphi_{3}(z) \sqrt{ },
\end{array}\right.
$$

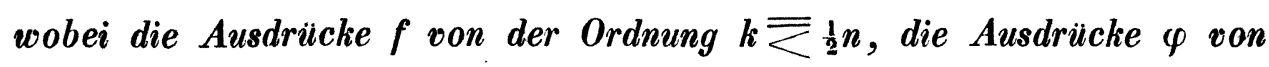
der Ordnung $h \bar{\sum} \frac{1}{2} n$, wobei ferner $M$ von der Ordnung $n-2 k, N$ von der Ordnung $n-2 h$ und $k+h=n-2$ ist, so dass $M$. $N$ immer ein Ausdruck vierter Ordnung wird; und zwar kann man immer einer der beiden Functionen $M, N$ die Ordnung 0 , der anderen die Ordnung 4, oder einer die Ordnung 1, der anderen die Ordwung 3 geben.

\section{6.}

Andere Hülfssysteme. Der Modul ist von der Wahl der festen Punkte unabhängig.

Der Curvenbüschel $(n-2)^{\text {ter }}$ Ordnung $u+\lambda \bullet=0$, welcher hier benutzt wurde, zeichnet sich dadurch aus, dass er der Büschel niedrigster Ordnung 
ist, welcher zu dem vorliegenden $Z$ wecke brauchbar ist, und zugleich durch alle Doppelpunkte geht. Der Curvenbüschel niedrigster Ordnung, an den man überhaupt Betrachtungen wie die vorliegenden anknüpfen kann, ist von der $n-3^{\text {ien }}$ Ordnung; man muss aber bei ihm als Grundpunkte die Doppelpunkte der gegehenen Curven mit Ausschluss eines derselben ansehen. Auch hat dieser Büschel den Nachtheil, dass er bei den Curven dritter Ordnung, für welche die Anzahl der Doppelpunkte Null wird, nicht mehr besteht, während der oben gebrauchte die Substitution des Herrn Aronhold (Monatsbericht der Berliner Academie, 25. April 1861) liefert. Man kann aber genau mit demselben Erfolge und mit der gleichen Allgemeinheit einen Curvenbüschel $n-1^{\text {ter }}$ Ordnung anwenden, welcher durch die Doppelpunkte und durch $2(n-1)$ beliebig auf der Curve $f=0$ gewählte feste Punkte hindurchgeht.

Wir haben schon oben gesehen, dass der Modul $k$ der hier auftretenden elliptischen Functionen allein von der Curve $f=0$ und von den Constanten des Büschels abhängen kann, d. h. von den Coordinaten der beliebigen n-2 Punkte, welche man zu Grundpunkten des Büschels gemacht hat. Es ist leicht ${ }^{-} z$ zeigen, dass er auch von diesen letzten nicht abhängig ist. Bilden wir nämlich das Integral

$$
\int \frac{\theta \cdot \Sigma \pm c_{1} x_{2} d x_{3}}{c_{1} f_{1}+c_{2} f_{2}+c_{3} f_{3}}
$$

wobei die Variabeln $x_{1}, x_{2}, x_{3}$ durch die Gleichung $f=0$ verbunden sein sollen, und wo $\theta=0$ die Gleichung der Curve $(n-3)^{\text {ter }}$ Ordnung ist, welche durch die Doppelpunkte hindurchgeht. Dieses Integral kann man mit Benutzung der Werthe (23.) in ein elliptisches Integral nach $\lambda$ verwandeln, und da die zu integrirende Function nirgends von einer höheren als von der $\frac{1}{2}^{\text {terr }}$ Ordnung unendlich wird, so ist es nothwendig ein Integral erster Gattung, d. h. es geht über in

$$
C \cdot \int \frac{d \lambda}{\sqrt{\lambda .1-\lambda .1-k^{2} \lambda}} .
$$

Wenn wir nun die $n-2$ beliebigen Grundpunkte des Büschels verändern, so mag ein nener Modul $k_{1}$ und eine neue Constante $C_{1}$ entstehen. Es ist also einerseits

$$
C \cdot \int \frac{d \lambda}{\sqrt{\lambda .1-\lambda .1-k^{2} \lambda}}=C_{1} \cdot \int \frac{d \lambda_{1}}{\sqrt{\lambda_{1} \cdot 1-\lambda_{1} \cdot 1-k^{2} \lambda_{1}}},
$$

andererseits folgt aus der doppelten Darstellungsweise der $\alpha$, und daraus, dass $\lambda$ und $\lambda_{1}$ als rationale Functionen der $x$ darstellbar sind, dass $\lambda$ sich ebenso- 
224 Clebsch, ebene Curven, deren Coord. elliptische Funct. eines Parameters sind.

wohl durch $\lambda_{1}$ und $\sqrt{\lambda_{1} .1-\lambda_{1} .1-k_{1}^{2} \lambda_{1}}$, als $\lambda_{1}$ durch $\lambda$ und $\sqrt{\lambda .1-\lambda .1-k^{2} \lambda}$ rational ausdrücken lässt. Dies ist nur möglich, wenn $k_{1}$ einer der zu $k$ conjugirten Module $k^{\prime}, \frac{1}{k}$ etc. ist; und da eben die Einführung der letztern nichts als eine veränderte Benutzung der vier Wurzeln von $Q=0$ anzeigt, so kann man es immer so einrichten, dass $k_{1}=k$ wird, und man sieht also, dass $k$ nothwendig von der Lage der $n-2$ gewählten Grundpunkte unabhängig ist.

Dieses Resultat, nach welchem $k$ eine für die Curve $f=0$ charakteristische Constante ist, erlaubt eine geometrische Deutung, welche eine von Herrn Salmon (Bd. 42, p. 274 dieses Journals) zuerst gegebene Eigenschaft der Curven dritter Ordnung in merkwürdiger Weise verallgemeinert. Denken wir uns nämlich die vier Curven des Büschels $u+\lambda v$, welche $f=0$ berühren, und in irgend einem gemeinsamen Punkte derselben die Tangenten gelegt, so ist das Doppelverhältniss der vier Tangenten, was auch kurz das Doppelverhältniss der vier Curven heissen mag, gleich dem Doppelverhältniss der vier entsprechenden $\lambda$, oder der Wurzeln von $Q$, also nur von $k$ abhängig, mithin unabhängig von der Lage der $n-2$ gewählten Grundpunkte. Und so hat man den Satz:

Wählen wir auf einer Curve ${ }^{\text {ter }}$ Ordnung mit $\frac{n . n-3}{2}$ Doppelpunkten $n-2$ beliebige Punkte, und legen durch sie und die Doppelpunkte die vier Curven $n-2^{\text {ter }}$ Ordnung, welche die gegebene Curve ausserdem noch berïhren. Dann ist das Doppelverhältniss dieser vier Curven immer das gleiche, wo man auch jene $n-2$ Punkte angenommen habe.

Bei Curven dritter Ordnung ist dies der oben angedeutete Satz, dass das von einem Punkt der Curve gelegte Tangentenbüschel stets dasselbe Doppelverhältniss habe.

Obiger Satz gilt auch noch, wenn man statt der Curven $n-2^{\text {ter }}$ Ordnung immer Curven $(n-1)^{\text {ter }}$ Ordnung und statt der $n-2$ gewählten Grundpunkte deren immer $2(n-1)$ substituirt.

\section{\$. 7.}

Untersuchung der Curven, welche durch die Formeln (23.) dargestellt werden. Sie sind immer von der $n^{\text {ten }}$ Ordnung und haben $\frac{n \cdot n-3}{2}$ Doppelpunkte.

Ich stelle mir jetzt umgekehrt die Aufgabe, alle Curven zu untersuchen, die durch Gleichungen von der Form (23,) dargestellt sind. Dabei 
darf man voraussetzen, dass weder $M$ einen sämmtlichen $\varphi$ gemeinsamen Factor enthält, noch $N$ einen sämmtlichen $f$ gemeinsamen; und dass überhaupt die Grössen $f_{i}^{2} M-\varphi_{i}^{2} N$ keinen allen gemeinschaftlichen Factor enthalten. Man erkennt dann leicht, dass die durch (23.) dargestellte Curve von der $n^{\text {ten }}$ Ordnung ist und $\frac{n \cdot n-3}{2}$ Doppelpunkte besitzt. Hiedurch wird die völlige Identität beider Definitionen festgestellt, so dass es gleichgültig ist, ob man die Curve durch die Ordnung und die Zahl ihrer Doppelpunkte, oder ob man sie durch die Form (23.) characterisirt.

Was zunächst die Ordnung der Curve (23.) betrifft, so wird dieselbe ermittelt, indem man in den Ausdruck einer Geraden $c_{1} x_{1}+c_{2} x_{2}+c_{3} x_{3}=0$ jene Werthe der $x$ substituirt, und die Anzahl der Schnittpunkte der Geraden mit der Curve ermittelt. Man findet die Gleichung

$$
\left(c_{1} f_{1}+c_{2} f_{2}+c_{3} f_{3}\right)^{2} M-\left(c_{1} \varphi_{1}+c_{2} \varphi_{2}+c_{3} \varphi_{3}\right)^{2} N=0 .
$$

Dieselbe ist für $z$ von der $n^{\text {ten }}$ Ordnung, daher auch $n$ die Ordnung der Curve, da das Auftreten von den $c$ unabhängiger Factoren ausgeschlossen wurde.

Die Doppelpunkte der Curve (23.) bestimmen sich dadurch, dass für zwei verschiedene Werthe $z, z^{\prime}$, die dem Doppelpunkt im Verlauf der sich in ihm schneidenden Zweige entsprechen, proportionale Werthe der Coordinaten herauskommen müssen.

Man hat also ein zusammengehöriges Werthepaar $z, z^{\prime}$ aus den Gleichungen zu bestimmen:

$$
\left\{\begin{array}{l}
f_{1} \sqrt{ } \boldsymbol{M}+\varphi_{1} \sqrt{ } \boldsymbol{N}=\sigma\left(f_{1}^{\prime} \sqrt{ } \boldsymbol{M}^{\prime}+\varphi_{1}^{\prime} \sqrt{ } \boldsymbol{N}^{\prime}\right) \\
f_{2} \sqrt{ } \boldsymbol{M}+\varphi_{2} \sqrt{ } \boldsymbol{N}=\sigma\left(f_{2}^{\prime} \sqrt{ } \boldsymbol{M}^{\prime}+\varphi_{2}^{\prime} \sqrt{ } \boldsymbol{N}^{\prime}\right) \\
f_{3} \sqrt{ } \boldsymbol{M}+\varphi_{3} \sqrt{ } \boldsymbol{N}=\sigma\left(f_{3}^{\prime} \sqrt{ } \boldsymbol{M}^{\prime}+\varphi_{3}^{\prime} \sqrt{ } \boldsymbol{N}^{\prime}\right)
\end{array}\right.
$$

wo die obern Striche auf das Argument $z^{\prime}$ hinweisen sollen. Werden nun die Unterdeterminanten, welche man durch Weglassung einer Verticalreihe aus dem unvollständigen System

$$
\begin{array}{llll}
f_{1} & \varphi_{1} & f_{1}^{\prime} & \varphi_{1}^{\prime}, \\
f_{2} & \varphi_{2} & f_{2}^{\prime} & \varphi_{2}^{\prime}, \\
f_{3} & \varphi_{3} & f_{3}^{\prime} & \varphi_{3}^{\prime},
\end{array}
$$

erhält, dividirt durch $z-z^{\prime}$, durch

$$
\boldsymbol{F}, \boldsymbol{\Phi}, \boldsymbol{F}^{\prime}, \boldsymbol{\Phi}^{\prime}
$$

bezeichnet, so folgt aus (24.) das folgende aequivalente System : 


$$
\begin{aligned}
& \sqrt{ } M: \sqrt{ } N: \sigma \sqrt{ } \boldsymbol{M}^{\prime}: \sigma \sqrt{ } \boldsymbol{N}^{\prime} \\
= & \boldsymbol{F}: \boldsymbol{\Phi}: \quad \boldsymbol{F}^{\prime}: \boldsymbol{\Phi}^{\prime} .
\end{aligned}
$$

oder

$$
\left\{\begin{array}{l}
F^{2} N-\Phi^{2} M=0=\Omega\left(z, z^{\prime}\right) \\
F^{\prime 2} N^{\prime}-\Phi^{\prime 2} M^{\prime}=0=\Omega\left(z^{\prime}, z\right)
\end{array} .\right.
$$

Beide Gleichungen unterscheiden sich nur durch Vertauschung von $z$ mit $z$; beide sind für das erste Argument vom Grade $n-2$, für das zweite vom Grade $2 n-6$. Die Gleichung, welche nach Elimination von $z^{\prime}$ übrig bleibt, ist nach Abel durch $\Omega(z, z)$ theilbar, und der Rest also vom Grade

$$
(n-2)^{2}+(2 n-6)^{2}-3 n-8=(5 n-16)(n-3) \text {. }
$$

Aber auch noch die so erhaltene Gleichung besitzt einen überflüssigen Factor. Denn die Gleichungen (25.) bedingen rückwärts die Gleichungen (24.) nur dann, wenn nicht $\boldsymbol{F}, \Phi, \boldsymbol{F}^{\prime}, \Phi^{\prime}$ gleichzeitig verschwinden. Dies kann in der That geschehen; und ein davon herrührender Factor muss noch in der letzten Gleichung unterdrückt werden. Betrachten wir die Gleichungen (25.) als Gleichungen zweier Curven mit den Coordinaten $z$, $z^{\prime}$, so sind für dieselben diejenigen Punkte, in denen $\boldsymbol{F}, \Phi, F^{\prime}, \Phi^{\prime}$ gleichzeitig verschwinden, Doppelpunkte; ist die Zahl solcher Punkte gleich $\rho$, so erniedrigt sich die Anzahl der übrigen Schnittpunkte um 4@, und der Grad der oben erwähnten Endgleichung reducirt sich auf

$$
(5 n-16)(n-3)-4 \rho .
$$

Es kommt also nur noch darauf an, die Anzahl von Lösungen zu bestimmen, welche die Gleichungen

$$
F=0, \quad \Phi=0, \quad F^{\prime}=0, \quad \Phi^{\prime}=0
$$

gemeinsam haben. Aber die dritte und vierte Gleichung ist immer eine Folge der beiden ersten. Denn da der Entstehung dieser Ausdrücke nach identisch

$$
\boldsymbol{F} f_{i}+\boldsymbol{\Phi} \varphi_{i}+\boldsymbol{F}^{\prime} f_{i}^{\prime}+\boldsymbol{\Phi}^{\prime} \varphi_{i}^{\prime}=\mathbf{0}
$$

so finden für $\boldsymbol{F}=0, \boldsymbol{\Phi}=0$ immer die Gleichungen statt:

$$
\begin{aligned}
& \boldsymbol{F}^{\prime} f_{1}^{\prime}+\boldsymbol{\Phi}^{\prime} \varphi_{1}^{\prime}=\mathbf{0}, \\
& \boldsymbol{F}^{\prime} f_{2}^{\prime}+\boldsymbol{\Phi}^{\prime} \varphi_{2}^{\prime}=\mathbf{0}, \\
& \boldsymbol{F}^{\prime} \boldsymbol{f}_{3}^{\prime}+\boldsymbol{\Phi}^{\prime} \varphi_{3}^{\prime}=\mathbf{0},
\end{aligned}
$$

aus denen im Allgemeinen immer $F^{\prime}=0, \Phi^{\prime}=0$ folgt. Es ist also $\rho$ die Anzahl von Lösungen, welche den Gleichungen $F=0, \Phi=0$ gemeinsam sind. 
Nun sind die Ordnungen dieser Ausdrücke

$$
\begin{aligned}
& \text { für } z: h-1 \text { und } k-1, \\
& \text { für } z^{\prime}: h+k-1 ;
\end{aligned}
$$

daher kommt nach Elimination von z eine Gleichung vom Grade $(h+k-1)(h+k-2)$ $=(n-3)(n-4)$. Dies also ist der Werth von $\varrho$, und der Grad der zu bestimmenden Endgleichung ist

$$
(5 n-16)(n-3)-4(n-3)(n-4)=n . n-3 .
$$

Dies stimmt mit der Existeuz von $\frac{n . n-3}{2}$ Doppelpunkten überein. Denn da jedem Doppelpunkte zwei Argumente zukommen, so müssen n.n-3 Argumente gefunden werden. Die Gleichung $n . n-3^{\text {ten }}$ Grades aber hat die Eigenschaft, dass aus (25.) immer eine Wurzel $z^{\prime}$ eine rationale Function von $z$, und $z$ dieselbe rationale Function von $z^{\prime}$ wird. Man löst also jene Gleichung mit Hülfe einer Gleichung vom Grade $\frac{n . n-3}{2}$, und von $\frac{n . n-3}{2}$ quadratischen Gleichungen.

So ist nachgewiesen, dass die Curve $n^{\text {ter }}$ Ordnung, deren Coordinaten die Form (23.) annehmen können, immer $\frac{n . n-3}{2}$ Doppelpunkte besitzt, und also immer von der Eingangs betrachteten Art ist. Von diesen Doppelpunkten können einige in Rückkehrpunkte übergehen: dies tritt ein, sobald die Grössen $z, z^{\prime}$ eines zu einem Doppelpunkte gehörigen Paares einander gleich werden.

\section{\$. 8 .}

Bedingungen für das System der Schnittpunkte der gegebenen mit einer andern algebraischen Curve.

Wenn eine Curve $m^{\text {ter }}$ Ordnung die gegebene Curve schneidet, so treten zwischen den Parametern der $m n$ Schnittpunkte gewisse nothwendige Beziehungen ein, welche von der Natur der Curve $m^{\text {ter }}$ Ordnung unabhängig sind, und nur von der Zahl $m$ selbst abhängen. Das Abelsche Theorem lehrt diese Bedingungen kennen. Man weiss, dass die Anzahl derselben $\frac{n-1 . n-2}{2}$ ist, sobald $m$ grösser als $n-3$ wird, während sie für kleinere Werthe von m sich vermindert. Diese Bedingungen sollen nun aufgestellt werden.

Denken wir uns die Gleichung irgend einer Curve $m^{\text {ter }}$ Ordnung gegeben, deren Durchschnitt mit der vorliegenden Curve untersucht werden soll. Führen wir in diese Gleichung die Ausdrückè (23.) ein, so haben wir eine 30 * 
Gleichung in $z$, welche die gesuchten Schnittpunkte liefert. Diese Gleichung hat, jenachdem $m$ ungrade oder gerade ist, eine der Formen

$$
P \sqrt{M}+Q_{\sqrt{ }} N=\mathbf{0}, \quad P+Q_{\sqrt{M N}}=\mathbf{0},
$$

wo $P, Q$ rationale Functionen von $\lambda$ sind. Ich will diese beiden Formen zusammen durch

$$
\text { (26.) } \quad P_{V} U+Q_{V} V=0
$$

bezeichnen, wo denn immer $U V=M N$. Die rationale Gleichung in $z$ ist dann $P^{2} U-Q^{2} V=0$, und nennt man die Parameter der Schnittpunkte $z_{1}, z_{2}, \ldots z_{m n}$, so ist immer

$$
\left(26^{a} .\right) \quad P^{2} U-Q^{2} V=K . z-z_{1} \cdot z-z_{2} \ldots z-z_{m n} .
$$

Denken wir uns die Curve $m^{\text {ter }}$ Ordnung beweglich, so dass auch das Schnittpunktsystem sich continuirlich ändert, so folgt aus dieser Gleichụng

$$
\text { (27.) } \sum_{i=1}^{i=m n} \int \frac{d z_{i}}{z-z_{i}}=-\log \frac{P^{2} U-Q^{2} V}{K}+\text { Const. }
$$

Aber das Abelsche Theorem (Bd. III, p. 314 dieses Journals) liefert aus (26 ${ }^{a}$.) auch noch folgende Gleichung:

$$
\sum_{i=1}^{i=m n} \int \frac{\sqrt{M N} \cdot d z_{i}}{\left(z-z_{i}\right) \sqrt{\bar{M}_{i} \bar{N}_{i}}}=\log \frac{P \sqrt{U}-Q \sqrt{V}}{P \sqrt{U}+Q \sqrt{\bar{V}}}+\text { Const., }
$$

so dass aus (27.), (28.) die Combination folgt:

$$
\sum_{i=1}^{i=m n} \int \frac{\sqrt{M_{i} \bar{N}_{i}}+\sqrt{M N}}{\left(z-z_{i}\right) \sqrt{M_{i} \bar{N}_{i}}} d z_{i}=-2 \log \frac{P \sqrt{U}+Q \sqrt{V}}{K}+\text { Const. }
$$

Diese Gleichungen sind für $z$ identisch, so dass man dieser bei den Integrationen als constant betrachteten Grösse noch jeden beliebigen Werth beilegen kann. Setzen wir zunächst in (28.) $z=\infty$; dann ergiebt sich das Additionstheorem für die Integrale erster Gattung:

$$
\text { (30.) } \quad \sum_{i=1}^{i=m n} \frac{d z_{i}}{\sqrt{M_{i} N_{i}}}=\text { Const. }
$$

Bezeichnen wir ferner durch $a, b$ das Parameterpaar irgend eines Doppelpunktes. Nach (24.) finden dann immer Gleichungen von folgender Form statt:

$$
\left(f_{i} \sqrt{ } M+\varphi_{i} / N\right)_{z=a}=c \cdot\left(f_{i} / M+\varphi_{i} / N\right)_{z=b} .
$$

Der Ausdruck $P \sqrt{ } U+Q \sqrt{V}$ also, welcher nichts Anderes ist, als die Gleichung der Curve $m^{\text {ter }}$ Ordnung, wenn darin die $x_{i}$ durch die Ausdrücke $f_{i} \sqrt{M}+\varphi_{i} \sqrt{ } N$ ersetzt werden, erfüllt die Gleichung

$$
\left(P \sqrt{ }+Q_{V} V\right)_{z=a}=c^{m} \cdot(P \sqrt{ } U+Q V V)_{z=b} .
$$


Diese Gleichung kann man benutzen um aus (29.) eine weitere Reihe von Combinationen $\mathrm{zu}$ bilden, welche von den Coefficienten der Curve $m^{\text {ter }}$ Ordnung vollkommen unabhängig sind. Denn bildet man die Gleichung (29.) einmal für $z=a$, das andere Mal für $z=b$, so giebt die Differenz beider Gleichungen

$$
\sum_{i=1}^{i=m n} \int\left\{\frac{\sqrt{M_{i}} \bar{N}_{i}+\sqrt{M_{a} N_{a}}}{\left(a-z_{i}\right) \sqrt{M_{i} \bar{N}_{i}}}-\frac{\sqrt{M_{i} N_{i}}+\sqrt{M_{b} \bar{N}_{b}}}{\left(b-z_{i}\right) \sqrt{M_{i} N_{i}}}\right\} d z_{i}=\text { Const. }
$$

oder, wenn man will, gleich Const. $-2 m \log c$, was dasselbe sagt, da $c$ von den Coefficienten der Curve $m^{\text {ter }}$ Ordnung unabhängig ist. Solcher Gleichungen wie (31.) gieht es $\frac{n . n-3}{2}$, den verschiedenen Doppelpunkten entsprechend; diese, mit (30.) zusammen, bilden das vollständige System von $\frac{n-1 . n-2}{2}$ Bedingungen, denen das Schnittpunktsystem zu genügen hat. Es ergiebt sich also der Satz:

Wenn $a_{1}, b_{1} ; a_{2}, b_{2} ; \ldots a_{\frac{n . n-3}{2}}, b_{\frac{n . n-3}{2}}$ die Parameterpaare der Doppelpunkte sind, so müssen die Parameter $z_{1}, z_{2}, \ldots z_{m n}$ solcher Punkte der Curve (23.), welche zugleich auf einer Curve $m^{\text {ter }}$ Ordnung liegen, den $\frac{n-1 . n-2}{2}$ Bedingungen genügen:

$$
\left\{\begin{array}{c}
\sum_{i=1}^{i=m n} \int \frac{d z_{i}}{\sqrt{M_{i} N_{i}}}=\text { Const. } \\
\sum_{i=1}^{\sum} \int\left\{\frac{\sqrt{M_{i} N_{i}}+\sqrt{M_{a} N_{a}}}{\left(a-z_{i}\right) \sqrt{M_{i} N_{i}}}-\frac{\sqrt{M_{i} N_{i}}+\sqrt{M_{b} N_{b}}}{\left(b-z_{i}\right) \sqrt{M_{i} N_{i}}}\right\} d z_{i}=\text { Const. } \\
\left(a, b=a_{1}, b_{1} ; a_{2}, b_{2} ; \ldots a_{\frac{n, n-3}{2}}, \frac{b_{n, n-3}}{2}\right)
\end{array}\right.
$$

wobei die Werthe der Constanten nur noch von der Natur der gegebenen Curve selbst und von der Zahl $m$ abhängen können.

S. 9.

Einführung der elliptischen Functionen in die Bedingungen für das Schnittpunktsystem.

Die Bedingungsgleichungen (I.) können einfacher dargestellt werden, indem man an Stelle der Parameter $z$ die zugehörigen elliptischen Integrale erster Gattung als Argumente einführt. Denken wir uns wieder statt z einen linearen Ausdruck in $z$ gesetzt, und seine Coefficienten so bestimmt, dass 
230 Clebsch, ebene Curven, deren Coord. elliptische Funct. eines Parameters sind.

$\sqrt{M N}$ bis auf einen constanten Factor in $\sqrt{z .1-z .1-k^{2} z}$ übergeht, und setzen wir dann

$$
z=\sin ^{2} \mathrm{am} u, \quad a=\sin ^{2} \mathrm{am} \alpha, \quad b=\sin ^{2} \mathrm{am} \beta,
$$

so verwandeln sich die Gleichungen (I.) in

$$
\begin{aligned}
& u_{1}+u_{2}+\cdots+u_{m n}=\text { Const., } \\
& \sum_{i=1}^{i=m n^{\prime}}\left\{\log \frac{\sin ^{2} \mathrm{am} \beta-\sin ^{2} \mathrm{am} u_{i}}{\sin ^{2} \mathrm{am} \alpha-\sin ^{2} \mathrm{am} u_{i}}+2 \int\left[\frac{\operatorname{sinam} \alpha \operatorname{cosam} \alpha \Delta \mathrm{am} \alpha}{\sin ^{2} \mathrm{am} \alpha-\sin ^{2} \mathrm{am} u_{i}}-\frac{\operatorname{sinam} \beta \operatorname{cosam} \beta \Delta \mathrm{am} \beta}{\sin ^{2} \mathrm{am} \beta-\sin ^{2} a m u_{i}}\right] d u_{i}\right\} \\
& =\text { Const. }
\end{aligned}
$$

Die letzte Formel aber verwandelt sich nach Jacobi (dieses Journal Bd. 39, p. 348) in

Const. $=\Sigma\left\{\log \frac{\sin ^{2} a m \beta-\sin ^{2} a m u_{i}}{\sin ^{2} a m \alpha-\sin ^{2} a m u_{i}}-2 u_{i}\left(\frac{\Theta^{\prime} \alpha}{\Theta \alpha}-\frac{\Theta^{\prime} \beta}{\Theta \beta}\right)+\log \frac{H\left(\alpha+u_{i}\right) H\left(\beta-u_{i}\right)}{H\left(\alpha-u_{i}\right) H\left(\beta+u_{i}\right)}\right\} ;$ und wenn man das Glied

$$
-2\left(\frac{\Theta^{\prime} \alpha}{\Theta \alpha}-\frac{\Theta^{\prime} \beta}{\Theta \beta}\right) \Sigma u_{i}
$$

als wegen der ersten Gleichung constant in die Constante eingehen lässt, wenn man sodann die Formel

$$
\frac{\sin ^{2} a m \beta-\sin ^{2} a m u_{i}}{\sin ^{2} a \operatorname{mo}-\sin ^{2} a \operatorname{m} u_{i}}=\frac{\Theta^{2} \alpha}{\Theta^{2} \beta} \cdot \frac{H^{2} \beta \Theta^{2} u_{i}-\Theta^{2} \beta H^{2} u_{i}}{H^{2} \alpha \Theta^{2} u_{i}-\Theta^{2} \alpha H^{2} u_{i}}=\frac{\Theta^{2} \alpha}{\Theta^{2} \beta} \cdot \frac{H\left(\beta+u_{i}\right) H\left(\beta-u_{i}\right)}{H\left(\alpha+u_{i}\right) H\left(\alpha-u_{i}\right)}
$$

anwendet, erhält man endlich:

oder auch

$$
\sum_{i=1}^{i=m n} \log \frac{H\left(\alpha-u_{i}\right)}{H\left(\beta-u_{i}\right)}=\text { Const., }
$$

$$
\frac{H\left(\alpha-u_{1}\right) H\left(\alpha-u_{2}\right) \ldots H\left(\alpha-u_{m n}\right)}{H\left(\beta-u_{1}\right) H\left(\beta-u_{2}\right) \ldots H\left(\beta-u_{m n}\right)}=\text { Const. }
$$

Und so finden sich die Formeln (I.) ersetzt durch das System:

$$
\text { (II.) }\left\{\begin{array}{c}
u_{1}+u_{2}+\cdots+u_{m n}=c, \\
\frac{H\left(\alpha_{h}-u_{1}\right) \cdot H\left(\alpha_{h}-u_{2}\right) \ldots H\left(\alpha_{h}-u_{m n}\right)}{H\left(\beta_{h}-u_{1}\right) \cdot H\left(\beta_{h}-u_{2}\right) \cdots H\left(\beta_{h}-u_{m n}\right)}=c_{h}, \\
\left(h=1,2, \cdots \frac{n . n-3}{2}\right) .
\end{array}\right.
$$

Die erste dieser Gleichungen hätte man auch unmittelbar aus dem Theorem des Hrn. Hermite schliessen können, und zugleich hätte man die Constante c gleich Null gefunden. Nach jenem Satze wird nämlich immer, wenn $z=\sin ^{2}$ am $u$ gesetzt wird, für $u$ identisch

$$
P \sqrt{U}+Q_{V} V=C . e^{-\frac{v_{i j \pi u}}{K}} \cdot \frac{H\left(u-u_{1}\right) H\left(u-u_{2}\right) \ldots H\left(u-u_{m n}\right)}{\Theta^{m n}(u)},
$$


und zugleich ist

$$
u_{1}+u_{2}+\cdots+u_{m n} \equiv 0
$$

wenn durch das Congruenzzeichen angedeutet ist, dass linke und rechte Seite sich nur um ganze Vielfache der Perioden $2 \mu K+2 \nu i K^{\prime}$ unterscheiden sollen. Dies giebt offenbar die erste Formel der Systeme I., II., und zwar findet sich dabei $c \equiv \mathbf{0}$.

Um die anderen $c$ zu bestimmen, bemerke ich zunächst, dass, wenn die einzelnen $u_{k}$ um $2 \mu_{k} K+2 \nu_{k} i K^{\prime}$ wachsen, und dadurch ihre Summe um $2 \mu K+2 v i K^{\prime}$ vermehrt wird, die linke Seite der zweiten Gleichung (II.) um den Factor $e^{-\frac{\pi i v}{K}\left(\alpha_{h}-\beta_{h}\right)}$ zunimmt (vgl. das unten über die Perioden Gesagte). Daher ist es zweckmässig auch hier das Congruenzzeichen anzuwenden; dann kann man die $c_{h}$ als absolut bestimmt ansehen, und die Logarithmen beider Seiten der zweiten Gleichung (II.) können sich noch um $2 m_{h} i \pi-\frac{\pi i v}{K}\left(\alpha_{h}-\beta_{h}\right)$ unterscheiden. Die Gleichungen (II.) gelten, wie auch die Curve $m^{\text {ter }}$ Ordnung in éinfachere Curven zerfallen möge. Denken wir uns nun die Curve $m^{\text {ter }}$ Ordnung, um deren Schnittpunktsystem es sich handelt, in $m$ Gerade aufgelöst, und bezeichnen wir durch $\gamma_{h}$ die zu $m=1$ gehörigen Werthe der $c_{h}$, so folgt offenbar

$$
c_{h} \equiv \gamma_{h}^{m},
$$

wo nun die $\gamma$ von $m$ unabhängig sind. Es bleiben also nur die Grössen $\gamma$ noch zu bestimmen.

Diese Grössen bestimmt man aus dem Werthe $m=n-3$, indem man eine Reihe von Curven $(n-3)^{\text {ter }}$ Ordnung bildet, für welche die Argumente $u$ von vorn herein bekaunt sind. Erstlich nämlich kann man eine Curve $(n-3)^{\text {ter }}$ Ordnung durch sämmtliche Doppelpunkte legen. Die Argumente ihres Schnittpunktsystems sind

$$
\alpha_{1}, \quad \beta_{1}, \quad \alpha_{2}, \quad \beta_{2}, \ldots \frac{\alpha_{n, n-3}}{2}, \frac{\beta_{n, n-3}}{2} .
$$

Die der zweiten Gleichung (II.) entsprechenden Gleichungen werden dabei sämmtlich illusorisch, und es bleibt nur

$$
\text { (32.) } \Sigma\left(\alpha_{h}+\beta_{h}\right) \equiv 0 \text {. }
$$

Man erhält also hiedurch nur den Satz:

Die Summe der den Doppelpunkten entsprechenden Argumentenpaare ist gleich einem ganzen Vielfachen der Perioden.

Wenn man nun Curven $n-3^{\text {ter }}$ Ordnung legt, welche durch alle Doppelpunkte mit Ausschluss eines derselben $\left(\alpha_{h}, \beta_{h}\right)$ hindurchgehen, so hat man 
noch eine Bestimmung zu seiner Verfügung. Diese soll so getroffen werden, dass die zwei noch übrigen Schnittpunkte mit der Curve $n^{\text {ter }}$ Ordnung zusammenfallen. Es wird also eine Curve $(n-3)^{\text {ter }}$ Ordnung gesucht, welche durch $\frac{n . n-3}{2}-1$ Doppelpunkte geht, und die gegebene Curve berührt. Ist $u$ das Argument des Berührungspunktes, so giebt zunächst die erste der Gleichungen (II.) mit Rücksicht auf (32.)

also für $u$ die vier Werthe

$$
2 u \equiv \alpha_{h}+\beta_{h},
$$

$$
u=\frac{\alpha_{h}+\beta_{h}}{2}, \frac{\alpha_{h}+\beta_{h}}{2}+K, \frac{\alpha_{h}+\beta_{h}}{2}+i K^{\prime}, \frac{\alpha_{h}+\beta_{h}}{2}+K+i K^{\prime} .
$$

Es folgt daraus

$$
\left(\frac{H\left(\alpha_{h}-u\right)}{H\left(\beta_{h}-u\right)}\right)^{i}=1 \quad \text { oder }=e^{-i \pi \frac{\beta_{h}-\alpha}{K}}
$$

und die von den Gleichungen (II.) allein übrigbleibende Gleichung, welche dem Doppelpunkte $\alpha_{h}, \beta_{h}$ entspricht, liefert die Formel zur Bestimmung von $\gamma_{h}$ :

$$
\text { (33.) } \quad \gamma_{h}^{n-3} \equiv \dot{\Pi} \frac{\boldsymbol{H}\left(\boldsymbol{\alpha}_{h}-\boldsymbol{\alpha}_{i}\right) \boldsymbol{H}\left(\boldsymbol{\alpha}_{h}-\beta_{i}\right)}{\boldsymbol{H}\left(\boldsymbol{\beta}_{h}-\boldsymbol{\alpha}_{i}\right) \boldsymbol{H}\left(\boldsymbol{\beta}_{h}-\boldsymbol{\beta}_{i}\right)},
$$

wobei der über das Productzeichen gesetzte Punkt andeutet, dass dem Index $i$ alle Werthe von 1 bis $\frac{n . n-3}{2}$, mit Ausschluss des Index $h$ beigelegt werden sollen.

Hiedurch ist die Bestimmung der Constanten geleistet, und man kann das Resultat der Betrachtung in folgenden Satz ausdrücken:

Die Argumente $u$ des Schnittpunktsystemes einer Curve $m^{\text {ter }}$ Ordnung mit der gegebenen Curve mïssen den folgenden $\frac{n-1 . n-2}{2}$ Bedingungen genügen:

(III.)

$$
\left\{\begin{array}{c}
u_{1}+u_{2}+\cdots+u_{m n} \equiv 0, \\
\prod_{i=1}^{\longrightarrow} \frac{H\left(\alpha_{h}-u_{i}\right)}{H\left(\beta_{h}-u_{i}\right)} \equiv\left\{\dot{\Pi} \frac{H\left(\alpha_{h}-\alpha_{i}\right) H\left(\alpha_{h}-\beta_{i}\right)}{H\left(\beta_{h}-\alpha_{i}\right)}\right\}^{\frac{m}{n-3}}, \\
\left.h=1, \quad 2, \ldots \frac{n . n-3}{2} . \beta_{h}-\beta_{i}\right)
\end{array}\right.
$$

Man kann diesen Satz noch modificiren, indem man ihn für eine Curve ausspricht, welche durch einige der Doppelpunkte geht. Sind $\alpha_{1}, \beta_{1} ; \alpha_{2}$, $\beta_{2} ; \ldots \alpha_{\mu}, \beta_{\mu}$ die Parameter derjenigen Doppelpunkte, durch welche die Curve $m^{\text {ter }}$ Ordnung nicht gelegt ist, so wird von den aus der zweiten Gleichung (III.) herrührenden Bedingungen jede illusorisch, für welche $h>\mu$, und es bleibt folgender Satz: 
Clebsch, ebène Curven, deren Coord. elliptische Funct. eines Parameters sind. 233

Wenn eine Curve $m^{\text {ter }}$ Ordnung durch $\frac{n . n-3}{2}-\mu$ Doppelpunkte der Curve $n^{\text {ter }}$ Ordnung gelegt ist, und es sind $\alpha_{1}, \beta_{1} ; \alpha, \beta_{2} ; \ldots \alpha_{\mu}, \beta_{\mu}$ die Argumente der übrigen Doppelpunkte, so sind die noch übrigen $m n-n(n-3)+2 \mu$ Schnittpunkte beider Curven den folgenden $\mu+1 \mathrm{Be}-$ dingungen unterworfen:

$$
\begin{aligned}
& \left(\begin{array}{c}
u_{1}+u_{2}+\cdots+u_{k} \equiv \alpha_{1}+\beta_{1}+\alpha_{2}+\beta_{2}+\cdots+\alpha_{\mu}+\beta_{\mu}, \quad(k=m n-n(n-3)+2 \mu) \\
\prod_{i=1}^{i=k} \frac{H\left(\alpha_{h}-u_{i}\right)}{H\left(\beta_{h}-u_{i}\right)}
\end{array}\right. \\
& \equiv\left\{\prod_{i=1}^{i=\mu} \frac{H\left(\alpha_{h}-\alpha_{i}\right) H\left(\alpha_{h}-\beta_{i}\right)}{H\left(\beta_{h}-\alpha_{i}\right) H\left(\beta_{h}-\beta_{i}\right)}\right\}^{\frac{m}{n-3}} \cdot\left\{\prod_{i=\mu+1}^{i=\frac{n . n-3}{2}} \frac{H\left(\alpha_{h}-\alpha_{i}\right) H\left(\alpha_{h}-\beta_{i}\right)}{H\left(\beta_{h}-\alpha_{i}\right) H\left(\beta_{h}-\beta_{i}\right)}\right\}^{\frac{n-n+3}{n-3}}, \\
& h=1,2, \ldots \mu \text {. }
\end{aligned}
$$

§. 10

Umkehrungsproblem.

Ich gehe jetzt zu der Aufgabe über, aus einer gegebenen Anzahl von Schnittpunkten die übrigen zu bestimmen. Ist $m>n-3$, so bestimmen sich immer $\frac{n-1 . n-2}{2}$ durch die übrigen, sobald unter den gegebenen Punkten kein Doppelpunkt ist. Sind aber unter den gegebenen Punkten $\frac{n \cdot n-3}{2}-\mu$ Doppelpunkte, so bestimmen diese eine doppelt so grosse Anzahl von Argumenten, und es bleiben nur noch $\mu+1$ Schnittpunkte zu bestimmen übrig, so dass immer die Anzahl der aus den Gleichungen (III.), (IV.) bestimmten Schnittpunkte- die Anzahl der nicht dem System zugehörigen Doppelpunkte um Eins übertrifft. Denken wir uns also, um möglichste Allgemeinheit zu bewahren, das Schnittpunktsystem enthalte $\frac{n . n-3}{2}-\mu$ Doppelpunkte mit den Argumenten $\alpha_{\mu+1}, \beta_{\mu+1} ; \alpha_{\mu+2}, \beta_{\mu+2} ; \ldots$, und ausserdem noch $k-\mu-1$ $=m n-n(n-3)+\mu-1$ gegebene Punkte mit den Argumenten

$$
u_{\mu+2}, u_{\mu+3}, \ldots u_{k} .
$$

Die Argumente $u_{1}, u_{2}, \ldots u_{\mu+1}$ der noch übrigen Schnittpunkte sind dann nach (IV.) aus folgenden Gleichungen gegeben, in welchen alles Gegebene in die rechten Theile geworfen ist: 


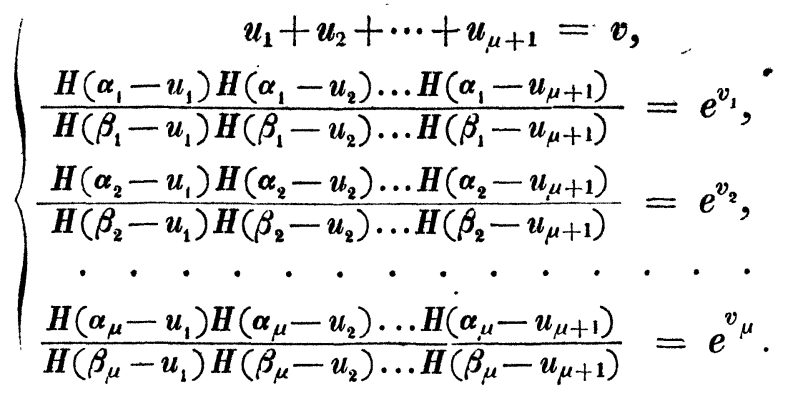

Die Aufgabe, aus diesen Gleichungen die Grössen

$$
z_{1}=\sin ^{2} \mathrm{am} u_{1}, \quad z_{2}=\sin ^{2} \mathrm{am} u_{2}, \ldots . \quad z_{\mu+1}=\sin ^{2} \mathrm{am} u_{\mu+1}
$$

mittels einer Gleichung $(\boldsymbol{\mu}+\mathbf{1})^{\text {ten }}$ Grades zu finden, umfasst (abgesehen von den speciellen Werthen der rechten Theile) ein Umkehrungsproblem von allgemeinem analytischen Interesse, insofern es aus dem Jacobischen Umkehrungsproblem für Abelsche Functionen hervorgeht, sobald die Abelschen Integrale durch Specialisirung ihrer Constanten in elliptische Integrale ausarten. Für $\mu=1$ ist ein besonderer Fall des Problems, auf einem von dem hier zu yerfolgenden abweichenden Wege, von Hrn. Rosenhain im ersten Capitel seiner Preisschrift über die vierfach periodischen Functionen gelöst worden.

Die Lösung des Umkehrungsproblems gelingt durch Anwendung des schon wiederholt benutzten Hermiteschen Satzes. Man setze zunächst

$$
u_{i}^{\prime}=u_{i}-\frac{v}{\mu+1}, \quad \alpha_{i}^{\prime}=\alpha_{i}-\frac{v}{\mu+1}, \quad \beta_{i}^{\prime}=\beta_{i}-\frac{v}{\mu+1}
$$

und drücke die $u$ durch die $u^{\prime}$ aus. Dadurch ändern sich die Gleichungen (V.) nur insofern, als überall die gestrichnen Buchstaben an Stelle der früheren treten, und als die erste Gleichung übergeht in .

$$
u_{1}^{\prime}+u_{2}^{\prime}+\cdots+u_{\mu+1}^{\prime}=0 \text {. }
$$

Dieses aber ist die Bedingung, unter welcher der Ausdruck

$$
\frac{H\left(\omega^{\prime}-u_{1}^{\prime}\right) H\left(\omega^{\prime}-u_{2}^{\prime}\right) \ldots H\left(\omega^{\prime}-u_{\mu+1}^{\prime}\right)}{\left(\Theta\left(\omega^{\prime}\right)\right)^{\mu+1}}
$$

nach dem erwähnten Satze in die Form einer ganzen rationalen Function von $\sqrt{ } z^{\prime}=\sin a m \omega^{\prime}$ und von $\sqrt{1-z^{\prime} .1-k^{2} z^{\prime}}$ gebracht werden kann, deren Coefficienten dann nur noch von den $u^{\prime}$ abhängen. Diese Function $\Phi\left(z^{\prime}\right)$ nimmt, wenn $\mu$ ungerade ist, die Form an

$$
\Phi\left(z^{\prime}\right)=F_{\frac{\mu+1}{2}}\left(z^{\prime}\right)+F_{\frac{\mu-3}{2}}\left(z^{\prime}\right) \cdot \sqrt{z^{\prime} \cdot 1-z^{\prime} \cdot 1-k^{2} z^{\prime}},
$$


wo die $F$ ganze Functionen ihres Arguments sind, deren Ordnung durch ihren Index angezeigt ist. Wenn aber $\mu$ gerade ist, so hat man

$$
\Phi\left(z^{\prime}\right)=F_{\frac{\mu}{2}}\left(z^{\prime}\right) \cdot \sqrt{z^{\prime}+F_{\frac{\mu-2}{2}}}\left(z^{\prime}\right) \cdot \sqrt{1-z^{\prime} \cdot 1-k^{2} z^{\prime}} ;
$$

in beiden Fällen enthält $\Phi$ noch $\mu+1$ unbestimmte Coefficienten.

Nach Einführung dieser Function $\Phi\left(z^{\prime}\right)$ nehmen nun die Gleichungen (V.) die Form an:

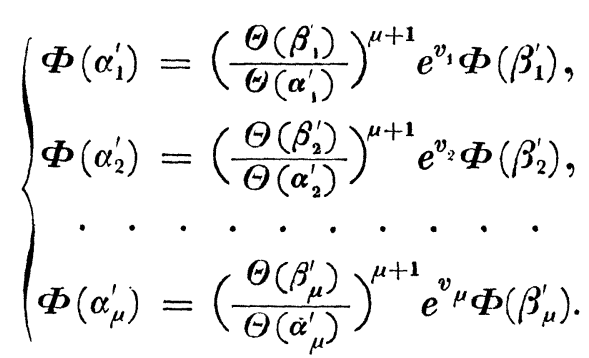

In diesen Gleichungen ist alles bekannt, bis auf die Coefficienten der Function $\Phi$, und man kann daher die Verhältnisse derselben aus den Gleichungen (34.) auf lineare Weise bestimmen, wozu die Anzahl derselben gerade hinreicht.

So ist nun $\boldsymbol{\Phi}\left(z^{\prime}\right)$ eine vollständig bekannte Function; wir wissen aber aus der ursprünglichen Entstehung derselben, dass sie für $\omega^{\prime}=u_{1}^{\prime}, u_{2}^{\prime}, \ldots u_{\mu+1}^{\prime}$ verschwindet. Die $\mu+1$ Wurzeln der aus $\Phi=0$ durch Quadriren entstehenden rationalen Gleichung in $z^{\prime}$ müssen also sein

$$
z_{1}^{\prime}=\sin ^{2} \mathrm{am} u_{1}^{\prime}, \quad z_{2}^{\prime}=\sin ^{2} \mathrm{am} u_{2}^{\prime}, \quad \ldots \quad z_{\mu+1}^{\prime}=\sin ^{2} \mathrm{am} u_{\mu+1}^{\prime},
$$

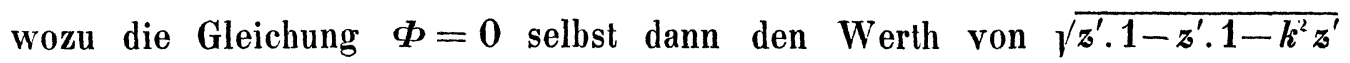
liefert, und so die $u$ vollständig bestimmt. Um die Gleichung $\boldsymbol{\Phi}=0$ aufzustellen, deren Wurzeln die Grössen (35.) sind, hat man also nur aus (34.) mit Hinzufügung von $\Phi\left(z^{\prime}\right)=0$ die unbekannten Coefficienten zu eliminiren, und die resultirende Gleichung rational zu machen. Hat man so die $z^{\prime}$ und die Werthe von $\sqrt{z^{\prime} .1-z^{\prime} \cdot 1-k^{2} z^{\prime}}$ gefunden, so liefert das Additionstheorem die Werthe der $z$ und der Ausdrücke $\sqrt{z .1-z .1-k^{2} z}$ ohne Weiteres.

Aus dem Gesagten geht hervor, dass man immer nur, und zwar mittels Auflösung einer Gleichung $\mu+1^{\text {ten }}$ Grades, ein einziges System oberer Grenzen angeben kann, welches die Gleichungen (V.) befriedigt.

\section{\$. 11.}

Andere Behandlung des Umkehrungsproblems.

Ich werde jetzt einige Formeln entwickeln, welche eine andere $\mathrm{Be}-$ handlungsweise des Problems gestatten. Zu diesen führt eine genauere Unter- 
suchung der aus den Gleichungen (34) und aus $\Phi\left(z^{\prime}\right)=0$ entspringenden Resultante, welche, gleich Null gesetzt, $z_{1}^{\prime}, z_{2}^{\prime}, \ldots z_{\mu+1}^{\prime}$ liefert. Dieselbe ist offenbar in Bezug auf die Grössen $\left(\frac{\Theta\left(\beta_{i}^{\prime}\right)}{\Theta\left(\alpha_{i}^{\prime}\right)}\right)^{\mu+1} e^{v_{i}}$ linear; und sie entsteht also aus der symbolischen Gleichung

$$
\left\{\begin{array}{r}
\left(\alpha_{1}^{\prime} \Theta\left(\alpha_{1}^{\prime}\right)^{\mu+1}-\beta_{1}^{\prime} \Theta\left(\beta_{1}^{\prime}\right)^{\mu+1} e^{v_{1}}\right)\left(\alpha_{2}^{\prime} \Theta\left(\alpha_{2}^{\prime}\right)^{\mu+1}-\beta_{2}^{\prime} \Theta\left(\beta_{2}^{\prime}\right)^{\mu+1} e^{v_{2}}\right) \ldots \\
\left(\alpha_{\mu}^{\prime} \Theta\left(\alpha_{\mu}^{\prime}\right)^{\mu+1}-\beta_{\mu}^{\prime} \Theta\left(\beta_{\mu}^{\prime}\right)^{\mu+1} e^{v_{\mu}}\right)=0 .
\end{array}\right.
$$

wenn man an Stelle der Producte der $\alpha, \beta$ passende Ausdrücke treten lässt. Diese Ausdrücke sind alle gleich gebildet, und es entspringen alle aus dem ersten, indem an Stelle der $\alpha$ successive mehrere der entsprechenden $\beta$ gesetzt werden. Bezeichnen wir demnach die Coefficienten von $1,-e^{v_{1}},-e^{v_{2}}$, $e^{v_{1}+v_{2}} \ldots$ durch

$$
\left(\omega^{\prime}, \alpha_{1}^{\prime}, \alpha_{2}^{\prime} \ldots \alpha_{\mu}^{\prime}\right),\left(\omega^{\prime}, \beta_{1}^{\prime}, \alpha_{2}^{\prime} \ldots \alpha_{\mu}^{\prime}\right),\left(\omega^{\prime}, \alpha_{1}^{\prime}, \beta_{2}^{\prime} \ldots \alpha_{\mu}^{\prime}\right),\left(\omega^{\prime}, \beta_{1}^{\prime}, \beta_{2}^{\prime} \ldots \alpha_{\mu}^{\prime}\right), \text { u. s. w., }
$$

so ist der erste derselben, der allein betrachtet zu werden braucht, die Resultante aus 、

$$
\Phi\left(\omega^{\prime}\right)=0, \quad \Phi\left(\alpha_{1}^{\prime}\right)=0, \quad \Phi\left(\alpha_{2}^{\prime}\right)=0, \quad \Phi\left(\alpha_{\mu}^{\prime}\right)=0,
$$

also eine alternirende Function der $\mu+1$ Argumente.

Nach dem Hermiteschen Satz aber kann diese Resultante ersetzt werden durch

$$
C \cdot \frac{H\left(\omega^{\prime}-\alpha_{1}^{\prime}\right) H\left(\omega^{\prime}-\alpha_{2}^{\prime}\right) \ldots H\left(\omega^{\prime}-\alpha_{\mu}^{\prime}\right) \cdot H\left(\omega^{\prime}+\alpha_{1}^{\prime}+\alpha_{2}^{\prime}+\cdots+\alpha_{\mu}^{\prime}\right)}{\Theta^{\mu+1}\left(\omega^{\prime}\right)},
$$

wo $C$ von $\omega^{\prime}$ nicht mehr abhängt. Da nun durch Vertauschung von $\omega^{\prime}$ mit einem der $\alpha^{\prime}$ die Function nur ihr Zeichen wechselt, so kann man setzen

$$
\left(\omega^{\prime}, \alpha_{1}^{\prime}, \alpha_{2}^{\prime} \ldots \alpha_{\mu}^{\prime}\right)=\frac{H\left(\omega^{\prime}+\alpha_{1}^{\prime}+\alpha_{2}^{\prime}+\cdots+\alpha_{\mu}^{\prime}\right) \cdot \Delta\left(\omega^{\prime}, \alpha_{1}^{\prime}, \alpha_{2}^{\prime}, \ldots \alpha_{\mu}^{\prime}\right)}{\left\{\Theta\left(\omega^{\prime}\right) \Theta\left(\alpha_{1}^{\prime}\right) \Theta\left(\alpha_{2}^{\prime}\right) \ldots \Theta\left(\alpha_{\mu}^{\prime}\right)\right\}^{\mu+1}}
$$

wobei $\boldsymbol{\Delta}$ das Product der $\boldsymbol{H}$ aller Differenzen der Argumente bedeutet, die Differenzen so gebildet, dass immer ein folgendes Argument von einem vorangehenden abgezogen wird.

Ersetzen wir in diesen Ausdrücken die $\omega^{\prime}, \alpha^{\prime} \ldots$ wieder durch die $\omega-\frac{v}{\mu+1}, \alpha-\frac{v}{\mu+1}, \ldots$, so kann man an Stelle von (36.) die symbolische Gleichung

$$
\text { (38.) } \quad \Omega(\omega)=\prod_{i=1}^{i=\mu}\left(\alpha_{i}-e^{v_{i}} \beta_{i}\right)=0
$$


treten lassen, und muss dann die Producte der $\alpha, \beta$ ersetzen durch Ausdrücke von der Form

$$
\text { (39.) }\left[\omega, \alpha_{1}, \alpha_{2}, \ldots \alpha_{\mu}\right]=H\left(\omega+\alpha_{1}+\alpha_{2}+\cdots+\alpha_{\mu}-v\right) . \Delta\left(\omega, \alpha_{1}, \alpha_{2}, \ldots \alpha_{\mu}\right) \text {. }
$$

Dabei ist nur im Nenner der Factor $\Theta\left(\omega-\frac{v}{\mu+1}\right)^{\mu-1}$ entfernt, welcher allen Ausdrücken gemeinschaftlich ist, und die übrigen Factoren des Nenners sind gegen die in dem früheren symbolischen Producte auftretenden Potenzen der $\Theta$ aufgehoben.

Da nun, abermals nach dem Hermiteschen Satz,

$$
\frac{\boldsymbol{\Omega}(\boldsymbol{\omega})}{\Theta\left(\boldsymbol{\omega}^{\prime}\right)^{\mu+1}}=K \cdot \frac{H\left(\omega^{\prime}-u_{1}^{\prime}\right) H\left(\omega^{\prime}-u_{2}^{\prime}\right) \ldots H\left(\omega^{\prime}-u_{\mu+1}^{\prime}\right)}{\Theta\left(\omega^{\prime}\right)^{\mu+1}}
$$

so hat man auch

$$
\Omega(\omega)=K \cdot H\left(\omega-u_{1}\right) \cdot H\left(\omega-u_{2}\right) \ldots H\left(\omega-u_{\mu+1}\right),
$$

und dabei ist $K$ von $\omega$ unabhängig. Um zunächst diese Grösse fortzuschaffen, dividire ich diese Gleichung durch diejenige, welche durch Vertauschung von $\omega$ mit einer beliebigen andern Grösse $\omega_{0}$ entsteht, und erhalte:

$$
\text { (40.) } \frac{\Omega(\omega)}{\Omega\left(\omega_{0}\right)}=\frac{H\left(\omega-u_{1}\right) H\left(\omega-u_{2}\right) \ldots H\left(\omega-u_{\mu+1}\right)}{H\left(\omega_{0}-u_{1}\right) H\left(\omega_{0}-u_{2}\right) \ldots H\left(\omega_{0}-u_{\mu+1}\right)} \text {. }
$$

Aus dieser Formel entspringt eine Reihe von bemerkenswerthen Gleichungen. Ersetzt man zunächst $\omega$ und $\omega_{0}$ durch die Werthe $0, K, i K^{\prime}, K+i K_{1}^{\prime}$ so erhält man:

(41.)

$$
\begin{aligned}
& \sqrt{\sqrt{z_{1} \cdot z_{2} \ldots z_{\mu+1}}} \quad=\prod_{i=1}^{i=\mu+1} \sin \mathrm{am} u_{i} \\
& =\frac{1}{(\sqrt{-1} \cdot \sqrt{k} \cdot \sqrt[4]{q})^{\mu+1}} \cdot e^{\frac{i \pi v}{2 K}} \cdot \frac{\Omega(0)}{\Omega\left(i K^{\prime}\right)}, \\
& \sqrt{1-z_{1} .1-z_{2} \ldots 1-z_{\mu+1}} \quad=\prod_{i=1}^{i=\mu+1} \cos a m u_{i} \\
& =\left(\frac{\sqrt{-1} \cdot \sqrt{k^{\prime}}}{\sqrt{k} \cdot \sqrt[4]{q}}\right)^{\mu+1} \cdot e^{\frac{i \pi v}{2 K}} \cdot \frac{\Omega(K)}{\Omega\left(i K^{\prime}\right)}, \\
& \sqrt{1-k^{2} z_{1} \cdot 1-k^{2} z_{2} \ldots 1-k^{2} z_{\mu+1}}=\prod_{i=1}^{i=\mu+1} \Delta \operatorname{am} u_{i} \\
& =\left(\sqrt{-1} \cdot \sqrt{k^{\prime}}\right)^{\mu+1} \cdot e^{\frac{i \pi v}{2 K}} \cdot \frac{\Omega\left(K+i K^{\prime}\right)}{\Omega\left(i K^{\prime}\right)} .
\end{aligned}
$$

Lassen wir ferner in (40.) $\omega$ in $-\omega, \omega_{0}$ in $-\omega_{0}$ übergehen, und be- 
238 Clebsch, ebene Curven, derè Coord. elliptische Funct. eines Parameters sind.

merken, dass

$$
\frac{H(\omega-u) \boldsymbol{H}(\omega+u)}{H\left(\omega_{0}-u\right) H\left(\omega_{0}+u\right)}=\frac{\Theta^{2}(\omega)}{\Theta^{2}\left(\omega_{0}\right)} \cdot \frac{\sin ^{2} \mathrm{am} \omega-\sin ^{2} \mathrm{am} u}{\sin ^{2} a \mathrm{am} \omega_{0}-\sin ^{2} \mathrm{am} u},
$$

so finden wir:

$$
\frac{z-z_{1} \cdot z-z_{2} \ldots z-z_{\mu+1}}{z_{0}-z_{1} \cdot z_{0}-z_{2} \ldots z_{0}-z_{\mu+1}}=\frac{\Omega(\omega) \cdot \Omega(-\omega)}{\Omega\left(\omega_{0}\right) \cdot \Omega\left(-\omega_{0}\right)} \cdot\left(\frac{\Theta\left(\omega_{0}\right)}{\Theta(\omega)}\right)^{2 \mu+2}
$$

wo

$$
\bar{z}=\sin ^{2} \operatorname{am} \omega, \quad z_{0}=\sin ^{2} a m \omega_{0} .
$$

Wenn wir in dieser Formel für $z, z_{0}$ eine Reihe specieller Werthe setzen, so erhalten wir lineare Gleichungen zur Bestimmung der symmetrischen Functionen von $z_{1}, z_{2}, \ldots z_{\mu+1}$, und wir sind auf diese Weise im Stande, direct eine Gleichung aufzustellen, deren Wurzeln die Grössen $z$ sind; eine neue Art, das oben ausgeführte Umkehrungsproblem zu behandeln.

Die Gleichung (42.) liefert eine einfachere Form der rechten Seite, sobald man für $z, z_{0}$ ein Parameterpaar $a, b$ eines der $\mu$ Doppelpunkte, und also für $\omega, \omega_{0}$ ein Paar der Reihe $\alpha_{1}, \beta_{1} ; \alpha_{2}, \beta_{2} ; \ldots \alpha_{\mu}, \beta_{\mu}$ wählt. Alsdann ist wegen (40.) $\frac{\Omega\left(\alpha_{i}\right)}{\Omega\left(\beta_{i}\right)}=e^{v_{i}}$, und man hat demnach $\mu$ Gleichungen der folgenden Art:

$$
\frac{a_{i}-z_{1} \cdot a_{i}-z_{2} \ldots a_{i}-z_{\mu+1}}{b_{i}-z_{1} \cdot b_{i}-z_{2} \ldots b_{i}-z_{\mu+1}}=e^{v_{i}} \cdot \frac{\Omega\left(-\alpha_{i}\right)}{\Omega\left(-\beta_{i}\right)} \cdot\left(\frac{\Theta\left(\beta_{i}\right)}{\Theta\left(\alpha_{i}\right)}\right)^{2 \mu+2} \cdot
$$

Die angeführten Formeln des Hrn. Rosenhain gehen aus den hier gegebenen hervor, wenn $\operatorname{man} \mu=1$ und $\beta=-\alpha$ setzt, durch welchen letztern Umstand einige specielle Vereinfachungen eintreten.

\section{§. 12.}

Periodicität.

In dem soeben betrachteten Umkehrungsproblem sind die Grössen $z_{1}, z_{2}, \ldots z_{\mu+1}$ oder ihre symmetrischen Functionen periodische Functionen von $v, v_{1}, v_{2}, \ldots v_{\mu}$, und zwar $(\mu+2)$ fach periodische. Die eine Classe der Perioden umfasst $\mu$ derselben, und rührt von den Exponentialgrössen $e^{v_{i}}$ her. Die $z$ bleiben unverändert, wenn man irgend eine der $v_{i}$ um $2 h_{i} \pi \sqrt{-1}$ vermehrt. Dagegen rühren die andern beiden Perioden von den elliptischen Integralen her. Vermehrt man $v$ um $2 n K+2 n i K^{\prime}$, so können dabei sämmtliche z ungeändert bleiben, vorausgesetzt nur, dass man gleichzeitig die $v_{i}$.gehörig 
ändert. Geht nämlich $u_{k}$ in $u_{k}+2 m K+2 n i K^{\prime}$ über, so verwandelt sich $\frac{\boldsymbol{H}\left(\boldsymbol{\alpha}_{i}-\boldsymbol{u}_{k}\right)}{\boldsymbol{H}\left(\boldsymbol{\beta}_{i}-\boldsymbol{u}_{k}\right)}$ in

$$
e^{\frac{n \pi V \overline{-1}}{K}\left(\alpha_{i}-\beta_{i}\right)} \frac{H\left(\alpha_{i}-u_{k}\right)}{H\left(\beta_{i}-u_{k}\right)}
$$

Erhalten also alle $u$ ähnliche Zuwächse, und bedeutet jetzt $m$ die Summe aller $m, n$ die Summe aller $n$, so geht $v_{i}$ in $v_{i}+\frac{n \pi \sqrt{-1}}{K}\left(\alpha_{i}-\beta_{i}\right)$ über. Gehört also ein gewisses System

zu den Variabeln

$$
\mathbf{z}_{1}, \quad \mathbf{z}_{2}, \ldots \mathbf{z}_{\mu+1},
$$

$$
v, v_{1}, \ldots v_{\mu},
$$

so ist das allgemeinste System von Variabeln, welchem dieselben Grössen z angehören, folgendes:

$$
\left\{\begin{array}{l}
v^{\prime}=v+2 m K+2 n i K^{\prime}, \\
v_{1}^{\prime}=v_{1}+2 h_{1} i \pi+\frac{n i \pi}{K}\left(\alpha_{1}-\beta_{1}\right), \\
v_{2}^{\prime}=v_{2}+2 h_{2} i \pi+\frac{n i \pi}{K}\left(\alpha_{2}-\beta_{2}\right), \\
\cdot \cdot \cdot \cdot \cdot \cdot \cdot \cdot \cdot \cdot \cdot \cdot \\
v_{\mu}^{\prime}=v_{\mu}+2 h_{\mu} i \pi+\frac{n i \pi}{K}\left(\alpha_{\mu}-\beta_{\mu}\right),
\end{array}\right.
$$

wobei die $m, n, h$ von einander unabhängige, willkürliche ganze Zahlen bedeuten.

\section{\$. 13.}

Ein specieller Fall des Umkehrungsproblems.

Es soll jetzt die Frage untersucht werden, welche Bedingung zwischen den Grössen $v, v_{1}, \ldots v_{\mu}$ eintreten muss, damit dieselbe gleich ähnlichen Ausdrücken wie (V.) werden, nur mit einer Unbekannten $u$ weniger; damit also Gleichungen folgender Art bestehen:

$$
\left\{\begin{array}{l}
u_{1}+u_{2}+\cdots+u_{\mu}=v, \\
\frac{H\left(\alpha_{1}-u_{1}\right) H\left(\alpha_{1}-u_{2}\right) \ldots H\left(\alpha_{1}-u_{\mu}\right)}{\bar{H}\left(\beta_{1}-u_{1}\right) H\left(\beta_{1}-u_{2}\right) \ldots H\left(\beta_{1}-u_{\mu}\right)}=e^{v_{1}}, \\
\frac{H\left(\alpha_{2}-u_{1}\right) H\left(\alpha_{2}-u_{2}\right) \ldots H\left(\alpha_{2}-u_{\mu}\right)}{H\left(\beta_{2}-u_{1}\right) H\left(\beta_{2}-u_{2}\right) \ldots H\left(\beta_{2}-u_{\mu}\right)}=e^{v_{2}}, \\
\cdot \cdot \cdot \cdot \cdot \cdot \cdot \cdot \cdot \cdot \cdot \cdot \cdot \cdot \cdot \\
\frac{H\left(\alpha_{\mu}-u_{1}\right) H\left(\alpha_{\mu}-u_{2}\right) \ldots H\left(\alpha_{\mu}-u_{\mu}\right)}{H\left(\beta_{\mu}-u_{1}\right) H\left(\beta_{\mu}-u_{2}\right) \ldots H\left(\beta_{\mu}-u_{\mu}\right)}=e^{v_{\mu}} .
\end{array}\right.
$$


Dieser Fall lässt sich ganz nach Art des oben gelösten Umkehrungsproblemes behandeln; ja man kann die oben entwickelten Formeln hier wiederum benutzen. Man hat nur die Function $\Phi$ hier durch eine andere zu ersetzen, welche nur für $\mu$ Werthe verschwindet, und sodann aus einem System von Gleichungen wie (34.) zu eliminiren.

Setzt man also

$$
\left[\alpha_{1}, \alpha_{2}, \ldots \alpha_{\mu}\right]=\boldsymbol{H}\left(\alpha_{1}+\alpha_{2}+\cdots+\alpha_{\mu}-v\right) \cdot \Delta\left(\alpha_{1}, \alpha_{2}, \ldots \alpha_{\mu}\right),
$$

und bildet man eine Reihe ähnlicher Ausdrücke, indem man an Stelle der $\alpha$ allmälig die $\beta$ einführt; ersetzt man endlich diese Ausdrücke symbolisch durch die Producte der $\alpha$ und $\beta$, so ist die gesuchte Eliminationsgleichung in der symbolischen Formel enthalten:

$$
\text { (46.) } \quad\left(\alpha_{1}-e^{v_{1}} \beta_{1}\right)\left(\alpha_{2}-e^{v_{2}} \beta_{2}\right) \ldots\left(\alpha_{\mu}-e^{v_{\mu}} \beta_{\mu}\right)=0 \text {. }
$$

Ich werde jetzt einen besonderen Fall untersuchen, in welchem diese Gleichung erfüllt sein kann. In diesem Falle ist

$$
\left\{\begin{array}{l}
v=\frac{1}{2} \sum_{1}^{\mu}\left(\alpha_{h}+\beta_{h}\right)+p K+q i K^{\prime}, \\
e^{v_{i}}=(-1)^{h_{i}} \sqrt{\prod_{k=1}^{k=\mu} \frac{H\left(\alpha_{h}-\alpha_{k}\right)}{H\left(\beta_{h}-\alpha_{k}\right)} \frac{H\left(\alpha_{h}-\beta_{k}\right)}{\boldsymbol{H}\left(\beta_{h}-\beta_{k}\right)} e^{\frac{q \pi i\left(\alpha_{i}-\beta_{i}\right)}{2 K}} .} .
\end{array}\right.
$$

Die Quadratwurzeln sollen so genommen werden, dass, wenn

$$
e^{v_{1}+v_{2}+\ldots+v_{\mu}}=(-1)^{h_{1}+h_{2}+\ldots+h_{\mu}} \cdot M \cdot e^{\frac{q \pi \sqrt{-1}}{2 K}\left(\left(\alpha_{1}-\beta_{1}\right)+\left(\alpha_{2}-\beta_{2}\right) \ldots\right)}
$$

gesetzt wird,

$$
\text { (49.) } \quad \dot{M}=\frac{\Delta\left(\alpha_{1}, \alpha_{2}, \ldots \alpha_{\mu}\right)}{\Delta\left(\beta_{1}, \beta_{2}, \ldots \beta_{\mu}\right)} \text {, }
$$

was durch passende Wahl eines einzigen Vorzeichens ermöglicht wird.

Die geometrische Bedeutung dieses Falles wird sofort deutlich, wenn man die Werthe von $v$ und $e^{v_{i}}$ in die Gleichungen (45.) einführt, und sodann die erste Gleichung mit 2 multiplicirt, die andern quadrirt. Mit Hinweglassung von Vielfachen der Perioden erhält man dann:

$$
\begin{gathered}
2\left(u_{1}+u_{2}+\cdots+u_{\mu}\right) \equiv 0 \\
\left\{\prod_{i=1}^{i=\mu} \frac{H\left(\alpha_{h}-u_{i}\right)}{H\left(\beta_{h}-u_{i}\right)}\right\}^{2} \equiv \prod_{k=1}^{\dot{\mu}} \frac{H\left(\alpha_{h}-\alpha_{k}\right)}{H\left(\beta_{h}-\alpha_{k}\right)} \frac{H\left(\alpha_{h}-\beta_{k}\right)}{H\left(\beta_{h}-\beta_{k}\right)}, \\
h=1, \quad 2, \ldots . \mu .
\end{gathered}
$$

Dies sind die Gleichungen (IV.) für $m=n-3$, wenn man die Argumente paar- 
weise zusammenfallen lässt, und sie stellen also die Bedingungen dar für die Argumente einer Curve $(n-3)^{\text {ter }}$ Ordnung, welche durch $\frac{n . n-3}{2}-\mu$ Doppelpunkte geht, und die Curve vierter Ordnung noch in $\mu$ verschiedenen Punkten berührt. Es zeigt sich, dass solche Curven möglich sind, dass aber zwischen den ganzen Zahlen $p, q, h_{1}, \ldots h_{\mu}$ eine Bedingung stattfindet.

Betrachten wir das Aggregat zweier Terme von (46.) deren einer etwa in $e^{v_{1}+v_{2}+\ldots+v_{r}}$ multiplicirt ist, während der andere durch den Factor

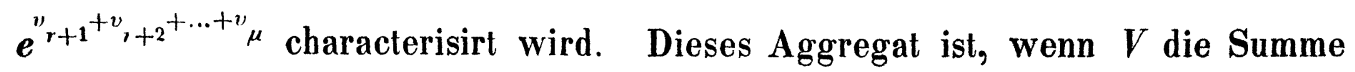
$v_{1}, v_{2}, \ldots v_{\mu}$ bezeichnet:

$$
\left(\left[\beta_{1}, \beta_{2}, \ldots \beta_{r}, \alpha_{r+1}, \ldots \alpha_{\mu}\right] e^{\nu_{1}+v_{2}+\ldots+v_{r}}+(-1)^{\mu}\left[\alpha_{1}, \alpha_{2}, \ldots \alpha_{r}, \beta_{r+1}, \ldots \beta_{\mu}\right] e^{\nu-v_{1}-v_{2}-\ldots-v_{r}}\right) .
$$

Ich dividire durch

$$
\left[\alpha_{1}, \alpha_{2}, \ldots \alpha_{r}, \beta_{r+1}, \ldots \beta_{\mu}\right] e^{-v_{1}-v_{2}-\ldots-v_{r}}
$$

und betrachte den Ausdruck:

$$
\text { (50.) } \pm\left(\frac{\left[\beta_{1}, \beta_{2}, \ldots \beta_{r}, \alpha_{r+1}, \ldots \alpha_{\mu}\right]}{\left[\alpha_{1}, \alpha_{2}, \ldots \alpha_{r}, \beta_{r+1}, \ldots \beta_{\mu}\right]} \cdot e^{2\left(v_{1}+v_{2}+\ldots+v_{r}\right)}+(-1)^{\mu} e^{\nu}\right) .
$$

Nach der Definition der eingeklammerten Ausdrücke hat man nun für das erste Glied der Klammer:

$$
\begin{gathered}
\frac{H\left(\beta_{1}+\beta_{2}+\cdots+\beta_{r}+\alpha_{r+1}+\cdots+\alpha_{\mu}-v\right)}{H\left(\alpha_{1}+\alpha_{2}+\cdots+\alpha_{r}+\beta_{r+1}+\cdots+\beta_{\mu}-v\right)} \cdot \frac{\Delta\left(\beta_{1}, \beta_{2}, \ldots \beta_{r}, \alpha_{r+1}, \ldots \alpha_{\mu}\right)}{\Delta\left(\alpha_{1}, \alpha_{2}, \ldots \alpha_{r}, \beta_{r+1}, \ldots \beta_{\mu}\right)} \\
\qquad \prod_{h=1}^{h=r} \prod_{i=1}^{i=\mu} \frac{H\left(\alpha_{h}-\alpha_{i}\right) H\left(\alpha_{h}-\beta_{i}\right)}{H\left(\beta_{h}-\alpha_{i}\right) H\left(\beta_{h}-\beta_{i}\right)} \cdot \varrho,
\end{gathered}
$$

wo

Ist nun

$$
\varrho=e^{\frac{q \pi V-1}{K}\left(\left(\alpha_{1}-\beta_{1}\right)+\left(\alpha_{2}-\beta_{2}\right)+\ldots+\left(\alpha_{r}-\beta_{r}\right)\right)} .
$$

$$
\sigma=\beta_{1}+\beta_{2}+\cdots+\beta_{r}+\alpha_{r+1}+\cdots+\alpha_{\mu}-\frac{1}{2} \sum_{1}^{\mu}\left(\alpha_{h}+\beta_{h}\right)
$$

so ist auch

$$
-\sigma=\alpha_{1}+\alpha_{2}+\cdots+\alpha_{r}+\beta_{r+1}+\cdots+\beta_{\mu}-\frac{1}{2} \sum_{1}^{\mu}\left(\alpha_{h}+\beta_{h}\right) .
$$

Bezeichnet ferner immer $i, i^{\prime}$ einen der Indices $1,2, \ldots r, k, k^{\prime}$ einen der Indices $r+1, r+2, \ldots \frac{n . n-3}{2}$, und sind die $\Pi$ fortan Zeichen von Differenzproducten, so kann man statt obigen Ausdrucks folgenden setzen: 


$$
\begin{gathered}
-\rho \frac{H\left(\sigma-p K-q i K^{\prime}\right)}{H\left(\sigma+p K+q i K^{\prime}\right)} \frac{\dot{\Pi} H\left(\beta_{i}-\beta_{i^{\prime}}\right) \cdot \Pi H\left(\beta_{i}-\alpha_{k}\right) \cdot \dot{\Pi} H\left(\alpha_{k}-\alpha_{k^{\prime}}\right)}{\dot{\Pi} H\left(\alpha_{i}-\alpha_{i^{\prime}}\right) \cdot \Pi H\left(\alpha_{i}-\beta_{k}\right) \cdot \dot{\Pi} H\left(\beta_{k}-\beta_{k^{\prime}}\right)} \\
\cdot \frac{\left\{\dot{\Pi} H\left(\alpha_{i}-\alpha_{i^{\prime}}\right)\right\}^{2} \Pi H\left(\alpha_{i}-\alpha_{k}\right) \dot{\Pi} H\left(\alpha_{i}-\beta_{i^{\prime}}\right) \Pi H\left(\alpha_{i}-\beta_{k}\right)}{\left\{\dot{\Pi} H\left(\beta_{i}-\beta_{i^{\prime}}\right)\right\}^{2} \Pi H\left(\beta_{i}-\beta_{k}\right) \dot{\Pi} H\left(\beta_{i}-\alpha_{i^{\prime}}\right) \Pi H\left(\beta_{i}-\alpha_{k}\right)}=-\rho \cdot \frac{H\left(\sigma-p K-q i K^{\prime}\right)}{H\left(\sigma+p K+q i K^{\prime}\right)} \cdot M .
\end{gathered}
$$

Führt man dies und den Werth von e ein, so enthält der Ausdruck (50.) den Factor

$$
-\varrho \frac{\boldsymbol{H}\left(\sigma-p \boldsymbol{K}-\boldsymbol{q i \boldsymbol { K } ^ { \prime } )}\right.}{\boldsymbol{H}\left(\sigma+p \boldsymbol{T}+\boldsymbol{q i \boldsymbol { K } ^ { \prime } )}\right.}+(-1)^{h_{1}+h_{2}+\ldots+h_{\mu}+\mu} \cdot e^{\frac{q \pi v-1}{2 K}\left(\alpha_{1}-\beta_{1}+\alpha_{2}-\beta_{2}+\ldots+\alpha_{\mu}-\beta_{\mu}\right)}
$$

und er verschwindet also, wenn dieser Factor verschwindet; ja die ganze Gleichung (46.) ist erfüllt, wenn alle Factoren dieser Art gleichzeitig zum Verschwinden gebracht werden können. Dies hängt von den Zahlen $p, q$ ab; man hat

$$
\frac{\boldsymbol{H}\left(\boldsymbol{\sigma}-\boldsymbol{p} \boldsymbol{K}-\boldsymbol{q} i \boldsymbol{K}^{\prime}\right)}{\boldsymbol{H}\left(\boldsymbol{\sigma}+\boldsymbol{p} \overline{\boldsymbol{K}}+q i \boldsymbol{K}^{\prime}\right)}=(-1)^{p} \frac{\boldsymbol{H}\left(\boldsymbol{\sigma}+\boldsymbol{p} \boldsymbol{K}-q i \boldsymbol{K}^{\prime}\right)}{\boldsymbol{H}\left(\boldsymbol{\sigma}+\boldsymbol{p} \overline{\boldsymbol{K}}+q i \boldsymbol{K}^{\prime}\right)}=(-1)^{q+p} e^{\frac{\pi q(\sigma+p K) V-1}{K}} .
$$

Wenn man nun diesen Werth in den obigen Ausdruck einführt, und die Werthe von $\rho, \sigma$ substituirt, so bleibt nur die Gleichung übrig:

$$
(-1)^{(q+1)(p+1)+h_{1}+h_{2}+\ldots+h \mu}=(-1)^{\mu+1} \text {, }
$$

und man hat den Satz:

Die Gleichungen:

$$
\begin{gathered}
u_{1}+u_{2}+\cdots+u_{\mu}=p \boldsymbol{K}+q i \boldsymbol{K}^{\prime}+\frac{1}{2} \sum_{1}^{\mu}\left(\alpha_{k}+\beta_{h}\right), \\
\frac{H\left(\alpha_{k}-u_{1}\right) H\left(\alpha_{k}-u_{2}\right) \ldots H\left(\alpha_{k}-u_{\mu}\right)}{H\left(\beta_{k}-u_{1}\right) H\left(\beta_{k}-u_{2}\right) \ldots H\left(\beta_{k}-u_{\mu}\right)}=(-1)^{h k} e^{\frac{q \pi i\left(\alpha_{k}-\beta_{k}\right)}{2 K}} \cdot \sqrt{\prod_{i=1}^{i=\mu} \frac{H\left(\alpha_{k}-\alpha_{i}\right) H\left(\alpha_{k}-\beta_{i}\right)}{H\left(\beta_{k}-\alpha_{i}\right) H\left(\beta_{k}-\beta_{i}\right)}} \\
k=1,2, \ldots, \mu,
\end{gathered}
$$

in denen $p, q, h_{k}$ ganze Zahlen bedeuten, und wo die Vorzeichen der Quadratwurzeln so zu wählen sind, dass das Product aller gleich

$$
\frac{\Delta\left(\alpha_{1}, \alpha_{2}, \ldots \alpha_{\mu}\right)}{\Delta\left(\beta_{1}, \beta_{2}, \ldots \beta_{\mu}\right)}
$$

ist, können immer zusammen bestehen, sobald

$$
(q+1)(p+1)+h_{1}+h_{2}+\cdots+h_{\mu} \equiv \mu+1 \quad(\bmod .2)
$$

Die zu den $u$ gehörigen $z$ zu finden, wenn die Gleichungen (45.) zusammen 
bestehen können, hat keine Schwierigkeit: man lässt eine der Gleichungen aus, und hat das früher behandelte Problem vor sich.

\section{\$. 14.}

Einfluss von Rückkehrpunkten.

Wenn von den $\frac{n . n-3}{2}$ Doppelpunkten der vorliegenden Curve $n^{\text {ter }}$ Ordnung einige in Rückkehrpunkte übergehen, so treten in den Betrachtungen der vorigen Paragraphen nur wenige Modificationen ein. Für einen solchen Punkt wird dann $\beta=\alpha+\varepsilon$, und $\varepsilon$ convergirt gegen Null. In dem Abelschen Theorem (II. oder III.) tritt daher an Stelle des Quotienten $H\left(\alpha_{i}-u\right): H\left(\beta_{i}-u\right)$ der Ausdruck

$$
\frac{H\left(\alpha_{i}-u\right)}{H\left(\alpha_{i}+\varepsilon-u\right)}=1-\varepsilon \frac{H^{\prime}\left(\alpha_{i}-u\right)}{H\left(\alpha_{i}-u\right)},
$$

und die betreffende Gleichung des Abelschen Theorems wird

$$
\frac{H^{\prime}\left(\alpha_{i}-u_{1}\right)}{H\left(\alpha_{i}-u_{1}\right)}+\frac{H^{\prime}\left(\alpha_{i}-u_{2}\right)}{H\left(\alpha_{i}-u_{2}\right)}+\cdots+\frac{H^{\prime}\left(\alpha_{i}-u_{m n}\right)}{H\left(\alpha_{i}-u_{m n}\right)}=\text { Const., }
$$

wo der Werth der Constante aus dem der früheren Constante durch einen Grenzübergang leicht zu ermitteln ist. Aber auch in dem Umkehrungsproblem (V.) tritt an Stelle der betreffenden Gleichung eine andre; man muss

setzen, und erhält:

$$
v_{i}=-\varepsilon \zeta_{i}
$$

$$
\frac{H^{\prime}\left(\alpha_{i}-u_{1}\right)}{H\left(\alpha_{i}-u_{1}\right)}+\frac{H^{\prime}\left(\alpha_{i}-u_{2}\right)}{H\left(\alpha_{i}-u_{2}\right)}+\cdots+\frac{H^{\prime}\left(\alpha_{i}-u_{\mu+1}\right)}{H\left(\alpha_{i}-u_{\mu+1}\right)}=\zeta_{i} .
$$

Das Resultat des Umkehrungsproblems ist aus dem früheren noch immer durch einen Grenzübergang leicht abzuleiten; aber es ist wichtig, dass durch das Eintreten dieses Rückkehrpunktes die eine Periode ausfällt, nämlich diejenige, welche von der Exponentialgrösse $e^{v^{i}}$ herrührte. Bei $\varkappa$ Rückkehrpunkten ist die Anzahl der Perioden also nur noch $\frac{n . n-3}{2}+2-\varkappa$; und wenn eine Anzahl von Doppelpunkten bei dem Umkehrungsproblem ausgeschlossen wird, so dass nur noch $\mu$ übrig bleiben, unter denen sich $\varkappa^{\prime}$ Rückkehrpunkte befinden, so reducirt jene Zahl sich auf $\mu+2-\varkappa^{\prime}$.

Eine obere Grenze für die Anzahl von Doppelpunkten, welche in Rückkehrpunkte übergehen können, liefert die Zahl der Wendepunkte der Curve, welche $3 n-2 x$ ist. Die Anzahl der Rückkehrpunkte kann also $\frac{3 n}{2}$ nicht überschreiten. 
244 Clebsch, ebene Curven, deren Coord. elliptische Funct. eines Parameters sind.

\section{S. 15. \\ Geometrische Sätze.}

Für die geometrischen Anwendungen, zu denen ich mich jetzt wende, ist es etwas bequemer, statt der im Abelschen Theorem auftretenden Grössen

$$
\frac{H\left(\boldsymbol{\alpha}_{i}-u\right)}{\boldsymbol{H}\left(\boldsymbol{\beta}_{i}-u\right)}
$$

solche einzuführen, welche sich von den Logarithmen dieser Grössen nur um passend gewählte Constanten unterscheiden. Ich setze

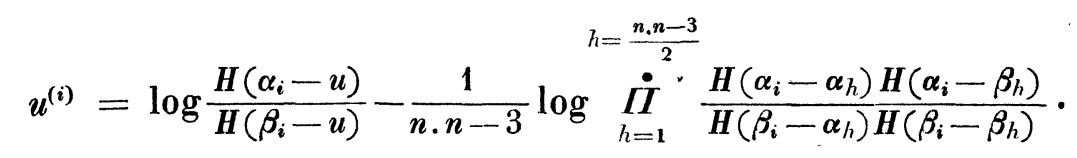

Die Gleichungen für ein Schnittpunktsystem (II. oder III.) gehen dann in die einfache Form über

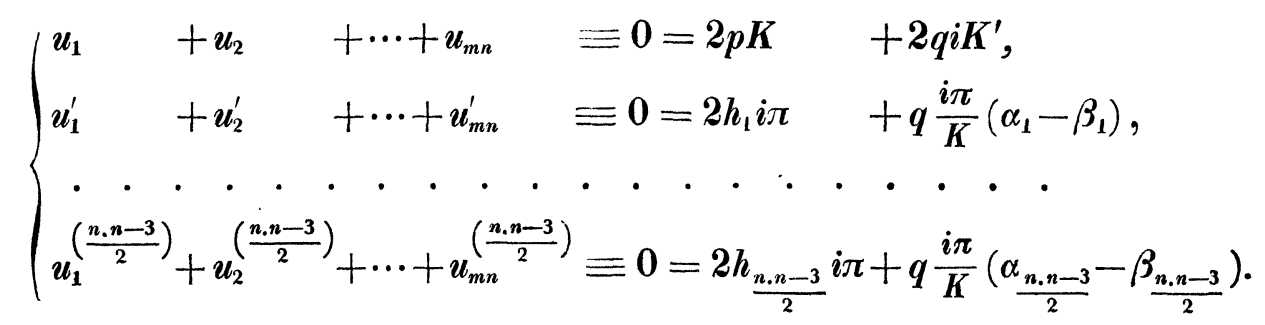

oder nach IV., wenn die Curve $m^{\text {ter }}$ Ordnung durch $\frac{n . n-3}{2}-\mu$ Doppelpunkte geht, und die Indices $1,2, \ldots \mu$ sich auf die übrigen Doppelpunkte beziehen:

$\left(53^{*}.\right)$

$$
\begin{aligned}
& \left\{\begin{array}{l}
u_{1}+u_{2}+\cdots+u_{\lambda}=2 p K+2 q i K^{\prime}+\sum_{1}^{\mu}\left(\alpha_{h}+\beta_{h}\right), \\
u_{1}^{\prime}+u_{2}^{\prime}+\cdots+u_{\lambda}^{\prime}=2 h_{1} i \pi+\frac{q i \pi}{K}\left(\alpha_{1}-\beta_{i}\right) \\
+\sum_{i=1}^{i=\mu} \log \frac{H\left(\alpha_{1}-\alpha_{i}\right) H\left(\alpha_{1}-\beta_{i}\right)}{H\left(\beta_{1}-\alpha_{i}\right) H\left(\beta_{1}-\beta_{i}\right)}-\frac{2 \mu}{n . n-3} \sum_{i=1}^{i} \log \frac{H\left(\alpha_{1}-\alpha_{i}\right) H\left(\alpha_{1}-\beta_{i}\right)}{H\left(\beta_{1}-\alpha_{i}\right) H\left(\beta_{1}-\beta_{i}\right)}
\end{array}\right. \\
& \begin{array}{r}
u_{1}^{(\mu)}+u_{2}^{(\mu)}+\cdots+u_{\lambda}^{(\mu)}=2 h_{\mu} i \pi+\frac{q i \pi}{K}\left(\alpha_{\mu}-\beta_{\mu}\right) \\
+\sum_{i=1}^{i=\mu} \log \frac{H\left(\alpha_{\mu}-\alpha_{i}\right) H\left(\alpha_{\mu}-\beta_{i}\right)}{H\left(\beta_{\mu}-\alpha_{i}\right) H\left(\beta_{\mu}-\beta_{i}\right)}-\frac{2 \mu}{n . n-3} \sum_{i=1}^{i=\frac{n . n-3}{2}} \log \\
\quad(\lambda=n(m-n+3)+2 \mu),
\end{array}
\end{aligned}
$$

wo bei den Summen immer dasjenige Glied auszuschliessen ist, bei welchem das Argument einer der $\boldsymbol{H}$-Functionen verschwinden würdè. 
Man kann von diesen Gleichungen ausgehend, alle diejenigen Probleme behandeln, welche ich in meinem Aufsatz über die Anwendung der Abelschen Functionen in der Geometrie, Band 63, pag. 189 dieses Journals für Curven ohne Doppelpunkte, und welche ich in diesem Bande, pag. 43 für Curven mit $\frac{n-1 . n-2}{2}$ Doppelpunkten gelöst habe. Ich werde mich, da die Methode ganz dieselbe bleibt, begnügen, für den vorliegenden Fall die folgenden Sätze anzuführen.

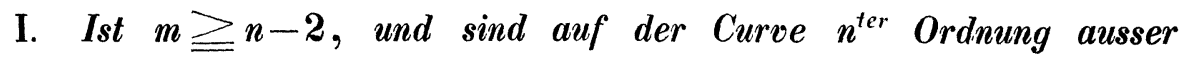
$\frac{n . n-3}{2}-\mu$ Doppelpunkten, noch

$$
m n-n(n-3)+2 u-r(\mu+1)
$$

Punkte gegeben, durch welche sämmtlich eine Curve $m^{\text {ter }}$ Ordnung gehen soll, während von den übrig bleibenden $\mu$ Doppelpunkten $\varkappa^{\prime}$ in Rückkehrpunkte übergehen, so kann man diese noch auf $r^{\mu+2-x^{\prime}}$ verschiedene Arten so legen, dass dieselbe mit der Curve $n^{\text {ter }}$ Ordnung in $" \imath+1$ verschiedenen Punkten eine $r$ punktige Berührung hat.

Legt man eine Curve $m^{\text {ter }}$ Ordnung durch die festen Punkte so wie durch jene $\frac{n . n-3}{2}-\mu$ Doppelpunkte, und lässt sie zugleich durch die Berührungspunkte von $r-1$ Curven gehen, welche aus obigem Problem entspringen, so schneidet sie die Curve $n^{\text {ter }}$ Ordnung noch immer in den Berïhrungspunkten einer $r^{\text {ten }}$ Curve derselben Art.

II. Nimmt man vr Punkte weniger als gegeben an, verlangt aber, dass die Curve $m^{\text {ter }}$ Ordnung in $\mu+\nu+1$ Punkten $r$-punktig berïhre, so

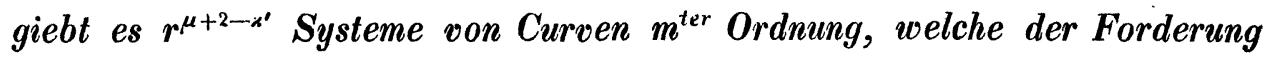
entsprechen.

Der vorige Satz über die Lage der Berïhrungspunkte besteht fort; insbesondere aber liegen die Berührungspunte von $r$ Curven desselben Systems mit den festen Punkten und den $\frac{n . n-3}{2}-\mu$ Doppelpunkten stets in einer Curve $m^{\text {ter }}$ Ordnung.

III. Ist insbesondere

$$
m n-n(n-3)+2 \mu-r(\mu+\nu+1)=0,
$$

so dass gar keine festen Punkte auf der Curve gegeben sind, so ist die Zahl der Systeme, wenn $m, r$ relative Primzahlen, gleich $r^{\mu+2-x^{\prime}} ;$ wenn aber

$$
m=m^{\prime} s, \quad r=r^{\prime} s,
$$


246 Clebsch, ebene Curven, deren Coord. elliptische Funct. eines Parameters sind.

wo $s>1$ und $m^{\prime}, r^{\prime}$ relative Primzahlen sind, so ist die Zahl der Systeme nur $\boldsymbol{r}^{\mu+2-x^{\prime}}-r^{\prime \mu+2-x^{\prime}}$.

Die Sätze über die Lage der Berührungspunkte bestehen fort.

Diese Sätze sind einfache Folgerungen aus den Gleichungen (53.), und man erhält sie aus den entsprechenden Sätzen der angeführten Abhandlung, wenn man die dort eintretende Periodenzahl $2 p$ durch die hier eintretende $\mu+2-\varkappa^{\prime}$ ersetzt.

Nur ein Umstand ist es, welcher den hier erhaltenen Lösungen einen besondern Charakter giebt. Die Perioden sind nämlich hier nicht gleichartig, indem die Perioden $2 K$ und $2 i K^{\prime}$ schon ihrer Entstehung nach sich wesentlich von den verschiedenen Perioden 2iл unterscheiden. Die Folge hievon ist, dass die Lösungen resp. Systeme, deren Anzahl im Allgemeinen immer $r^{\mu+2-x^{\prime}}$ war, sich von vorn herein in $r^{2}$ Gruppen theilen, deren Glieder durch gemeinschaftliche Werthe von $p, q$ verbunden sind. Auch vertheilt sich die Erniedrigung, welche die Zahl $r^{\mu+2-\alpha^{\prime}}$ bei III. erfährt, wenn $m, r$ nicht relative Primzahlen sind, auf diese Gruppen keineswegs gleichmässig, vielmehr gilt dabei, wie man sofort sieht, folgender Satz:

Die Systeme III. theilen sich in $r^{2}$ Gruppen, von denen die $r^{2}-r^{\prime 2}$ Gruppen, für welche $p, q$ nicht gleichzeitig durch $s$ theilbar sind, je $r^{\mu-x^{\prime}}$ Systeme enthalten, während in den $r^{\prime 2}$ übrigen Gruppen nur je $r^{\mu-\varkappa^{\prime}}-r^{\prime \mu-x^{\prime}}$ wirkliche Systeme vorkommen.

Die geometrische Bedeutung dieser Gruppirung kann man dadurch ausdrücken, dass, wenn man eine Curve $m^{\text {ter }}$ Ordnung durch die $\frac{n . n-3}{2}-\mu$ Doppelpunkte, durch die festen Punkte, und durch die Berïhrungspunkte von $r-1$ Curven derselben Gruppe legt, diese noch in den Beriihrungspunkten einer $\boldsymbol{r}^{\text {ten }}$ Curve derselben Gruppe schneidet.

\$. 16.

Berührungscurven $(n-3)^{\text {ter }}$ Ordnung.

Das Problem der Doppeltangenten der Curven vierter Ordnung, und die Erweiterung, welche ich demselben a. a. 0. für Curven $n^{\text {ter }}$ Ordnung gegeben habe, führt hier auf die im $\$ .13$ behandelte analytische Aufgabe. Man setzt $m=n-3$, und sucht diejenigen Curven $(n-3)^{\text {ter }}$ Ordnung, welche durch $\frac{n . n-3}{2}-\mu$ Doppelpunkte gehen, und ausserdem noch die Curve $n^{\text {ier }}$ Ordnung berühren, wo sie derselben begegnen, d. h. in $\mu$ Punkten. Man lässt also 
die $u$ paarweise zusammenfallen, und findet aus $\left(53^{*}\right.$.) nach Division mit 2:

$$
\begin{aligned}
& u_{1}+u_{2}+\cdots+u_{\mu} \equiv p K+q i K^{\prime}+\frac{1}{2} \sum_{1}^{\mu}\left(\alpha_{h}+\beta_{h}\right), \\
& u_{1}^{\prime}+u_{2}^{\prime}+\cdots+u_{\mu}^{\prime} \equiv h_{1} i \pi+\frac{q i \pi}{2 K}\left(\alpha_{1}-\beta_{1}\right)+\frac{1}{2} \sum_{k=1}^{k=1} \log \frac{H\left(\alpha_{1}-\alpha_{k}\right)}{H\left(\beta_{1}-\alpha_{k}\right)} \frac{\left.\alpha_{1}-\beta_{k}\right)}{H\left(\beta_{1}-\beta_{k}\right)}, \\
& \cdot \cdot \cdot \cdot \cdot \cdot \cdot \cdot \cdot \cdot \cdot \cdot \cdot \cdot \cdot \cdot \cdot \cdot \cdot \cdot \cdot \cdot \cdot \cdot \cdot \cdot \cdot \cdot \cdot \\
& u_{1}^{(\mu)}+u_{2}^{(\mu)}+\cdots+u_{\mu}^{(\mu)} \equiv h_{\mu} i \pi+\frac{q i \pi}{2 K}\left(\alpha_{\mu}-\beta_{\mu}\right)+\frac{1}{2} \sum_{k=1}^{k} \log \frac{H\left(\alpha_{\mu}-\alpha_{k}\right) H\left(\alpha_{\mu}-\beta_{k}\right)}{H\left(\beta_{\mu}-\alpha_{k}\right)} \cdot \boldsymbol{H ( \beta _ { \mu } - \beta _ { k } )}
\end{aligned}
$$

Dies sind in der That die Gleichungen welche in $\$ .13$ zu Grunde gelegt wurden. Nun zeigte sich dort, dass die Erfüllbarkeit dieser Gleichungen an die Bedingung geknüpft war:

$$
(q+1)(p+1)+h_{1}+h_{2}+\cdots+h_{\mu} \equiv \iota+1 \quad(\bmod 2) .
$$

Von den Grössen $q, p, h$, welche sämmtlich die Werthe 0 und 1 annehmen können, ist also eine durch die übrigen bestimmt, und die Anzahl der Lösungen wird also $2^{u+1}$. Sind aber unter den ausgeschlossenen Doppelpunkten noch $\varkappa^{\prime}$ Rückkehrpunkte vorhanden, so sind in den obigen Gleichungen ebensoviele Grössen $h$ auszulassen, und die Zahl der Lösungen beträgt nur $2^{\mu+1-x^{\prime}}$.

Hieraus, und aus Betrachtungen, wie sie am Schlusse von $\$ .8$ der angeführten Abhandlung angestellt sind, ergiebt sich folgender Salz:

IV. Es giebt für eine Curve $n^{\text {ter }}$ Ordnung mit $\frac{n \cdot n-3}{2}$ Doppel- resp. Riuckkehrpunkten $2^{\mu+1-\varkappa^{\prime}}$ Curven $(n-3)^{\text {ter }}$ Ordnung, welche durch $\frac{n . n-3}{2}-\mu$ dieser Doppelpunkte, von denen $x^{\prime}$ in Rückkehrpunkte übergehen, hindurchgehen, und zugleich die Curve noch in $\mu$ Punkten berühren.

Insbesondere, wenn $\mu=\frac{n . n-3}{2}$, und also die Curve $(n-3)^{\text {ter }}$ Ordnung durch keinen der Doppelpunkte geht:

Es giebt $2^{\frac{n . n-3}{2}+1-x}$ Curven $(n-3)^{\text {ter }}$ Ordnung, welche eine Curve $n^{\text {ter }}$ Ordnung mit $\frac{n . n-3}{2}-\varkappa$ Doppelpunkten und $*$ Rückkehrpunkten berühren, wo sie derselben begegnen, also in $\frac{n . n-3}{2}$ Punkten.

Unter den $2^{\frac{n-1 . n-2}{2}+1-x}$ Systemen von Curven $2 h-n-3^{\text {ter }}$ Ordnung $(h>n-3)$, welche bei geradem $n$ die gegebene Curve in $h n-\frac{n \cdot n-3}{2}$ Punkten berïhren, hat die Hälfte die Eigenschaft, dass ihre Berihrungspunkte mit den Beriihrungspunkten einer von den angefühten Curven $(n-3)^{\text {rer }}$ Ordnung in einer Curve 
248 Clebsch, ebene Curven, deren Coord. elliptische Funct. eines Parameters sind.

$h^{\text {ter }}$ Ordnung liegen. Die Zähl $2^{\frac{n-1 \cdot n-2}{2}+1-x}$ ist, wenn $n$ ungerade, um 1 zu vermindern; und dabei vermindert sich um 1 die Zahl derjenigen Systeme, welche die angegebene Eigenschaft nicht hat.

Ueber die gegenseitige Lage der Berührungspunkte dieser Curven $(n-3)^{\text {ter }}$ Ordnung finden noch weitere Sätze statt. Betrackten wir z. B. die Systeme von Curven $2(n-3)^{\text {ter }}$ Ordnung, welche die gegebene Curve in $n . n-3$ Punkten berühren. Für die Berührungspunkte finden dann nach (53.) folgende Gleichungen statt:

$$
\begin{aligned}
u_{1}+u_{2}+\cdots+u_{n, n-3} & \equiv P K+Q i K^{\prime}, \\
u_{1}^{(h)}+u_{2}^{(h)}+\cdots+u_{n . n-3}^{(h)} & \equiv H_{h} i \pi+Q \frac{i \pi\left(\alpha_{h}-\beta_{h}\right)}{K},
\end{aligned}
$$

wobei der Index $h$ sich auf diejenigen $\frac{n . n-3}{2}-\varkappa$ Doppelpunkte bezieht, welche nicht in Rückkehrpunkte übergehen. Die ganzen Zahlen $H, P, Q$ dürfen sämmtlich die Werthe 0 oder 1 annehmen, nur nicht gleichzeitig alle den Werth 0 , weil dann die obigen Gleichungen nicht mehr $n . n-3$ Berührungspunkte einer Curve $2(n-3)^{\text {ter }}$ Ordnung, sondern $n$. $n-3$ Schnittpunkte einer Curve $(n-3)^{\text {ter }}$ Ordnung angeben. Es giebt also $2^{\frac{n . n-3}{2}+2-x}-1$. solcher Systeme. Untersuchen wir nun, wie oft in einem solchen System eine Curve in zwei Berührungscurven $(n-3)^{\text {ter }}$ Ordnung zerfallen kann. Bezeichnen wir die den letztern entsprechenden ganzen Zahlen durch $p, q, h$ und $p^{\prime}, q^{\prime}, h^{\prime}$, so hat man offenbar die Bedingungen:

(54.) $\quad p+p^{\prime}=P, \quad q+q^{\prime}=Q, \quad h_{i}+h_{i}^{\prime}=H_{i}$,

aber ausserdem auch

$$
\begin{cases}(p+1)(q+1)+\Sigma h \equiv \frac{n-1 \cdot n-2}{2} & (\bmod .2) \\ \left(p^{\prime}+1\right)\left(q^{\prime}+1\right)+\Sigma h^{\prime} \equiv \frac{n-1 \cdot n-2}{2} & (\bmod .2)\end{cases}
$$

Trägt man nun die Werthe von $p^{\prime}, q^{\prime}, h^{\prime}$ in die Summe der beiden Gleichungen (55.) ein, so bleibt

(56.) $\quad q P+p Q \equiv P Q+P+Q+\Sigma H \quad(\bmod .2)$.

Man sieht hieraus, dass die $2^{\frac{n, n-3}{2}+2-x}$ Systeme sich in drei Classen theilen.

1. $P=0, Q=0$ und $\Sigma H \equiv 0$. Diese Classe umfasst, da man $\frac{n . n-3}{2}-x-1$ Grössen $H$ beliebig gleich 0 oder 1 setzt, aber nicht alle zu- 
gleich verschwinden lassen darf, $2^{\frac{n . n-3}{2}-x-1}-1$ Systeme. In jedem dieser Systeme darf man für $p, q, h$ noch die Zahlen einer beliebigen Curve $(n-3)^{\text {trer }}$ Ordnung wählen. Folglich ordnen sich für jedes der $2^{\frac{n . n-3}{2}-x-1}-1$ Systeme die $2^{\frac{n . n-3}{2}-x+1}$ Curven $(n-3)^{\text {ter }}$ Ordnung paarweise so, dass jedes der $2^{\frac{n . n-3}{2}-x}$ Paare eine Curve $2(n-3)^{\text {ler }}$ Ordnung des Systems wird, und dass also in diesen Systemen überhaupt $2^{\frac{n \cdot n-3}{2}-x}\left(2^{\frac{n . n-3}{2}-x-1}-1\right)$ Paare vorkommen.

2. $P=0, Q=0, \Sigma H \equiv 1$. Diese Classe umfasst $2^{\frac{n \cdot n-3}{2}-x-1}$ Systeme. Für sie wird die Gleichung (56.) unmöglich, und sie enthalten also keine Curvenpaare der $(n-3)^{\text {ten }}$ Ordnung.

3. Eine der Zahlen $P, Q$ von Null verschieden. Diese Classe umfasst $3.2^{\frac{n . n-3}{2}-x}$ Systeme, da alle $H$ willkürlich sind, und $P, Q$ noch die Werthe 0,$1 ; 1,0 ; 1,1$ annehmen können. In jedem dieser Systeme kann man eine der Zahlen $p, q$ beliebig wählen, ebenso sämmtliche $H$ bis auf eines, wodurch denn alles bestimmt ist. In jedem System kommen also $2^{\frac{n . n-3}{2}-x}$ Curven $(n-3)^{\text {ter }}$ Ordnung vor, welche für jedes System $2^{\frac{n \cdot n-3}{2}-x-1}$ Paare ergeben, deren jedes eine Curve $2(n-3)^{\text {ter }}$ Ordnung ersetzen kann.

In Bezug auf die Berührungspunkte der Curven $(n-3)^{\text {ten }}$ Ordnung ergiebt sich, da die Berührungspunkte zweier demselben System angehörigen Paare immer auf einer Curve.2(n-3) $)^{\text {ter }}$ Ordnung liegen, folgender Satz:

Die $2^{\frac{n . n-3}{2}-x+1}$ Curven $(n-3)^{t_{e} r}$ Ordnung, welche die gegebene Curve in $\frac{n . n-3}{2}$ Punkten beriuhren, bilden im Ganzen $2^{\frac{n . n-3}{2}-x}\left(2^{\frac{n n-3}{2}-x+1}-1\right)$ Paare. Diese Paare theilen sich zunächst in zwei grosse Classen, von denen die erste $2^{\frac{n . n-3}{2}-x}\left(2^{\frac{n . n-3}{2}-x-1}-1\right)$, die zweite $3.2^{\frac{n . n-3}{2}-x} \cdot 2^{\frac{n . n-3}{2}-x-1}$ Paare enthält. Die erste Classe zerfällt wieder in $2^{\frac{n \cdot n-3}{2}-x-1}-1$ Systeme von je $2^{\frac{n \cdot n-3}{2}-x}$ Paaren, dergestalt dass die Paare jedes Systems zusammen alle Curven $(n-3)^{\text {ter }}$ Ordnung, jede nur einmal gerechnet, enthalten; die Beriihrungspunkte je zweier Paare desselben Systems liegen auf einer Curve $2(n-3)^{\text {ter }}$ Ordnung. Die zweite Classe zerfällt in $3.2^{\frac{n . n-3}{2}-x}$ Systeme von je $2^{\frac{n . n-3}{2}-x-1}$ Paaren; die Paare eines Systems bilden zusammen die Hälfte aller Curven $(n-3)^{\text {ter }}$ OrdJourual für Mathematik Bd. LXIV. Heft. 3. 
250 Clebsch, ebene Curven, deren Coord. elliptische Funct. eines Parameters sind.

nung; und wieder liegen die Berïhrungspunkte zweier Paare desselben Systems auf einer Curve $2(n-3)^{\text {ter }}$ Ordnung.

Da hiebei jede Curve $2(n-3)^{\text {ter }}$ Ordnung offenbar dreimal auftritt, entsprechend den drei Zerlegungen von vier Curven $(n-3)^{\text {ter }}$ Ordnung in zwei Paare, so giebt es im Ganzen

$$
\frac{1}{3} \cdot 2^{\frac{n . n-3}{2}-x-1} \cdot\left(2^{\frac{n . n-3}{2}-x-1}-1\right) \cdot\left(5 \cdot 2^{\frac{n . n-3}{2}-x-1}-1\right)
$$

Curven $2(n-3)^{\text {ter }}$ Ordnung, welche sich durch die Berührungspunkte von je vier Curven $(n-3)^{\text {ter }}$ Ordnung legen lassen.

Die obigen Sätze werden illusorisch, sohald, was bei Curven vierter, fünfter und sechster Ordnung noch geschehen kann, alle Doppelpunkte in Rückkehrpunkte übergehen, oder doch nur ein einziger wirklicher Doppelpunkt übrig bleibt. Im zweiten Fall giebt es nur ein einziges Paar von Curven $(n-3)^{\text {ter }}$ Ordnung, welches zugleich das einzige System der zweiten Gruppe ist, während von der ersten Gruppe nichts übrig bleibt. Im ersten Fall dagegen, wo überhaupt kein $h$ mehr vorhanden ist, mnss man auf die Gleichung

$$
(p+1)(q+1) \equiv \frac{n-1 \cdot n-2}{2}(\bmod .2)
$$

zurückgehen, und findet sofort folgende specielle Resultate:

Es giebt eine Doppeltangente für eine Curve vierter Ordnung mit swei Rückkehrpunkten.

Es giebt drei Kegelschnitte, welche eine Curve funfter Ordnung mit füf Rückkehrpunkten in füf Punkten beriihren, und die funfzelon Berïhrungspunkte liegen auf einer Curve dritter Ordnung.

Es giebt drei Curven dritter Ordnung, welche eine Curve sechster Ordnung mit neun Ruickkehrpunkten in neun Punkten beriihren.

\$. 17.

Curven vierter Ordnung mit zwei Doppelpunkten.

Als Beispiel für diese Untersuchungen werde ich die Curven vierter Ordnung mit zwei Doppelpunkten behandeln. Die Axe $x_{1}$ sei die Verbindungslinie der Doppelpunkte, die Axe der $x_{2}$ gehe durch den einen Doppelpunkt, die Axe der $x_{3}$ durch den andern; die Gleichung eines Paars von Geraden, welche durch die andern Schnittpunkte der Axen $x_{2}, x_{3}$ mit der Curve geht, sei $A . C=0$; dann nimmt die Gleichung der Curve die Form̄ an

(57.) $f=x_{2}^{2} x_{3}^{2}+2 x_{1} x_{2} x_{3} B+x_{1}^{2} A C=0$, 
wo $A, B, C$ die linearen Ausdrücke bedeuten:

$$
\begin{aligned}
& A=a_{1} x_{1}+a_{2} x_{2}+a_{3} x_{3}, \\
& B=b_{1} x_{1}+b_{2} x_{2}+b_{3} x_{3}, \\
& C=c_{1} x_{1}+c_{2} x_{2}+c_{3} x_{3} .
\end{aligned}
$$

Der Kegelschnittbüschel, durch den man die Punkte der Curve parweise bestimmt, sei

$$
\text { (58.) } \quad x_{2} x_{3}+\lambda x_{1} A=0 \text {; }
$$

er geht durch die Doppelpunkte, und durch die festen Punkte $x_{2}=0, A=0$ und $x_{3}=0, A=0$, welche auf der Curve liegen. Verbinden wir die Gleichungen (57.), (58.) mit einander, so erhalten wir an Stelle von $f=0$ die Gleichung:

$$
\text { (59.) } \lambda^{2} A-2 \lambda B+C=0 \text {, }
$$

welche für die $x$ vom ersten Grade ist. Sie stellt die Verbindungslinie des beweglichen Punktenpaars dar, in welchem ein Kegelschnitt des Systems (58.) die Curve $f=0$ schneidet, und die Curve, welche von dieser Geraden umhüllt wird, ist also der Kegelschnitt

$$
A C-B^{2}=0 .
$$

Derselbe hat die Gerade zur Tangente, welche durch die gewählten Grundpunkte geht $(A=0)$; aber auch $C=0$, welches die Punkte verbindet, in denen zwei von den Doppelpunkten nach den festen Punkten gezogenen Gerade die Curve treffen.

Legt man jetzt einen Büschel $P+\varrho Q=0$, dessen Strahlen nach den beweglichen Schnittpunkten gerichtet sind, so hat man $\varrho$ als Function von $\lambda$, und die $x$ als Functionen von $\lambda$ und $\rho$ darzustellen. Aus (59.) und aus der Gleichung des Buschels ergiebt sich:

$$
\left\{\begin{array}{l}
\sigma x_{1}=\left(p_{2}+\varrho q_{2}\right)\left(\lambda^{2} a_{3}-2 \lambda b_{3}+c_{3}\right)-\left(p_{3}+\varrho q_{3}\right)\left(\lambda^{2} a_{2}-2 \lambda b_{2}+c_{2}\right), \\
\sigma x_{2}=\left(p_{3}+\varrho q_{3}\right)\left(\lambda^{2} a_{1}-2 \lambda b_{1}+c_{1}\right)-\left(p_{1}+\varrho q_{1}\right)\left(\lambda^{2} a_{3}-2 \lambda b_{3}+c_{3}\right), \\
\sigma x_{3}=\left(p_{1}+\varrho q_{1}\right)\left(\lambda^{2} a_{2}-2 \lambda b_{2}+c_{2}\right)-\left(p_{2}+\varrho q_{2}\right)\left(\lambda^{2} a_{1}-2 \lambda b_{1}+c_{1}\right),
\end{array}\right.
$$

und wenn man diese Ausdrücke in (58.) einführt, findet sich die quadratische Gleichung durch welche $\varrho$ als Function von $\lambda$ gegeben ist. - Diese Gleichung hat die Form

wo

$$
\text { (61.) } P+2 R \rho+Q \rho^{2}=0 \text {, }
$$

$$
\text { (62.) } \quad\left\{\begin{array}{l}
\boldsymbol{P}=\Sigma \Sigma \omega_{i k} p_{i} p_{k}, \\
\boldsymbol{R}=\Sigma \Sigma \omega_{i k} p_{i} q_{k}, \\
\boldsymbol{Q}=\Sigma \Sigma \omega_{i k} q_{i} \boldsymbol{q}_{k} .
\end{array}\right.
$$


Und wenn man der Kürze wegen setzt:

$$
\left\{\begin{array}{l}
L_{1}=\lambda^{2} a_{1}-2 \lambda b_{1}+c_{1} \\
L_{2}=\lambda^{2} a_{2}-2 \lambda b_{2}+c_{2} \\
L_{3}=\lambda^{2} a_{3}-2 \lambda b_{3}+c_{3}
\end{array}\right.
$$

so ist

$$
\begin{cases}\omega_{11}=-2 L_{2} L_{3}, & \omega_{23}=-L_{1}^{2}-\lambda L_{2}\left(a_{1} L_{3}-a_{3} L_{1}\right)-\lambda L_{3}\left(a_{1} L_{2}-a_{2} L_{1}\right), \\ \omega_{22}=2 \lambda L_{3}\left(a_{1} L_{3}-a_{3} L_{1}\right), & \omega_{31}=L_{1} L_{2}-\lambda L_{2}\left(a_{3} L_{2}-a_{2} L_{3}\right) \\ \omega_{23}=2 \lambda L_{2}\left(a_{1} L_{2}-a_{2} L_{1}\right), & \omega_{12}=L_{1} L_{3}+\lambda L_{3}\left(a_{3} L_{2}-a_{2} L_{3}\right) .\end{cases}
$$

Wegen der Gleichungen, welche hieraus folgen:

$$
\begin{aligned}
& \omega_{11} L_{1}+\omega_{12} L_{2}+\omega_{13} L_{3}=0 \\
& \omega_{21} L_{1}+\omega_{22} L_{2}+\omega_{23} L_{3}=0 \\
& \omega_{31} L_{1}+\omega_{32} L_{2}+\omega_{33} L_{3}=0
\end{aligned}
$$

kann man die Unterdeterminanten $W_{i k}$ in die Form bringen

und man findet

$$
W_{i k}=-M \cdot L_{i} L_{k},
$$

(65.) $\quad M=L_{1}^{2}+\lambda^{2}\left(a_{3} L_{2}-a_{2} L_{3}\right)^{2}+4 \lambda a_{1} L_{2} L_{3}-2 \lambda L_{1}\left(a_{3} L_{2}+a_{2} L_{3}\right)$.

Die Determinante der Gleichung (61.) wird also

und man hat

$$
Q^{2}-P R=M \cdot\left(\Sigma \pm L_{1} p_{2} q_{3}\right)^{2}
$$

$$
\text { (66.) } \quad \rho=\frac{1}{Q}\left\{-R+\left(\Sigma \pm L_{1} p_{2} q_{3}\right) \sqrt{ } M\right\},
$$

, wobei unter der Wurzel nur noch ein Ausdruck vierten Grades in $\lambda$ steht, wie es sein muss.

Die Einführung dieser Ausdrücke giebt, nach einer Reduction wie in \$. 4, die folgenden Werthe der $x$ :

$$
\text { (67.) } \quad\left\{\begin{array}{l}
\sigma x_{1}=\omega_{11} q_{1}+\omega_{12} q_{2}+\omega_{13} q_{3}-\left(q_{2} L_{3}-q_{3} L_{2}\right) \sqrt{M}, \\
\sigma x_{2}=\omega_{12} q_{1}+\omega_{22} q_{2}+\omega_{23} q_{3}-\left(q_{3} L_{1}-q_{1} L_{3}\right) \sqrt{M}, \\
\sigma x_{3}=\omega_{13} q_{1}+\omega_{23} q_{2}+\omega_{33} q_{3}-\left(q_{1} L_{2}-q_{2} L_{1}\right) \sqrt{M} .
\end{array}\right.
$$

Da indessen die Coordinate $x_{1}$ ohnedies vor den andern bevorzugt ist, so kann man ohne weitere Verletzung der Symmetrie $q_{2}=q_{3}=0$ setzen, was damit übereinkommt, dass das Centrum des Büschels in die Verbindungslinie der Doppelpunkte gelegt wird. Man erhält dann : 


$$
\left\{\begin{array}{l}
\sigma x_{1}=-2 L_{2} L_{3}, \\
\sigma x_{2}=L_{3}\left\{L_{1}+\lambda\left(a_{3} L_{2}-a_{2} L_{3}\right)+\sqrt{M}\right\} \\
\sigma x_{3}=L_{2}\left\{L_{1}-\lambda\left(a_{3} L_{2}-a_{2} L_{3}\right)-\sqrt{M}\right\} .
\end{array}\right.
$$

Die rechten Theile dieser Gleichungen verschwinden nun sämmtlich, wie schon oben gezeigt ist, für gewisse Werthe von $\lambda$, welche der Frage fremd sind. Bemerkt man nämlich die identischen Gleichungen:

$$
\begin{aligned}
& \left\{L_{1}+\lambda\left(a_{3} L_{2}-a_{2} L_{3}\right)\right\}^{2}-M=4 \lambda L_{2}\left(a_{3} L_{1}-a_{1} L_{3}\right), \\
& \left\{L_{1}-\lambda\left(a_{3} L_{2}-a_{2} L_{3}\right)\right\}^{2}-M=4 \lambda L_{3}\left(a_{2} L_{1}-a_{1} L_{2}\right),
\end{aligned}
$$

so sieht man, dass alle rechten Theile der Gleichungen (68.) verschwinden, wenn

$$
\begin{aligned}
& L_{2}=0 \quad \text { und } \quad \sqrt{M}=-\left\{L_{1}+\lambda\left(a_{3} L_{2}-a_{2} L_{3}\right)\right\}, \\
& L_{3}=0 \quad \text { und } \quad \sqrt{M}=\quad\left\{L_{1}-\lambda\left(a_{3} L_{2}-a_{2} L_{3}\right)\right\} \text {. }
\end{aligned}
$$

Man hat also im Ganzen vier Werthe von $L$, und für jeden derselben hat die Quadratwurzel ein bestimmtes Vorzeichen.

Die Werthe, für welche die $x$ einzeln verschwinden, erhält man aus den Gleichungen:

$$
\begin{array}{ccl}
\text { für } x_{1}: & L_{2}=0 & \sqrt{ } M=\left\{L_{1}+\lambda\left(a_{3} L_{2}-a_{2} L_{3}\right)\right\} \\
& L_{3}=0 & \sqrt{ } M=-\left\{L_{1}-\lambda\left(a_{3} L_{2}-a_{2} L_{3}\right)\right\} ; \\
\text { für } x_{2}: & L_{3}=0 & \sqrt{ } M=-\left\{L_{1}-\lambda\left(a_{3} L_{2}-a_{2} L_{3}\right)\right\} \\
& \lambda=0 & \sqrt{ } M=-L_{1} \\
& a_{3} L_{1}-a_{1} L_{3}=0 & \sqrt{ } M=-\left\{L_{1}+\lambda\left(a_{3} L_{2}-a_{2} L_{3}\right)\right\} ; \\
\text { für } x_{3}: & L_{2}=0 & \sqrt{ } M=-\left\{L_{1}+\lambda\left(a_{3} L_{2}-a_{2} L_{3}\right)\right\} \\
& \lambda=0 & \sqrt{ } M=L_{1} \\
a_{2} L_{1}-a_{1} L_{2}=0 & \sqrt{ } M=\left\{L_{1}-\lambda\left(a_{3} L_{2}-a_{2} L_{3}\right)\right\} .
\end{array}
$$

\section{ऽ. 18.}

Einführung der elliptischen Functionen in die Coordinatenausdrücke der Curven vierter Ordnung mit zwei Doppelpunkten.

Bezeichnen wir nun die Wurzeln von $M=0$ durch $m_{1}, m_{2}, m_{3}, m_{4}$, und setzen

$$
\begin{aligned}
\sin ^{2} \mathrm{am} u & =\frac{m_{3}-m_{1}}{m_{3}-m_{2}} \cdot \frac{\lambda-m_{2}}{\lambda-m_{1}}, \\
\cos ^{2} \mathrm{am} u & =\frac{m_{1}-m_{2}}{m_{3}-m_{2}} \cdot \frac{\lambda-m_{3}}{\lambda-m_{1}}, \quad k^{2}=\frac{m_{3}-m_{2} \cdot m_{4}-m_{1}}{m_{3}-m_{1} \cdot m_{4}-m_{2}}, \\
\Delta \mathrm{am} u & =\frac{m_{1}-m_{2}}{m_{4}-m_{2}} \cdot \frac{\lambda-m_{4}}{\lambda-m_{1}},
\end{aligned}
$$


254 Clebsch, bene Curben, déren Coord. elliptische Funct. eines Parameters sind.

so gehört jedem der obigen Verschwindungswerthe ein bestimmter Werth von $u$ an. Diejenigen Werthe von $u$, für welche nur je eines der $x$ verschwindet, seien:

für $x_{1}: \xi_{2}, \eta_{2}\left(\right.$ aus $L_{2}=0$ ), $\xi_{3}, \eta_{3}\left(\right.$ aus $L_{3}=0$ ),

für $x_{2}: \quad \xi_{3}, \eta_{3}\left(\right.$ aus $\left.L_{3}=0\right), \quad \zeta$ (aus $\lambda=0$ ), $\quad \zeta_{2}\left(\right.$ aus $a_{3} L_{1}-a_{1} L_{3}=0$ ),

für $x_{3}: \xi_{2}, \eta_{2}\left(\right.$ aus $\left.L_{2}=0\right),-\zeta$ (aus $\lambda=0$ ), $\zeta_{3}$ (aus $a_{2} L_{1}-a_{1} L_{2}=0$ ).

Diejenigen Werthe von $u$, für welche die rechten Theile sämmtlicher Gleichungen (68.) verschwinden, sind dann:

$$
-\xi_{2}, \quad-\eta_{2}, \quad-\xi_{3}, \quad-\eta_{3} \text {. }
$$

Abstrahirt man also von den gemeinschaftlichen Factoren, so kann man setzen:

$$
\begin{aligned}
& \sigma x_{1}=c_{1} \cdot \boldsymbol{H}\left(u-\xi_{2}\right) \boldsymbol{H}\left(u-\eta_{2}\right) \boldsymbol{H}\left(u-\xi_{3}\right) \boldsymbol{H}\left(u-\eta_{3}\right), \\
& \sigma \boldsymbol{x}_{2}=\boldsymbol{c}_{2} \cdot \boldsymbol{H}\left(u-\xi_{3}\right) \boldsymbol{H}\left(u-\eta_{3}\right) \boldsymbol{H}(u-\zeta) \boldsymbol{H}\left(u-\zeta_{2}\right), \\
& \sigma \boldsymbol{x}_{3}=c_{3} \cdot \boldsymbol{H}\left(u-\xi_{2}\right) \boldsymbol{H}\left(u-\eta_{3}\right) \boldsymbol{H}(u+\zeta) \boldsymbol{H}\left(u-\zeta_{3}\right) .
\end{aligned}
$$

Da ferner die Summe aller Verschwindungswerthe desselben algebraischen Ausdrucks immer Null ist (oder doch einer Periode congruent), so finden noch die Gleichungen statt:

$$
\xi_{2}+\eta_{2}+\xi_{3}+\eta_{3} \equiv \xi_{3}+\eta_{3}+\zeta+\zeta_{2} \equiv \xi_{2}+\eta_{2}-\zeta+\zeta_{3} \equiv 4 \varepsilon
$$

wenn $4 \varepsilon$ den gemeinsamen Werth dieser Summen bezeichnet. Aus diesen Gleichungen folgt noch:

$$
\zeta_{2}+\zeta_{3} \equiv 4 \varepsilon, \quad \xi_{2}+\eta_{2} \equiv \zeta+\zeta_{2}, \quad \xi_{3}+\eta_{3} \equiv-\zeta+\zeta_{3} .
$$

Man kann also drei neue Argumente $\sigma, \delta_{2}, \delta_{3}$ einführen, so dass durch sie und $\zeta$ selbst die andern Argumente sich folgendermassen ausdrücken:

$$
\begin{array}{ll}
\xi_{2}=\varepsilon+\frac{\zeta+\sigma}{2}+\delta_{2}, & \xi_{3}=\varepsilon-\frac{\zeta+\sigma}{2}+\delta_{3}, \\
\eta_{2}=\varepsilon+\frac{\zeta+\sigma}{2}-\delta_{2}, & \eta_{3}=\varepsilon-\frac{\zeta+\sigma}{2}-\delta_{3}, \\
\zeta_{2}=2 \varepsilon+\sigma, & \zeta_{3}=2 \varepsilon-\sigma .
\end{array}
$$

Wenn man jetzt $v=u-\varepsilon$ setzt, so erhält man:

$$
\begin{aligned}
& \sigma x_{1}=c_{1} H\left(v-\frac{\zeta+\sigma}{2}-\delta_{2}\right) H\left(v-\frac{\zeta+\sigma}{2}+\delta_{2}\right) H\left(v+\frac{\zeta+\sigma}{2}-\delta_{3}\right) H\left(v+\frac{\zeta+\sigma}{2}+\delta_{3}\right), \\
& \sigma x_{2}=c_{2} H\left(v+\frac{\zeta+\sigma}{2}-\delta_{3}\right) H\left(v+\frac{\zeta+\sigma}{2}+\delta_{3}\right) H(v-\zeta+\varepsilon) H(v-\varepsilon-\sigma), \\
& \sigma x_{3}=c_{3} H\left(v-\frac{\zeta+\sigma}{2}-\delta_{2}\right) H\left(v-\frac{\zeta+\sigma}{2}+\delta_{2}\right) H(v+\zeta+\varepsilon) H(v-\varepsilon+\sigma) .
\end{aligned}
$$


Die linken Theile verwandelt man leicht wieder in algebraische Ausdrücke; zunächst erhält man, indem man nur die Constanten und den Werth vơn $\sigma$ ändert:

$$
\text { (69.) }\left\{\begin{array}{l}
\sigma x_{1}=C_{1} \cdot\left[\sin ^{2} \mathrm{am}\left(v-\frac{\zeta+\sigma}{2}\right)-\sin ^{2} \mathrm{am}_{2}\right]\left[\sin _{2} \cdot\left[\sin ^{2} \mathrm{am}\left(v+\frac{\zeta+\sigma}{2}\right)-\sin ^{2} \mathrm{am} \delta_{3}\right]\left[\sin ^{2} \mathrm{am}\left(v-\frac{\zeta+\sigma}{2}\right)-\sin ^{2} \mathrm{am} \delta_{3}\right],\right. \\
\sigma x_{3}=C_{3} \cdot\left[\sin ^{2} \mathrm{am}\left(v-\frac{\zeta+\sigma}{2}\right)-\sin ^{2} \mathrm{am} \delta_{3}\right]\left[\sin ^{2} \mathrm{am}\left(v+\frac{\zeta+\sigma}{2}\right)-\sin ^{2} \mathrm{am}\left(\varepsilon+\frac{\zeta-\sigma}{2}\right)\right] .
\end{array}\right.
$$

Setzt man nun

$$
\begin{aligned}
& \sin ^{2} \operatorname{am} v=z, \quad \sin ^{2} \mathrm{am} \frac{\zeta+\sigma}{2}=p, \quad \sin ^{2} \mathrm{am} \delta_{2}=p_{2}, \quad \sin ^{2} \mathrm{am} \delta_{3}=p_{3}, \\
& \sin ^{2} \operatorname{am}\left(\varepsilon-\frac{\zeta-\sigma}{2}\right)=q_{2}, \quad \sin ^{2} \operatorname{am}\left(\varepsilon+\frac{\zeta-\sigma}{2}\right)=q_{3},
\end{aligned}
$$

so kommen endlich, bei nochmaliger zweckmässig gewählter Veränderung des Werthes von $\sigma$ folgende algebraische Formeln:

(70.)

$$
\left\{\begin{aligned}
\sigma x_{1} & =C_{1}\left\{(z-p)^{2}-\left(p_{2}+p_{3}\right)\left[z(1-p)\left(1-k^{2} p\right)+p(1-z)\left(1-k^{2} z\right)\right]\right. \\
& \left.+p_{2} p_{3}\left(1-k^{2} p z\right)^{2}-2\left(p_{2}-p_{3}\right) \sqrt{p .1-p .1-k^{2} p} \sqrt{z .1-z .1-k^{2} z}\right\} \\
\sigma x_{2} & =C_{2}\left\{(z-p)^{2}-\left(q_{2}+p_{3}\right)\left[z(1-p)\left(1-k^{2} p\right)+p(1-z)\left(1-k^{2} z\right)\right]\right. \\
& \left.+q_{2} p_{3}\left(1-k^{2} p z\right)^{2}-2\left(q_{2}-p_{3}\right) \sqrt{p .1-p .1-k^{2} p} \sqrt{z .1-z .1-k^{2} z}\right\} \\
\sigma x_{3}= & C_{3}\left\{(z-p)^{2}-\left(p_{2}+q_{3}\right)\left[z(1-p)\left(1-k^{2} p\right)+p(1-z)\left(1-k^{2} z\right)\right]\right. \\
& \left.+p_{2} q_{3}\left(1-k^{2} p z\right)^{2}-2\left(p_{2}-q_{3}\right) \sqrt{p .1-p .1-k^{2} p} \sqrt{z .1-z .1-k^{2} z}\right\}
\end{aligned}\right.
$$

Diese Formeln haben nun in der That den verlangten Charakter; ihre rechten Theile zerfalien je in eine rationale ganze Function zweiter Ordnung von $z$, und in ein Glied, welches aus $\sqrt{\overline{z .1-z .1-k^{2} z}}$ besteht, multiplicirt mit einer Constanten.

Der geometrischen Beziehung der Axen zu den gegebenen Curven wegen erkennt man sofort, dass folgende Werthe von $v$ den angegebenen Punkten entsprechen:

$$
\begin{aligned}
& \left.\left.\begin{array}{l}
\frac{\zeta+\sigma}{2}+\delta_{2} \\
\frac{\zeta+\sigma}{2}-\delta_{2}
\end{array}\right\} \begin{array}{l}
\text { dem im Durchschnitte von } x_{1}=0, x_{3}=0 \text { liegenden } \\
\text { Doppelpunkt, } \\
-\frac{\zeta+\sigma}{2}+\delta_{3} \\
-\frac{\zeta+\sigma}{2}-\delta_{3}
\end{array}\right\} \begin{array}{l}
\text { dem im Durchschnitte von } x_{1}=0, x_{2}=0 \text { liegenden } \\
\text { Doppelpunkt, }
\end{array}
\end{aligned}
$$




$$
\begin{array}{r}
\varepsilon+\sigma \text { dem Schnittpunkte von } x_{2}=0 \text { mit } A=0 \\
\varepsilon-\sigma \text { dem Schnittpunkte von } x_{3}=0 \text { mit } A=0, \\
\zeta-\varepsilon \text { dem Schnittpunkte von } x_{2}=0 \text { mit } C=0, \\
-\zeta-\varepsilon \text { dem Schnittpunkte von } x_{3}=0 \text { mit } C=0
\end{array}
$$

In der That verschwinden, wie man aus (68.) sieht, $A$ und $C$, ersteres wenn $a_{3} L_{1}-a_{1} L_{3}=0$ oder $a_{2} L_{1}-a_{1} L_{2}=0$, woraus die Argumente $\varepsilon+\sigma, \varepsilon-\sigma$ entsprangen, letzteres wenn $\lambda=0$, woraus die Argumente $\zeta-\varepsilon,-\zeta-\varepsilon$ entstanden sind. Man kann dies benutzen um die Verhältnisse der Constanten $C_{1}, C_{2}$, $C_{3} \mathrm{zu}$ bestimmen. Setzt man nämlich in (69.) $v=\varepsilon+\sigma$, so wird $x_{2}=0$ und

daher

$$
a_{1} x_{1}+a_{3} x_{3}=0
$$

$$
-\frac{a_{1}}{a_{3}}=\frac{C_{3}}{C_{1}} \cdot \frac{\sin ^{2} \mathrm{am}\left(\varepsilon+\frac{\zeta+3 \sigma}{2}\right)-\sin ^{2} \mathrm{am}\left(\varepsilon+\frac{\zeta-\sigma}{2}\right)}{\sin ^{2} \mathrm{am}\left(\varepsilon+\frac{\zeta+3 \sigma}{2}\right)-\sin ^{2} \mathrm{am} \delta_{3}}
$$

setzt man dagegen $v=\varepsilon-\sigma$, so wird $x_{3}=0, a_{1} x_{1}+a_{2} x_{2}=0$, also

$$
\text { (72.) }-\frac{a_{1}}{a_{2}}=\frac{C_{2}}{C_{1}} \cdot \frac{\sin ^{2} \operatorname{am}\left(\varepsilon-\frac{\zeta+3 \sigma}{2}\right)-\sin ^{2} \operatorname{am}\left(\varepsilon-\frac{\zeta-\sigma}{2}\right)}{\sin ^{2} \operatorname{am}\left(\varepsilon-\frac{\zeta+3 \sigma}{2}\right)-\sin ^{2} \operatorname{am} \delta_{2}} \text {. }
$$

Durch diese Formeln und (69.) ist die vollständige Darstellung der Coordinaten als Functionen von $v$ geleistet.

\section{\$. 19.}

Bedingungen für ein Schnittpunktsystem.

Ich komme jetzt zu den Formeln für ein Schnittpunktsystem, d. h. zu den Bedingungen, welche zwischen den Argumenten von $4 m$ Punkten stattfinden müssen, damit diese auf einer Curve $m^{\text {ter }}$ Ordnung liegen. Wir haben in den Formeln III. (\$. 9) nur für $\alpha_{1}, \alpha_{2}, \beta_{1}, \beta_{2}$ zu setzen:

$$
\frac{\zeta+\sigma}{2}+\delta_{2}, \quad \frac{\zeta+\sigma}{2}-\delta_{2},-\frac{\zeta+\sigma}{2}+\delta_{3},-\frac{\zeta+\sigma}{2}-\delta_{3} .
$$

Aus jenen Formeln findet man dann für den Fall, dass kein Punkt des Systems mit einem Doppelpunkte zusammenfällt: 


$$
\left\{\begin{array}{l}
v_{i=4 m}+v_{2}+\cdots+v_{4 m} \equiv 0, \\
\underset{i=1}{\prod_{1}} \frac{H\left(\frac{\zeta+\sigma}{2}+\delta_{2}-v_{i}\right)}{H\left(\frac{\zeta+\sigma}{2}-\delta_{2}-v_{i}\right)} \equiv\left\{\frac{H\left(\zeta+\sigma+\delta_{2}+\delta_{3}\right)}{H\left(\zeta+\sigma-\delta_{2}-\delta_{3}\right)} \cdot \frac{H\left(\zeta+\sigma+\delta_{2}-\delta_{3}\right)}{H\left(\zeta+\sigma-\delta_{2}+\delta_{3}\right)}\right\}^{m}, \\
\prod_{i=4 m}^{M} \frac{H\left(\frac{\zeta+\sigma}{2}-\delta_{3}+v_{i}\right)}{H\left(\frac{\zeta+\sigma}{2}+\delta_{3}+v_{i}\right)} \equiv\left\{\frac{H\left(\zeta+\sigma-\delta_{2}-\delta_{3}\right)}{H\left(\zeta+\sigma+\delta_{2}+\delta_{3}\right)} \cdot \frac{H\left(\zeta+\sigma+\delta_{2}-\delta_{3}\right)}{H\left(\zeta+\sigma-\delta_{2}+\delta_{3}\right)}\right\}^{m}
\end{array}\right.
$$

Dagegen, wenn ein Punkt des Systems mit dem Doppelpunkte $x_{2}=0, x_{1}=0$ zusammenfällt, also zwei der Argumente $v$ in $-\frac{\zeta+\sigma}{2}+\delta_{3}$ und $-\frac{\zeta+\sigma}{2}-\delta_{3}$ übergehen :

$$
\left\{\begin{array}{c}
v_{1}+v_{2}+\cdots+v_{4 m-2} \equiv \zeta+\sigma, \\
\prod_{i=1}^{i=4 m-2} \frac{H\left(\frac{\zeta+\sigma}{2}+\delta_{2}-v_{i}\right)}{H\left(\frac{\zeta+\sigma}{2}-\delta_{2}-v_{i}\right)} \equiv\left\{\frac{H\left(\zeta+\sigma+\delta_{2}+\delta_{3}\right) H\left(\zeta+\sigma+\delta_{2}-\delta_{3}\right)}{H\left(\zeta+\sigma-\delta_{2}-\delta_{3}\right) H\left(\zeta+\sigma-\delta_{2}+\delta_{3}\right)}\right\}^{m-1} .
\end{array}\right.
$$

Endlich, wenn die Curve $m^{\text {ter }}$ Ordnung durch beide Doppelpunkte gehen soll, bleibt nur die eine Gleichung übrig:

$$
v_{1}+v_{2}+\cdots+v_{4 m-4} \equiv 0 \text {. }
$$

§. 20.

Vierpunktig berührende Kegelschnitte, welche durch die Doppelpunkte gehen.

Die letzte Gleichung gestattet unmittelbar die Lösung einiger bemerkenswerthen Aufgaben. Es soll z. B. ein Kegelschnitt gefunden werden, welcher durch die Doppelpunkte geht, und die Curve noch vierpunktig beriihrt. Ist $v$ das Argument des Berührungspunktes, so findet man aus (75.)

$$
4 v \equiv 0, \quad v \equiv \frac{p K+q i K^{\prime}}{2} \text {. }
$$

Die Zahlen $p, q$ können hier die Werthe 0, 1, 2, 3 erhalten; es giebt also 16 Kegelschnitte dieser Art.

Bezeichnen wir, um die gegenseitige Lage der 16 Berührungspunkte genauer darzustellen, diese Kegelschnitte allgemein durch $p q$, so dass man die 16 Kegelschnitte hat

(76.) $\left\{\begin{array}{llll}\text { I. } & \text { II. } & \text { III. } & \text { IV. } \\ 00,02,20,22 ; & 01,03,21,23 ; & 10,12,30,32 ; & 11,13,31,33 .\end{array}\right.$ 
Die Kegelschnitte ordnen sich hier sofort in vier Gruppen, und zwar haben diese Gruppen die Eigenschaft, dass es immer einen Kegelschnitt giebt, der durch die Doppelpunkte geht, und die Curve vierter Ordnung in den Berührungspunkten von irgend zwei Kegelschnitten derselben Gruppe berihht; solcher neuen Kegelschnitte giebt es also $4.6=24$.

Die Berührungspunkte aller vier Berührungskegelschnitte derselben Gruppe liegen mit den Doppelpunkten in einem Kegelschnitt, so dass es vier solcher Kegelschnitte giebt.

Man kann ferner Kegelschnitte so legen, dass jeder derselben durch die Doppelpunkte geht, in dem Beriihrungspunkt eines der sechszehn Kegelschnitte berïht, und durch die Berïhrungspunkte zweier andern hindurchgeht. Die letzten beiden gehören dann immer einer andern Gruppe an, als der erste; die letzten beiden ändern sich nicht, wenn für den ersten alle Kegelschnitte einer Gruppe gesetzt werden, und jeder solchen Gruppe gegenüber sondern sich die andern drei Gruppen in Paare, so dass jedes der sechs Paare mit einem beliebigen Kegelschnitt der ersten Gruppe combinirt werden kann. Solcher Kegelschnitte giebt es also 4.4.6=96.

Die zu den in (76.) aufgeführten Gruppen gehörigen Paare sind folgende:

zu I.

zu II. zu III. zu IV.

(77.)

$\left\{\begin{array}{llll}01,03 ; 21,23 & 00,02 ; 20,22 & 00,20 ; 02,22 & 00,22 ; 02,20 \\ 10,30 ; 12,32 & 10,32 ; 12,30 & 01,23 ; 21,03 & 01,21 ; 03,23 \\ 11,33 ; 13,31 & 11,31 ; 13,33 & 11,13 ; 31,33 & 10,12 ; 30,32 .\end{array}\right.$

Durch die Berührungspunkte jedes dieser Paare und durch die Doppelpunkte gehen vier Kegelschnitte, welche beziehungsweise in den vier Berührungspunkten der entsprechenden Gruppe berühren.

Die in (77.) derselben Gruppe entsprechenden Paare haben die Eigenschaft, dass je zwei Paare mit den Doppelpunkten in einem Kegelschnitt liegen. Es giebt also $6.8=48$ Kegelschnitte, deren jeder die Doppelpunkte und zwei Paare von Berührungspunkten enthält, deren jedes einer anderen Gruppe angehört.

Endlich liegen 4.16=64 mal vier Berührungspunte, welche lauter verschiedenen Gruppen angehören, mit den Doppelpunkten auf einem Kegelschnitt, so dass sechszehn mal alle Berïhrungspunkte auf vier solchen Kegelschnitten liegen. Diese sechszehn Gruppirungen der sechszehn Kegelschnitte zu vieren sind folgende: 
$00,01,12,31 . \quad 20,21,10,33 . \quad 02,03,32,11 . \quad 22,23,30,13$. $20,21,12,31 . \quad 02,03,10,33 . \quad 22,23,32,11 . \quad 00,01,30,13$. $02,03,12,31 . \quad 22,23,10,33 . \quad 00,01,32,11 . \quad 20,21,30,13$. $22,23,12,31 . \quad 00,01,10,33 . \quad 20,21,32,11 . \quad 02,03,30,13$. $02,01,13,32 . \quad 22,21,11,30 . \quad 00,03,33,12 . \quad 20,23,31,10$. $22,21,13,32 . \quad 00,03,11,30 . \quad 20,23,33,12 . \quad 02,01,31,10$. $00,03,13,32 . \quad 20,23,11,30 . \quad 02,01,33,12 . \quad 22,21,31,10$. $20,23,13,32 . \quad 02,01,11,30 . \quad 22,21,33,12 . \quad 00,03,31,10$. $10,11,22,01 . \quad 30,31,20,03 . \quad 12,13,02,21 . \quad 32,33,00,23$. $30,31,22,01 . \quad 12,13,20,03 . \quad 32,33,02,21 . \quad 32,33,00,23$. $12,13,22,01 . \quad 32,33,20,03 . \quad 10,11,02,21 . \quad 10,11,00,23$. $32,33,22,01 . \quad 10,11,20,03 . \quad 30,31,02,21 . \quad 30,31,00,23$. $11,12,23,02 . \quad 31,32,21,00 . \quad 13,10,03,22 . \quad 33,30,01,20$. $31,32,23,02 . \quad 13,10,21,00 . \quad 33,30,03,22 . \quad 11,12,01,20$. $13,10,23,02 . \quad 33,30,21,00 . \quad 11,12,03,22 . \quad 31,32,01,20$. $33,30,23,02 . \quad 11,12,21,00$. 31, 32, 03, 22. 13, 10, 01, 20.

Im Ganzen also liegen $64+48+4=116$ mal vier Berïhrungspunkte mit den Doppelpunkten auf einem Kegelschnitt.

Man kann diese Sätze leicht fortentwickeln, namentlich auch in Bezng auf die Punktreihen, welche die Verbindungslinien der Berührungspunkte auf der Verbindungslinie der Doppelpunkte erzeugen.

Ich bemerke nur noch, dass die Formel (75.) sofort diejenigen Sätze ergiebt, die Steiner im $32^{\text {sten }}$ Bande pag. 184 dieses Journals angedeutet hat.

\$. 21.

Schnittcurven, welche durch einen Doppelpunkt gehen. Tangenten von einem Doppelpunkt an die Curve.

Die Gleichung (74.), welche zwischen den Argumenten der Schnittpunkte für den Fall besteht, dass die Curve $m^{\text {ter }}$ Ordnung durch einen der Doppelpunkte $\left(x_{1}=0, x_{2}=0\right)$ hindurchgeht, führt, wenn alle Argumente bis auf zwei gegeben sind, auf das in folgenden Gleichungen enthaltenen Umkehrungsproblem:

$\left(77^{a}.\right)$

$$
\left\{\begin{array}{c}
v_{1}+v_{2}=\omega, \\
\frac{H\left(\frac{\zeta+\sigma}{2}+\delta_{2}-v_{1}\right)}{H\left(\frac{\zeta+\sigma}{2}-\delta_{2}-v_{1}\right)} \cdot \frac{H\left(\frac{\zeta+\sigma}{2}+\delta_{2}-v_{2}\right)}{H\left(\frac{\zeta+\sigma}{2}-\delta_{2}-v_{2}\right)}=e^{\omega_{1}} .
\end{array}\right.
$$


Man kann dies Problem so lösen, dass man

(78.) $\quad v_{1}=\frac{1}{2} \omega+t, \quad v_{2}=\frac{1}{2} \omega-t$

setzt, und nun $t$ bestimmt. Die erste Gleichung ist hiedurch identisch erfüllt, die zweite giebt:

$$
\frac{H\left(\frac{\zeta+\sigma-\omega}{2}+\delta_{2}-t\right) H\left(\frac{\zeta+\sigma-\omega}{2}+\delta_{2}+t\right)}{H\left(\frac{\zeta+\sigma-\omega}{2}-\delta_{2}-t\right) H\left(\frac{\zeta+\sigma-\omega}{2}-\delta_{2}+t\right)}=e^{\omega_{1}},
$$

oder, nach bekannten Formẹln:

$$
\frac{\sin ^{2} \operatorname{am}\left(\frac{\zeta+\sigma-\omega}{2}+\delta_{2}\right)-\sin ^{2} \mathrm{am} t}{\sin ^{2} \operatorname{am}\left(\frac{\zeta+\sigma-\omega}{2}-\delta_{2}\right)-\sin ^{2} \operatorname{am} t}=e^{\omega_{1}} \cdot \frac{\Theta^{2}\left(\frac{\zeta+\sigma-\omega}{2}-\delta_{2}\right)}{\Theta^{2}\left(\frac{\zeta+\sigma-\omega}{2}+\delta_{2}\right)},
$$

und durch Auflösung findet man also:

$$
\text { (79.) } \sin ^{2} \mathrm{am} t=\frac{1}{k} \cdot \frac{H^{2}\left(\frac{\zeta+\sigma-\omega}{2}+\delta_{2}\right)-e^{\omega_{1}} H^{2}\left(\frac{\zeta+\sigma-\omega}{2}-\delta_{2}\right)}{\Theta^{2}\left(\frac{\zeta+\sigma-\omega}{2}+\delta_{2}\right)-e^{\omega_{1}} \Theta^{2}\left(\frac{\zeta+\sigma-\omega}{2}-\delta_{2}\right)} \text {. }
$$

Diese Gleichung in Verbindung mit (78.) enthält die vollständige Lösung der Aufgabe. Ist also in (74.) $m>n-3$, so findet man zu $4 m-3$ gegebenen Punkten aus (74.), (77 $\left.{ }^{a}\right)$, (78.), (79.) den $4 m-2^{\text {ten }}$ Punkt, der mit jenen und mit dem einen Doppelpunkte $\left(x_{2}=0, x_{1}=0\right)$ auf einer Curve $m^{\text {ter }}$ Ordnung liegt.

Der Fall $m=n-3$ bezieht sich hier auf die durch diesen Doppelpunkt gehenden Geraden. Für sie gehen die Gleichungen (74.), wenn $v_{1}, v_{2}$ sich auf die weiteren Schnittpunkte einer solchen Geraden mit der Curve beziehen, über in:

$$
\begin{gathered}
v_{1}+v_{2}=\zeta+\sigma, \\
\frac{H\left(\frac{\zeta+\sigma}{2}+\delta_{2}-v_{1}\right) H\left(\frac{\zeta+\sigma}{2}+\delta_{2}-v_{2}\right)}{H\left(\frac{\zeta+\sigma}{2}-\delta_{2}-v_{2}\right) H\left(\frac{\zeta+\sigma}{2}-\delta_{2}-v_{2}\right)}=1
\end{gathered}
$$

Erfüllt man die erste Gleichung durch die Annahme

$$
\text { (80.) } \quad v_{1}=\frac{\zeta+\sigma}{2}+t, \quad v_{2}=\frac{\zeta+\sigma}{2}-t,
$$

so wird die zweite identisch erfüllt, und die Gleichungen (80.) stellen also die allgemeinsten Argumente zweier Punkte dar, die mit dem betreffenden Doppelpunkte in einer Geraden liegen. 
Soll insbesondere die Gerade eine von dem Doppelpunkte gezogene Tangente werden, so dürfen $v_{1}, v_{2}$ sich nur um eine Periode unterscheiden, d. h. $t$ muss einen der Werthe $0, K, i K^{\prime}, K+i K^{\prime}$ annehmen. Von einem Doppelpunkte kann man also vier Tangenten an die Carve legen. Die vier Argumente derselben sind für den hier behandelten Doppelpunkt

$$
\frac{\zeta+\sigma}{2}, \frac{\zeta+\sigma}{2}+K, \quad \frac{\zeta+\sigma}{2}+i K^{\prime}, \quad \frac{\zeta+\sigma}{2}+K+i K^{\prime} .
$$

Die Argumente der dem anderen Doppelpunkte angehörigen Tangenten sind

$$
-\frac{\zeta+\sigma}{2},-\frac{\zeta+\sigma}{2}+K, \quad-\frac{\zeta+\sigma}{2}+i K^{\prime}, \quad-\frac{\zeta+\sigma}{2}+K+i K^{\prime} .
$$

Diese vier Tangenten entsprechen einzeln den ersten, so dass die Berihrungspunkte je zweier entsprechenden Paare mit den beiden Doppelpunkten auf einem Kegelschnitt liegen.

\section{§. 22.}

Allgemeine Schnittcurven. Bestimmung dreier Schnittpunkte aus den übrigen.

Die allgemeinen Bedingungen für das Schnittpunktsystem einer Curve $m^{\text {ter }}$ Ordnung mit der gegebenen Curve vierter Ordnung, welche in den Gleichungen (73.) enthalten sind, bestimmen drei Punkte des Systems durch die übrigen. Die Auffindung der drei Argumente dieser Punkte führt dann auf die Gleichungen :

$$
\begin{gathered}
v_{1}+v_{2}+v_{3}=\omega, \\
\frac{H\left(\frac{\zeta+\sigma}{2}+\delta_{2}-v_{1}\right) H\left(\frac{\zeta+\sigma}{2}+\delta_{2}-v_{2}\right) H\left(\frac{\zeta+\sigma}{2}+\delta_{2}-v_{3}\right)}{H\left(\frac{\zeta+\sigma}{2}-\delta_{2}-v_{1}\right) H\left(\frac{\zeta+\sigma}{2}-\delta_{2}-v_{2}\right) H\left(\frac{\zeta+\sigma}{2}-\delta_{2}-v_{3}\right)}=e^{\omega_{2}}, \\
\frac{H\left(-\frac{\zeta+\sigma}{2}+\delta_{3}-v_{1}\right) H\left(-\frac{\zeta+\sigma}{2}+\delta_{3}-v_{2}\right) H\left(-\frac{\zeta+\sigma}{2}+\delta_{3}-v_{3}\right)}{H\left(-\frac{\zeta+\sigma}{2}-\delta_{3}-v_{1}\right) H\left(-\frac{\zeta+\sigma}{2}-\delta_{3}-v_{2}\right) H\left(-\frac{\zeta+\sigma}{2}-\delta_{3}-v_{3}\right)}=e^{\omega_{3}} .
\end{gathered}
$$

Die Betrachtungen der \$\$. 10, 11 gestatten eine doppelte Lösung dieser Aufgabe. Erstlich kann man setzen

$$
z_{i}=\sin ^{2} \mathrm{am}\left(v_{i}-\frac{\omega}{3}\right),
$$


262 Clebsch, ebene Curven, deren Coord. elliptische Funct. eines Parameters sind.

und die drei $z$ bestimmen sich dann aus der Gleichung:

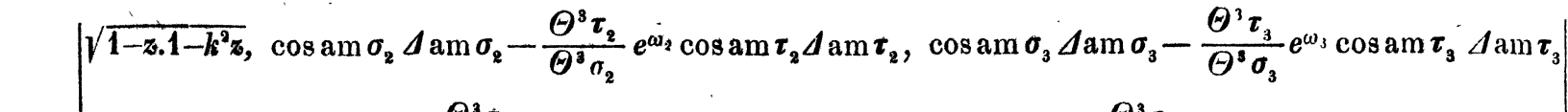

$$
\begin{aligned}
& 0=\sqrt{z}, \quad \sin a m \sigma_{2}-\frac{\Theta^{3} t_{2}}{\Theta^{3} \sigma_{2}} e^{\left(\omega_{2}\right.} \operatorname{sinam} \tau_{2} \\
& \sin \operatorname{arn} \sigma_{3}-\frac{\Theta^{3} \tau_{3}}{\Theta^{3} \sigma_{3}} e^{\omega_{3}} \sin \operatorname{am} \tau_{3} \\
& z \sqrt{z} \\
& \sin ^{3} \mathrm{am} \sigma_{2}-\frac{\Theta^{3} \tau_{2}}{\Theta^{3} \sigma_{2}} e^{\omega_{2}} \sin ^{3} \mathrm{am} \tau_{2}, \\
& \sin ^{3} \operatorname{am}_{3}-\frac{\Theta^{3} \tau_{3}}{\Theta^{3} \sigma_{3}} e^{\omega_{3}} \sin ^{3} \operatorname{am} \tau_{3}
\end{aligned}
$$

wo

$$
\begin{aligned}
& \sigma_{2}=\frac{\zeta+\sigma}{2}+\delta_{2}-\frac{\omega}{3}, \quad \sigma_{3}=-\frac{\zeta+\sigma}{2}+\delta_{3}-\frac{\omega}{3}, \\
& \tau_{2}=\frac{\zeta+\sigma}{2}-\delta_{2}-\frac{\omega}{3}, \quad \tau_{3}=-\frac{\zeta+\sigma}{2}-\delta_{3}-\frac{\omega}{3} .
\end{aligned}
$$

Zweitens kann man die Functionen einführen:

$$
\begin{aligned}
\Omega(u) & =\quad \boldsymbol{H}\left(u+\delta_{2}+\delta_{3}-\omega\right) \boldsymbol{H}\left(u-\frac{\zeta+\sigma}{2}-\delta_{2}\right) \boldsymbol{H}\left(u+\frac{\zeta+\sigma}{2}-\delta_{3}\right) \boldsymbol{H}\left(\zeta+\sigma+\delta_{2}-\delta_{3}\right) \\
& -e^{\omega_{2}} \quad \boldsymbol{H}\left(u-\delta_{2}+\delta_{3}-\omega\right) \boldsymbol{H}\left(u-\frac{\zeta+\sigma}{2}+\delta_{2}\right) \boldsymbol{H}\left(u+\frac{\zeta+\sigma}{2}-\delta_{3}\right) \boldsymbol{H}\left(\zeta+\sigma-\delta_{2}-\delta_{3}\right) \\
& -e^{\omega_{3}} \quad \boldsymbol{H}\left(u+\delta_{2}-\delta_{3}-\omega\right) \boldsymbol{H}\left(u-\frac{\zeta+\sigma}{2}-\delta_{2}\right) \boldsymbol{H}\left(u+\frac{\zeta+\sigma}{2}+\delta_{3}\right) \boldsymbol{H}\left(\zeta+\sigma+\delta_{2}+\delta_{3}\right) \\
& +e^{\omega_{2} \omega_{3}} \boldsymbol{H}\left(u-\delta_{2}-\delta_{3}-\omega\right) \boldsymbol{H}\left(u-\frac{\zeta+\sigma}{2}+\delta_{2}\right) \boldsymbol{H}\left(u+\frac{\zeta+\sigma}{2}+\delta_{3}\right) \boldsymbol{H}\left(\zeta+\sigma-\delta_{2}+\delta_{3}\right),
\end{aligned}
$$

und findet nach 41 :

$$
\begin{aligned}
\sqrt{z_{1} z_{2} z_{3}} & =\frac{i}{(\sqrt{ } k \sqrt{ } q)^{3}} \cdot e^{\frac{i \pi \omega}{2 K}} \frac{\Omega(o)}{\Omega\left(i K^{\prime}\right)}, \\
\sqrt{1-z_{1} \cdot 1-z_{2} \cdot 1-z_{3}} & =-\frac{i \sqrt{ } k^{\prime 3}}{(\sqrt{ } k \sqrt{ } q)^{3}} \cdot e^{\frac{i \pi \omega}{2 K}} \frac{\Omega(K)}{\Omega\left(i K^{\prime}\right)}, \\
\sqrt{1-k^{2} z_{1} \cdot 1-k^{2} z_{2} \cdot 1-k^{2} z_{3}} & =-i \sqrt{ } k^{\prime 3} \cdot e^{\frac{i \pi \omega}{2 K}} \frac{\Omega\left(K+i K^{\prime}\right)}{\Omega\left(i K^{\prime}\right)} .
\end{aligned}
$$

Diese Gleichungen gestatten sofort, wenn $m>1$, das Schnittpunktsystem zu vervollständigen. 


\section{\$. 23.}

Schnitt einer Geraden mit der Curve.

Für $m=1$ müssen, da zwei Schnittpunkte einer Geraden mit der Curve beliebig angenommen werden können, die Gleichungen des Abelschen Theorems (73.)

(81.)

$$
\left\{\begin{array}{c}
v_{1}+v_{2}+v_{3}+v_{4}=0, \\
\prod_{i=1}^{i=4} \frac{H\left(\frac{\zeta+\sigma}{2}+\delta_{2}-v_{i}\right)}{H\left(\frac{\zeta+\sigma}{2}-\delta_{2}-v_{i}\right)}=\frac{H\left(\zeta+\sigma+\delta_{2}+\delta_{3}\right) H\left(\zeta+\sigma+\delta_{2}-\delta_{3}\right)}{H\left(\zeta+\sigma-\delta_{2}-\delta_{3}\right) H\left(\zeta+\sigma-\delta_{2}+\delta_{3}\right)}, \\
\prod_{i=1}^{i=4} \frac{H\left(\frac{\zeta+\sigma}{2}+\delta_{3}-v_{i}\right)}{H\left(\frac{\zeta+\sigma}{2}-\delta_{3}-v_{i}\right)}=\frac{H\left(\zeta+\sigma-\delta_{2}-\delta_{3}\right) H\left(\zeta+\sigma+\delta_{2}-\delta_{3}\right)}{H\left(\zeta+\sigma+\delta_{2}+\delta_{3}\right) H\left(\zeta+\sigma-\delta_{2}+\delta_{3}\right)}
\end{array}\right.
$$

sich auf nur zwei reduciren, d. h. es müssen die beiden letzten Gleichungen mit Hülfe der ersten sich in eine einzige überführen lassen. Ich werde zeigen, dass sich die zweite Gleichung wirklich mit Hülfe der ersten in eine Gestalt bringen lässt, welche sich nicht mehr ändert, wenn $\zeta+\sigma$ mit $-(\zeta+\sigma)$ und $\delta_{2}$ mit $\delta_{3}$ vertauscht wird, wodurch die zweite Gleichung in die dritte übergeht. Hierzu bediene ich mich der Relationen zwischen verschiedenen $\boldsymbol{H}$ und $\Theta$, welche von Jacobi herrühren, und welche Rosenhain in der schon öfters angeführten Abhandlung vollständig bekannt gemacht hat. Sind $u, u^{\prime}, u^{\prime \prime}, u^{\prime \prime \prime}$ beliebige Argumente, und setzt man

$$
\begin{aligned}
& 2 \omega=u+u^{\prime}+u^{\prime \prime}+u^{\prime \prime \prime}, \\
& 2 \omega^{\prime}=u+u^{\prime}-u^{\prime \prime}-u^{\prime \prime \prime}, \\
& 2 \omega^{\prime \prime}=u-u^{\prime}+u^{\prime \prime}-u^{\prime \prime \prime}, \\
& 2 \omega^{\prime \prime \prime}=u-u^{\prime}-u^{\prime \prime}+u^{\prime \prime \prime},
\end{aligned}
$$

so ist die hier zur Benutzung kommende Gleichung:

$$
\begin{gathered}
2 \boldsymbol{H}(u) \boldsymbol{H}\left(u^{\prime}\right) \boldsymbol{H}\left(u^{\prime \prime}\right) \boldsymbol{H}\left(u^{\prime \prime \prime}\right) \\
=\boldsymbol{H}(\omega) \boldsymbol{H}\left(\omega^{\prime}\right) \boldsymbol{H}\left(\omega^{\prime \prime}\right) \boldsymbol{H}\left(\omega^{\prime \prime \prime}\right)-\boldsymbol{H}(\omega+\boldsymbol{K}) \boldsymbol{H}\left(\omega^{\prime}+\boldsymbol{K}\right) \boldsymbol{H}\left(\omega^{\prime \prime}+\boldsymbol{K}\right) \boldsymbol{H}\left(\omega^{\prime \prime \prime}+\boldsymbol{K}\right) \\
-\Theta(\omega) \Theta\left(\omega^{\prime}\right) \Theta\left(\omega^{\prime \prime}\right) \Theta\left(\omega^{\prime \prime \prime}\right)+\Theta(\omega+\boldsymbol{K}) \Theta\left(\omega^{\prime}+\boldsymbol{K}\right) \Theta\left(\omega^{\prime \prime}+\boldsymbol{K}\right) \Theta\left(\omega^{\prime \prime \prime}+\boldsymbol{K}\right) .
\end{gathered}
$$

Mit Hülfe dieser Formel kann man Zähler und Nenner der zweiten Gleichung (81.) nmgestalten. Die $v$ gehen dann immer aus $w$ heraus, weil ihre Summe verschwindet; dagegen werden umgekehrt $\omega^{\prime}, \omega^{\prime \prime}, \omega^{\prime \prime \prime}$ immer von den constanten Theilen der Argumente unabhängig, und nehmen in Zähler und Nenner die- 
selben Werthe an. Dividirt man dann, nachdem die Nenner hinaufmultiplicirt sind, mit $\Theta\left(\omega^{\prime}\right) \cdot \Theta\left(\omega^{\prime \prime}\right) \cdot \Theta\left(\omega^{\prime \prime \prime}\right)$, und setzt

$$
\text { (82.) }\left\{\begin{array}{l}
x^{\prime}=\sin ^{2} \mathrm{am}\left(\omega^{\prime}\right)=\sin ^{2} \mathrm{am} \frac{v_{1}+v_{2}-v_{3}-v_{4}}{2}, \\
x^{\prime \prime}=\sin ^{2} \mathrm{am}\left(\omega^{\prime \prime}\right)=\sin ^{2} \mathrm{am} \frac{v_{1}-v_{2}+v_{3}-v_{4}}{2}, \\
x^{\prime \prime \prime}=\sin ^{2} \mathrm{am}\left(\omega^{\prime \prime \prime}\right)=\sin ^{2} \mathrm{am} \frac{v_{1}-v_{2}-v_{3}+v_{4}}{2},
\end{array}\right.
$$

so geht die zweite Gleichung (81.) über in:

(83.) $0=A+B \cdot \sqrt{x^{\prime} x^{\prime \prime} x^{\prime \prime \prime}}+C \sqrt{1-x^{\prime} .1-x^{\prime \prime} .1-x^{\prime \prime \prime}}+D \sqrt{1-k^{2} x^{\prime} .1-k^{2} x^{\prime \prime} .1-k^{2}} x^{\prime \prime \prime}$.

Und zwar ist, wenn der Kürze wegen $\zeta+\sigma=\tau$ gesetzt wird:

$$
\begin{aligned}
-A= & \Theta\left(\tau+2 \delta_{2}\right) H\left(\tau-\delta_{2}-\delta_{3}\right) H\left(\tau-\delta_{2}+\delta_{3}\right)-\Theta\left(\tau-2 \delta_{2}\right) H\left(\tau+\delta_{2}+\delta_{3}\right) H\left(\tau+\delta_{2}-\delta_{3}\right), \\
\frac{B}{\sqrt{k^{3}}}=H\left(\tau+2 \delta_{2}\right) H\left(\tau-\delta_{2}-\delta_{3}\right) H\left(\tau-\delta_{2}+\delta_{3}\right)-H\left(\tau-2 \delta_{2}\right) H\left(\tau+\delta_{2}+\delta_{3}\right) H\left(\tau+\delta_{2}-\delta_{3}\right), & \\
-C \sqrt{\frac{k^{\prime}}{k}}=H\left(\tau+2 \delta_{2}+K\right) H\left(\tau-\delta_{2}-\delta_{3}\right) H\left(\tau-\delta_{2}+\delta_{3}\right) & -H\left(\tau-2 \delta_{2}+K\right) H\left(\tau+\delta_{2}+\delta_{3}\right) H\left(\tau+\delta_{2}-\delta_{3}\right), \\
D \sqrt{\bar{k}^{3}}=\Theta\left(\tau+2 \delta_{2}+K\right) H\left(\tau-\delta_{2}-\delta_{3}\right) H\left(\tau-\delta_{2}+\delta_{3}\right) & -\Theta\left(\tau-2 \delta_{2}+K\right) H\left(\tau+\delta_{2}+\delta_{3}\right) H\left(\tau+\delta_{2}-\delta_{3}\right) .
\end{aligned}
$$

Man kann leicht aus einem dieser Coefficienten die übrigen ableiten. Ist nämlich

$$
\frac{B}{\sqrt{k}^{3}}=f\left(\tau, \delta_{2}, \delta_{3}\right)
$$

so ist auch

$$
\begin{aligned}
A & =i q \sqrt[4]{q} e^{\frac{i \pi}{2 K}\left(3 \tau+2 \delta_{3}\right)} \cdot f\left(\tau+i K^{\prime}, \delta_{2}, \delta_{3}+i K^{\prime}\right), \\
C \sqrt{\frac{\bar{k}^{\prime}}{k}} & =f\left(\tau+K, \delta_{2}, \delta_{3}+K\right), \\
D \bar{V}^{3} & =-q \sqrt{q} e^{\frac{i \pi}{2 K}\left(3 \tau+2 \delta_{3}\right)} \cdot f\left(\tau+K+i K^{\prime}, \delta_{2}, \delta_{3}+K+i K^{\prime}\right) .
\end{aligned}
$$

Es ist also nur einer dieser Coefficienten näher zu untersuchen. Man hat nach der vorhin benutzten Formel, indem man $\frac{B}{\sqrt{k^{3}}}$ mit $H(\tau)$ multiplicirt, jedes Product rechts transformirt, und die șich aufhebenden Terme auslässt:

$$
\frac{B}{\sqrt{ } k^{3}} \cdot \boldsymbol{H}(\tau)=\boldsymbol{H}(2 \tau) \boldsymbol{H}\left(2 \delta_{2}\right) \boldsymbol{H}\left(\delta_{2}+\delta_{3}\right) \boldsymbol{H}\left(\delta_{2}-\delta_{3}\right) \text {. }
$$


Daher hat man auch:

$$
\begin{aligned}
\boldsymbol{A} \cdot \Theta(\tau) & =\boldsymbol{H}(2 \tau) \boldsymbol{H}\left(2 \delta_{2}\right) \Theta\left(\delta_{2}+\delta_{3}\right) \Theta\left(\delta_{2}-\delta_{3}\right), \\
C \sqrt{\frac{k^{\prime}}{k}} \cdot \boldsymbol{H}(\tau+\boldsymbol{K}) & =\boldsymbol{H}(2 \tau) \boldsymbol{H}\left(2 \delta_{2}\right) \boldsymbol{H}\left(\delta_{2}+\delta_{3}+\boldsymbol{K}\right) \boldsymbol{H}\left(\delta_{2}-\delta_{3}+\boldsymbol{K}\right), \\
\boldsymbol{D}_{{\sqrt{k^{\prime}}}^{3}} \cdot \Theta(\tau+\boldsymbol{K}) & =-\boldsymbol{H}(2 \tau) \boldsymbol{H}\left(2 \delta_{2}\right) \Theta\left(\delta_{2}+\delta_{3}+\boldsymbol{K}\right) \Theta\left(\delta_{2}-\delta_{3}+\boldsymbol{K}\right),
\end{aligned}
$$

und die Gleichung (83.) geht in folgende bemerkenswerthe Form über:

$$
\begin{aligned}
0=1+k^{2} \sqrt{\frac{a b x^{\prime} x^{\prime \prime} x^{\prime \prime \prime}}{c}}+\frac{k^{2}}{k^{\prime 2}} \sqrt{\frac{1-a .1-b .1-x^{\prime} .1-x^{\prime \prime} .1-x^{\prime \prime \prime}}{1-c}} \\
-\frac{1}{k^{\prime 2}} \sqrt{\frac{1-k^{2} a .1-k^{2} b .1-k^{2} x^{\prime} .1-k^{2} x^{\prime \prime} .1-k^{2} x^{\prime \prime \prime}}{1-k^{2} c}}
\end{aligned}
$$

wo

$$
\begin{aligned}
& a=\sin ^{2} \operatorname{am}\left(\delta_{2}+\delta_{3}\right), \\
& b=\sin ^{2} \operatorname{am}\left(\delta_{2}-\delta_{3}\right), \\
& c=\sin ^{2} \operatorname{am}(\zeta+\sigma) .
\end{aligned}
$$

Diese Gleichung ändert sich durch Vertauschung von $\delta_{2}$ mit $\delta_{3}, \zeta+\sigma$ mit $-(\zeta+\sigma)$ nicht mehr; dieselbe Gleichung wird also auch aus der dritten Gleichung (81.) erhalten, und diese kann daher nichts Neues mehr liefern.

Man kann diese Resultate in folgendem Satz aussprechen:

Die allgemeinsten Argumente, welche das Schnittpunktsystem einer Geraden mit der Curve vierter Ordnung bilden können, sind

$$
v_{1}=\frac{\omega^{\prime}+\omega^{\prime \prime}+\omega^{\prime \prime \prime}}{2}, v_{2}=\frac{\omega^{\prime}-\omega^{\prime \prime}-\omega^{\prime \prime \prime}}{2}, v_{3}=\frac{-\omega^{\prime}+\omega^{\prime \prime}-\omega^{\prime \prime \prime}}{2}, v_{4}=\frac{-\omega^{\prime}-\omega^{\prime \prime}+\omega^{\prime \prime \prime}}{2} \text {, }
$$

wo $\omega, \omega^{\prime}, \omega^{\prime \prime}$ durch die eine Gleichung verbunden sind:

$$
\left\{\begin{array}{r}
0=1+k^{2} \frac{\sin a m \omega^{\prime} \sin a m \omega^{\prime \prime} \sin a m \omega^{\prime \prime \prime} \sin \operatorname{am}\left(\delta_{2}+\delta_{3}\right) \sin a m\left(\delta_{2}-\delta_{3}\right)}{\sin \operatorname{am}(\zeta+\sigma)} \\
+\frac{k^{2}}{k^{\prime 2}} \frac{\cos a m \omega^{\prime} \cos a m \omega^{\prime \prime} \cos a m \omega^{\prime \prime \prime} \cos a m\left(\delta_{2}+\delta_{3}\right) \cos a m\left(\delta_{2}-\delta_{3}\right)}{\cos a m(\zeta+\sigma)} \\
-\frac{1}{k^{\prime 2}} \frac{\Delta a m \omega^{\prime} \Delta \operatorname{am} \omega^{\prime \prime} \Delta \operatorname{am} \omega^{\prime \prime \prime} \Delta \operatorname{am}\left(\delta_{2}+\delta_{3}\right) \Delta \operatorname{am}\left(\delta_{2}-\delta_{3}\right)}{\Delta \operatorname{am}(\zeta+\sigma)} .
\end{array}\right.
$$

\section{\$. 24.}

Wendetangenten und Doppeltangenten.

Wenden wir diese Gleichung insbesondere auf die Wendetangenten und Doppeltangenten an. Für die Wendepunkte muss $\omega^{\prime}=\omega^{\prime \prime}=\omega^{\prime \prime \prime}$ sein, damit auch $v_{2}=v_{3}=v_{4}$ werde, und man erhält also sur Bestimmung der ArJournal für Mathematik Bd. LXIV. Heft 3. 
gumente $v=-\frac{\omega}{2}$ der, Wendepunkte die Gleichung:

$$
\begin{aligned}
0=1 & +k^{2} \frac{\sin ^{3} \mathrm{am} \omega \sin \operatorname{am}\left(\delta_{2}+\delta_{3}\right) \sin \operatorname{am}\left(\delta_{2}-\delta_{3}\right)}{\sin \mathrm{am}(\zeta+\sigma)} \\
& +\frac{k^{2}}{k^{\prime 2}} \frac{\cos ^{3} \mathrm{am} \omega \cos a m\left(\delta_{2}+\delta_{3}\right) \cos a m\left(\delta_{2}-\delta_{3}\right)}{\cos \mathrm{am}(\zeta+\sigma)} \\
& -\frac{1}{k^{\prime 2}} \frac{\Delta^{3} \mathrm{am} \omega \Delta \operatorname{am}\left(\delta_{2}+\delta_{3}\right) \Delta \mathrm{am}\left(\delta_{2}-\delta_{3}\right)}{\Delta(\zeta+\sigma)},
\end{aligned}
$$

welche, wenn man sie in eine rationale Gleichung für sin amw verwandelt, vom zwölften Grade wird, den zwölf verschiedenen Wendepunkten entsprechend. Ich bemerke, dass $\omega$ sich aus dieser Gleichung bis auf Vielfache von $4 K+4 i K^{\prime}$, also $v=-\frac{\omega}{2}$ bis auf Vielfache von $2 K+2 i K^{\prime}$ bestimmt, und somit die zugehörigen Coordinaten eindeutig gegeben werden.

Sollen die Argumente $v$ einer Doppeltangente entsprechen, und zwar $v_{1}, v_{2}$ einem Berührungspunkte, $v_{3}, v_{4}$ dem andern, so muss sowohl $\omega^{\prime \prime}+\omega^{\prime \prime \prime}$ als $\omega^{\prime \prime}-\omega^{\prime \prime \prime}$ von der Form $2 p K+2 q i K^{\prime}$ sein, d. h. man kann setzen

$$
\omega^{\prime \prime}=\omega^{\prime \prime \prime}=p K+q i K^{\prime} \text {. }
$$

Nach den Werthen von $p, q$ sondern sich also die acht Doppeltangenten in vier Paare; und die zugehörigen Werthe von $\omega^{\prime}$ bestimmen sich aus den Gleichungen:

I) $p=0, q=0$ :

$$
\begin{aligned}
0=1+\frac{k^{2}}{k^{\prime 2}} \frac{\operatorname{cosam} \omega^{\prime} \cos \operatorname{am}\left(\delta_{2}+\delta_{3}\right) \operatorname{cosam}\left(\delta_{2}-\delta_{3}\right)}{\operatorname{cosam}(\zeta+\sigma)} \\
-\frac{1}{k^{\prime 2}} \frac{\Delta \operatorname{am} \omega^{\prime} \Delta \operatorname{am}\left(\delta_{2}+\delta_{3}\right) \Delta \operatorname{am}\left(\delta_{2}-\delta_{3}\right)}{\Delta \operatorname{am}(\zeta+\sigma)},
\end{aligned}
$$

II) $p=1, q=0$ :

$$
\begin{aligned}
& 0=1+k^{2} \frac{\sin a m \omega^{\prime} \sin a m\left(\delta_{2}+\delta_{3}\right) \sin a m\left(\delta_{2}-\delta_{3}\right)}{\sin \operatorname{am}(\zeta+\sigma)} \\
& -\frac{\Delta \mathrm{am} \omega^{\prime} \Delta \mathrm{am}\left(\delta_{2}+\delta_{8}\right) \Delta \mathrm{am}\left(\delta_{2}-\delta_{3}\right)}{\Delta \operatorname{am}(\zeta+\sigma)},
\end{aligned}
$$

III) $p=1, q=1$ :

$$
\begin{aligned}
& 0=1+\frac{\sin a m \omega^{\prime} \sin a m\left(\delta_{2}+\delta_{3}\right) \sin a m\left(\delta_{2}-\delta_{3}\right)}{\sin a m(\xi+\sigma)} \\
& +\frac{\cos a m \omega^{\prime} \cos a m\left(\delta_{2}+\delta_{3}\right) \cos a m\left(\delta_{2}-\delta_{3}\right)}{\cos a m(\zeta+\sigma)},
\end{aligned}
$$

IV) $p=0, \quad q=1$ :

$$
\begin{aligned}
0 & =\frac{\operatorname{sinam} \omega^{\prime} \sin \operatorname{am}\left(\delta_{2}+\delta_{3}\right) \sin \operatorname{am}\left(\delta_{2}-\delta_{3}\right)}{\sin \operatorname{am}(\zeta+\sigma)} \\
+ & \frac{k^{2}}{k^{\prime 2}} \frac{\operatorname{cosam} \omega^{\prime} \operatorname{cosam}\left(\delta_{2}+\delta_{3}\right) \operatorname{cosam}\left(\delta_{2}-\delta_{3}\right)}{\cos \operatorname{am}(\zeta+\sigma)}-\frac{1}{k^{\prime 2}} \frac{\Delta \operatorname{sam} \omega^{\prime} \Delta \operatorname{am}\left(\delta_{3}+\delta_{3}\right) \Delta a m\left(\delta_{2}-\delta_{3}\right)}{\Delta \operatorname{am}(\zeta+\sigma)} .
\end{aligned}
$$


Vergleicht man diese Gleichungen mit den folgenden vier Formen des Additionstheorems :

$\Delta \operatorname{am} \alpha \Delta \operatorname{am} \beta \Delta \operatorname{am}(\alpha \pm \beta)-k^{2} \cos \operatorname{am} \alpha \cos \operatorname{am} \beta \cos \operatorname{am}(\alpha \pm \beta)=k^{\prime 2}$,

$\frac{\Delta \operatorname{am} \alpha \Delta \operatorname{am} \beta \Delta \operatorname{am}(\alpha \pm \beta \pm K)}{k^{\prime}}-k^{2} \sin \operatorname{am} \alpha \sin \operatorname{am} \beta \sin \operatorname{am}(\alpha \pm \beta \pm K)=1$,

$\frac{i k}{k^{\prime}} \operatorname{cosam} \alpha \operatorname{cosam} \beta \operatorname{cosam}\left(\alpha \pm \beta \pm K \pm i K^{\prime}\right)-k \sin a m \alpha \sin a m \beta \operatorname{sinam}\left(\alpha \pm \beta \pm K \pm i K^{\prime}\right)=1$,

$\cos \operatorname{am} \alpha \cos a m \beta \cos a m\left(\alpha \pm \beta \pm i K^{\prime}\right)+i k^{\prime 2} \sin \operatorname{am} \alpha \sin \operatorname{am} \beta \sin$ am $\left(\alpha \pm \beta \pm i K^{\prime}\right)$

$$
-\frac{\Delta \operatorname{am} \alpha \Delta \operatorname{am} \beta \Delta \operatorname{am}\left(\alpha \pm \beta \pm i K^{\prime}\right)}{k}=0,
$$

so findet man für die obigen vier Gleichungen folgende Auflösungen:

I. Man setze

$$
\begin{gathered}
\cos \operatorname{am} \alpha \cos \operatorname{am} \beta=\frac{\operatorname{cosam}\left(\delta_{2}+\delta_{3}\right) \operatorname{cosam}\left(\delta_{2}-\delta_{3}\right)}{\cos \operatorname{am}(\zeta+\sigma)}, \\
\Delta \operatorname{am} \alpha \Delta \operatorname{am} \beta=\frac{\Delta \operatorname{am}\left(\delta_{2}+\delta_{3}\right) \Delta \operatorname{am}\left(\delta_{2}-\delta_{3}\right)}{\Delta \operatorname{am}(\zeta+\sigma)}, \\
\omega^{\prime}=\alpha+\beta, \text { oder } \omega^{\prime}=\alpha-\beta .
\end{gathered}
$$

II. Man setze

$$
\begin{gathered}
\sin \operatorname{am} \alpha^{\prime} \sin \operatorname{am} \beta^{\prime}=\frac{\sin \operatorname{am}\left(\delta_{2}+\delta_{3}\right) \sin \operatorname{am}\left(\delta_{2}-\delta_{3}\right)}{\sin \operatorname{am}(\zeta+\sigma)} \\
\Delta \operatorname{am} \alpha^{\prime} \Delta \mathrm{am} \beta^{\prime}=k^{\prime} \frac{\Delta \operatorname{am}\left(\delta_{2}+\delta_{3}\right) \Delta \mathrm{am}\left(\delta_{2}-\delta_{3}\right)}{\Delta \operatorname{am}(\zeta+\sigma)}, \\
\omega^{\prime}=\alpha^{\prime}+\beta^{\prime}+K, \text { oder } \omega^{\prime}=\alpha^{\prime}-\beta^{\prime}-K
\end{gathered}
$$

III. Man setze

$$
\begin{aligned}
& \sin a m \alpha^{\prime \prime} \sin a m \beta^{\prime \prime}=\frac{1}{k} \cdot \frac{\sin a m\left(\delta_{2}+\delta_{3}\right) \sin a m\left(\delta_{2}-\delta_{3}\right)}{\sin a m(\zeta+\sigma)} \\
& \cos a m \alpha^{\prime \prime} \cos a m \beta^{\prime \prime}=\frac{i k^{\prime}}{k} \cdot \frac{\cos a m\left(\delta_{2}+\delta_{3}\right) \cos a m\left(\delta_{2}-\delta_{3}\right)}{\cos a m(\zeta+\sigma)} \\
& \omega^{\prime}=\alpha^{\prime \prime}+\beta^{\prime \prime}+K+i K \text { oder } \omega^{\prime}=\alpha^{\prime \prime}-\beta^{\prime \prime}-K-i K^{\prime}
\end{aligned}
$$

IV. Man setze

$$
\begin{gathered}
\frac{\sin \operatorname{am} \alpha^{\prime \prime \prime} \sin \operatorname{am} \beta^{\prime \prime \prime}}{\Delta \operatorname{am} \alpha^{\prime \prime \prime} \Delta \operatorname{am} \beta^{\prime \prime \prime}}=\frac{i}{k^{\prime}} \cdot \frac{\sin \operatorname{am}\left(\delta_{2}+\delta_{3}\right) \sin \operatorname{am}\left(\delta_{2}-\delta_{3}\right) \Delta \operatorname{am}(\zeta+\sigma)}{\Delta \operatorname{am}\left(\delta_{2}+\delta_{3}\right) \Delta \operatorname{am}\left(\delta_{2}-\delta_{3}\right) \sin \operatorname{am}(\zeta+\sigma)}, \\
\frac{\cos \operatorname{am} \alpha^{\prime \prime \prime} \operatorname{cosam} \beta^{\prime \prime \prime}}{\Delta \operatorname{am} \alpha^{\prime \prime \prime} \Delta \operatorname{am} \beta^{\prime \prime \prime}}=\frac{1}{k} \cdot \frac{\operatorname{cosam}\left(\delta_{2}+\delta_{3}\right) \operatorname{cosam}\left(\delta_{2}-\delta_{3}\right) \Delta \operatorname{am}(\zeta+\sigma)}{\Delta \operatorname{am}\left(\delta_{2}+\delta_{3}\right) \Delta \operatorname{am}\left(\delta_{2}-\delta_{3}\right) \operatorname{cosam}(\zeta+\sigma)}, \\
\omega^{\prime}=\alpha^{\prime \prime \prime}+\beta^{\prime \prime \prime}+i K^{\prime} \text { oder } \omega^{\prime}=\alpha^{\prime \prime \prime}-\beta^{\prime \prime \prime}-i K^{\prime} .
\end{gathered}
$$

Man erhält so also wirklich acht verschiedene Werthe von $\omega^{\prime}$, und in den 
268 Clebsch, ebene Curven, deren Coord elliptische Funct. eines Parameters sind.

Ausdrücken

$$
v_{1}=\frac{\omega^{\prime}}{2}+p K+q i K^{\prime}, \quad v_{2}=-\frac{\omega^{\prime}}{2}
$$

die Argumente der Berührungspunkte.

\$. 25.

- Andre Bestimmung der Doppeltangenten.

Für das Studium der gegenseitigen Lage dieser Punkte ist es aber zweckmässiger auf die Gleichungen (81.) zurückzugehen. Setzt man

$$
\begin{aligned}
& v_{i}^{\prime}=\log \left\{\frac{H\left(\frac{\zeta+\sigma}{2}+\delta_{2}-v_{i}\right)}{H\left(\frac{\zeta+\sigma}{2}-\delta_{2}-v_{i}\right)} \cdot \sqrt[4]{\frac{H\left(\zeta+\sigma-\delta_{2}-\delta_{3}\right) H\left(\zeta+\sigma-\delta_{2}+\delta_{3}\right)}{H\left(\zeta+\sigma+\delta_{2}+\delta_{3}\right) H\left(\zeta+\sigma+\delta_{2}-\delta_{3}\right)}}\right\}, \\
& v_{i}^{\prime \prime}=\log \left\{\frac{H\left(-\frac{\zeta+\sigma}{2}+\delta_{3}-v_{i}\right)}{H\left(-\frac{\zeta+\sigma}{2}-\delta_{3}-v_{i}\right)} \cdot \sqrt[4]{\frac{H\left(\zeta+\sigma+\delta_{2}+\delta_{3}\right) H\left(\zeta+\sigma-\delta_{2}+\delta_{3}\right)}{\bar{H}\left(\zeta+\sigma-\delta_{2}-\delta_{3}\right) H\left(\zeta+\sigma+\delta_{2}-\delta_{3}\right)}}\right\},
\end{aligned}
$$

so nehmen jene Gleichungen, mit Hinzufügung der Perioden, die Gestalt an:

$$
\begin{aligned}
& v_{1}+v_{2}+v_{3}+v_{4}=2 p K+2 q i K^{\prime}, \\
& v_{1}^{\prime}+v_{2}^{\prime}+v_{3}^{\prime}+v_{4}^{\prime}=2 h^{\prime} i \pi+\frac{2 q i \pi \delta_{2}}{K}, \\
& v_{1}^{\prime \prime}+v_{2}^{\prime \prime}+v_{3}^{\prime \prime}+v_{4}^{\prime \prime}=2 h^{\prime \prime} i \pi+\frac{2 q i \pi \delta_{3}}{K},
\end{aligned}
$$

Lässt man nun für eine Doppeltangente die $v$ paarweise gleich werden, und bezeichnet durch $v, v_{0}$ die Argumente der Berūhrungspunkte, so gehen diese Gleichungen über in

$$
\begin{aligned}
& v+v_{0}=p K+q i K^{\prime}, \\
& v^{\prime}+v_{0}^{\prime}=h^{\prime} i \pi+\frac{q i \pi \delta_{2}}{K}, \\
& v^{\prime \prime}+v_{0}^{\prime \prime}=h^{\prime \prime} i \pi+\frac{q i \pi \delta_{3}}{K} .
\end{aligned}
$$

Zwischen den ganzen Zahlen $p, q, h^{\prime}, h^{\prime \prime}$ besteht die in $\$$. 13. allgemein entwickelte Relation

$$
(q+1)(p+1)+h^{\prime}+h^{\prime \prime} \equiv 1(\bmod .2) .
$$

Man kann dieselbe für den vorliegenden Fall folgendermassen ableiten. Nach der bekannten Formel

$$
\boldsymbol{H}(u+v) H(u-v) \cdot \theta^{2}(0)=H^{2}(u) \theta^{2}(v)-\theta^{2}(u) H^{2}(v)
$$


hat man

$$
\begin{aligned}
& =\mu \cdot \frac{H^{2}\left(\frac{\zeta+\sigma}{2}+\delta_{2}-\frac{p K+q i K^{\prime}}{2}\right) \Theta^{2} \frac{e^{h i i \pi+\frac{q i \pi \delta_{2}}{K}}}{2}-H^{2} \frac{v-v_{0}}{2} \Theta^{2}\left(\frac{\zeta+\sigma}{2}+\delta_{2}-\frac{p K+q i K^{\prime}}{2}\right)}{H^{2}\left(\frac{\zeta+\sigma}{2}-\delta_{2}-\frac{p K+q i K^{\prime}}{2}\right) \Theta^{2} \frac{v-v_{0}}{2}-H^{2} \frac{v-v_{0}}{2} \Theta^{2}\left(\frac{\zeta+\sigma}{2}-\delta_{2}-\frac{p K+q i K^{\prime}}{2}\right)}, \\
& =\nu \cdot \frac{H^{2}\left(-\frac{\zeta+\sigma}{2}+\delta_{3}-\frac{p K+q i K^{\prime}}{2}\right) \Theta^{2} \frac{v-v_{0}}{2}-H^{2} \frac{v-v_{0}}{2} \Theta^{2}\left(-\frac{\zeta+\sigma}{2}+\delta_{3}-\frac{p K+q i K^{\prime}}{2}\right)}{H^{2}\left(-\frac{\zeta+\sigma}{2}-\delta_{3}-\frac{p K+q i K^{\prime}}{2}\right) \Theta^{2} \frac{v-v_{0}}{2}-H^{2} \frac{v-v_{0}}{2} \Theta^{2}\left(-\frac{\zeta+\sigma}{2}-\delta_{3}-\frac{p K+q i K^{\prime}}{2}\right)},
\end{aligned}
$$

wo

$$
\begin{aligned}
& \mu=\sqrt{\frac{H\left(\zeta+\sigma-\delta_{2}-\delta_{3}\right) H\left(\zeta+\sigma-\delta_{2}+\delta_{3}\right)}{H\left(\zeta+\sigma+\delta_{2}+\delta_{3}\right) H\left(\zeta+\sigma+\delta_{2}-\delta_{3}\right)}}, \\
& \nu=\sqrt{\frac{H\left(\zeta+\sigma+\delta_{2}+\delta_{3}\right) H\left(\zeta+\sigma-\delta_{2}+\delta_{3}\right)}{H\left(\zeta+\sigma-\delta_{2}-\delta_{3}\right) H\left(\zeta+\sigma+\delta_{2}-\delta_{3}\right)}} .
\end{aligned}
$$

Bestimmt man nun den Quotienten $\Theta^{2} \frac{v-v_{0}}{2}: H^{2} \frac{v-v_{0}}{2}$ aus beiden Gleichungen, so findet sich

$$
\begin{gathered}
\frac{H^{2} \frac{v-v_{0}}{2}}{\Theta^{2} \frac{v-v_{0}}{2}}=k \sin ^{2} \mathrm{am} \frac{v-v_{0}}{2} \\
=\frac{\mu H^{2}\left(\frac{\zeta+\sigma}{2}+\delta_{2}-\frac{p K+q i K^{\prime}}{2}\right)-H^{2}\left(\frac{\zeta+\sigma}{2}-\delta_{2}-\frac{p K+q i K^{\prime}}{2}\right) \cdot e^{h^{\prime} i \pi+\frac{q i n \delta_{2}}{K}}}{\mu \Theta^{2}\left(\frac{\zeta+\sigma}{2}+\delta_{2}-\frac{p K+q i K^{\prime}}{2}\right)-\Theta^{2}\left(\frac{\zeta+\sigma}{2}-\delta_{2}-\frac{p K+q i K^{\prime}}{2}\right) \cdot e^{h^{\prime} i \pi+\frac{q i \pi \delta_{2}}{K}}} \\
=\frac{\nu H^{2}\left(-\frac{\zeta+\sigma}{2}+\delta_{3}-\frac{p K+q i K^{\prime}}{2}\right)-H^{2}\left(-\frac{\zeta+\sigma}{2}-\delta_{3}-\frac{p K+q i K^{\prime}}{2}\right) \cdot e^{h^{\prime \prime \prime i n+}+\frac{q i \pi \delta_{3}}{K}}}{\nu \Theta^{2}\left(-\frac{\zeta+\sigma}{2}+\delta_{3}-\frac{p K+q i K^{\prime}}{2}\right)-\Theta^{2}\left(-\frac{\zeta+\sigma}{2}-\delta_{3}-\frac{p K+q i K^{\prime}}{2}\right) \cdot e^{h^{\prime \prime} i \pi+\frac{q i \pi \delta_{3}}{K}}} .
\end{gathered}
$$

Diese Gleichungen geben einerseits eine einfachere Bestimmung der Doppeltangente, indem $\frac{v-v_{0}}{2}$ direct gegeben wird. Andrerseits folgt aus der Vergleichung beider Ausdrücke, und mit Anwendung der obigen Formel:

$$
\begin{aligned}
& \mathbf{0}=\quad \mu \nu \boldsymbol{H}\left(\delta_{2}+\delta_{3}-p K-q i K^{\prime}\right) \boldsymbol{H}\left(\zeta+\sigma+\delta_{2}-\delta_{3}\right) \\
& -\mu e^{h^{\prime \prime i} i \pi+\frac{q i \pi \delta_{3}}{K}} H\left(\delta_{2}-\delta_{3}-p K-q i K^{\prime}\right) H\left(\zeta+\sigma+\delta_{2}+\delta_{3}\right) \\
& -v e^{h i n+\frac{q i n \delta_{2}}{K}} H\left(-\delta_{2}+\delta_{3}-p K-q i K^{\prime}\right) H\left(\zeta+\sigma-\delta_{2}-\delta_{3}\right) \\
& +e^{\left(h^{\prime}+h^{\prime \prime}\right) i \pi+\frac{q i \pi}{K}\left(\delta_{2}+\delta_{3}\right)} H\left(-\delta_{2}-\delta_{3}-p K-q i K^{\prime}\right) H\left(\zeta+\sigma-\delta_{2}+\delta_{3}\right) \text {. }
\end{aligned}
$$


270 Clebsch, ebene Curven, deren Coord. elliptische Funct. eines Parameters sind.

Nun ist

$$
\begin{aligned}
& H\left(-\delta_{2}-\delta_{3}-p K-q i K^{\prime}\right)=(-1)^{(p+1)(q+1)} e^{-\frac{\pi q i}{K}\left(\delta_{2}+\delta_{3}\right)} \cdot H\left(\delta_{2}+\delta_{3}-p K-q i K^{\prime}\right), \\
& H\left(-\delta_{2}+\delta_{3}-p K-q i K^{\prime}\right)=(-1)^{(p+1)(q+1)} e^{-\frac{\pi q i}{K}\left(\delta_{2}-\delta_{3}\right)} \cdot H\left(\delta_{2}-\delta_{3}-p K-q i K^{\prime}\right) .
\end{aligned}
$$

An Stelle der obigen Gleichung kann man daher auch folgende setzen:

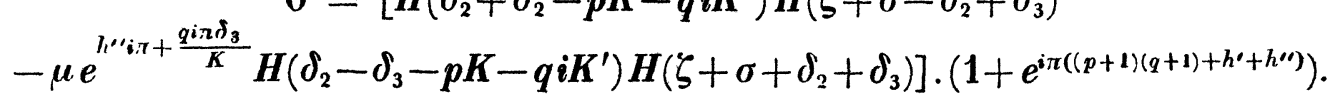

$$
0=\left[H\left(\delta_{2}+\delta_{2}-p K-q i K^{\prime}\right) H\left(\zeta+\sigma-\delta_{2}+\delta_{3}\right)\right.
$$

Der letzte Factor muss also verschwinden, d. h. man muss haben

$$
(p+1)(q+1)+h^{\prime}+h^{\prime \prime} \equiv 1 \quad(\bmod .2) \quad \text { q. e. d. }
$$

Bezeichnen wir nun eine Doppeltangente durch $\left(p, q ; h^{\prime}, h^{\prime \prime}\right)$ so sind die vier Paare von Doppeltangenten folgende:

$$
\begin{array}{llll}
(0,0 ; 0,0), & (0,0 ; 1,1) ; & (1,0 ; 1,0), & (1,0 ; 0,1) ; \\
(0,1 ; 1,0), & (0,1 ; 0,1) ; & (1,1 ; 1,0), & (1,1 ; 0,1) .
\end{array}
$$

Da die entsprechenden Zahlen zweier Paare addirt stets eine gerade Zahl geben, so folgt der Satz:

Die Berihrungspunkte je zweier Paare liegen auf einem Kegelschnitt, so dass es sechs Kegelschnitte dieser Art giebt, und sämmtliche Berïhrungspunkte dreimal auf zwei Kegelschnitten liegen.

Jede durch die Berührungspunkte zweier Paare gelegte Curve dritter Ordnung schneidet die Curve in vier Punkten, welche mit den Doppelpunkten in einem Kegelschnitt liegen, und sämmtliche Berihrungspunkte sind die Grundpunkte eines Büschels von Curven vierter Ordnung.

Giessen, den 28. October 1864. 